\title{
Quantitative analysis of protein-protein interactions governing TASK-1/TASK-3 intracellular transport
}

\author{
Dissertation \\ zur Erlangung des mathematisch-naturwissenschaftlichen Doktorgrades \\ "Doctor rerum naturalium" \\ der Georg-August-Universität Göttingen \\ im Promotionsprogramm \\ der Georg-August-University School of Science (GAUSS) \\ vorgelegt von \\ Markus Kilisch \\ aus Osterode
}

Göttingen, 2016 
Betreuungsausschuss:

1. Betreuer Prof. Dr. Ulf Diederichsen, Institut für Organische und Biomolekulare Chemie, Fakultät für Chemie

2. Betreuer Prof. Dr. Blanche Schwappach, Institut für Molekularbiologie, Universitätsmedizin Göttingen

Mitglieder der Prüfungskommission:

Referent: $\quad$ Prof. Dr. Ulf Diederichsen, Institut für Organische und Biomolekulare Chemie, Fakultät für Chemie

Korreferentin: $\quad$ Prof. Dr. Blanche Schwappach, Institut für Molekularbiologie, Universitätsmedizin Göttingen

Weitere Mitglieder der Prüfungskommission:

Prof. Dr. Peter Rehling, Institut für Zellbiochemie, Universitätsmedizin Göttingen

Prof. Dr. Claudia Höbartner, Institut für Organische und Biomolekulare Chemie, Fakultät für Chemie

Prof. Dr. Kai Tittmann, Abteilung Bioanalytik, Albrecht-von-Haller-Institut der Pflanzenwissenschaften

Prof. Dr. Michael Meinecke, Pro Futura Research Group, European Neuroscience Institute Göttingen

Tag der mündlichen Prüfung: 


\section{Acknowledgements}

I wish to express my sincere gratitude to...

... my supervisor, Blanche Schwappach, for having given me the opportunity to work on this project, for continuous scientific council, guidance, encouragement and her endless patience extended during my time in the lab

... my supervisor in the chemistry department, Prof. Dr. Ulf Diederichsen, who agreed to be my first supervisor bridging the cleft between chemistry and medicine.

... Dr. Daniela Bertinetti, who introduced me to fluorescence polarization and extended a hearty welcome to the Department of Biochemistry in Kassel, for her patience, continous scientific discussions and for letting me use their facilities without reservations.

... my colleague and friend, Dr. Eric Arakel, for the various science related and non-science related discussion, for planning and executing experiments, for his guidance and assistance during my PhD.

... the morons (friends), Jhoncito and Javi (a.k.a. marica), without whom work in the lab wouldn't have been that much fun, for handling Isabella, the SPR machine, in my absence.

... Anne, Eric, Olga, Fabio, Jhon, Javi and all former and recent members, I may have forgotten to mention here, for reading my thesis (special thanks to Anne), for extending a helpful hand performing the various experiments during my $\mathrm{PhD}$, for constant scientific council and providing a stimulating and encouraging working atmosphere.

... Jimena, the most important person in my life, who supported me during the time I was working on the paper and the time I spend isolated at home writing 
my thesis, who encouraged me to not give up halfway, for stopping me from going insane formatting the thesis and making the figures and for her endless patience and love during times of extreme stress.

... my sister, Sarah, who had to listen to all the scientific gibberish, for always being there for me.

... my parents, who supported me throughout my studies, for always being there for me and for their endless patience listening to me talking about work and who encouraged me to make everything a little more colorful. 


\section{Table of contents}

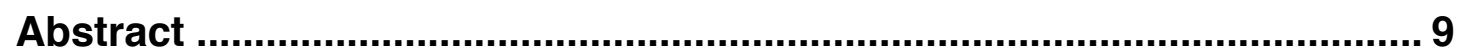

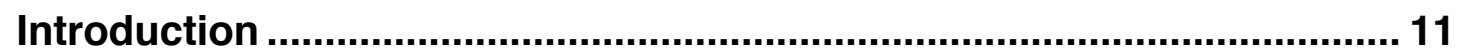

The role of protein-protein interactions in membrane protein transport along the

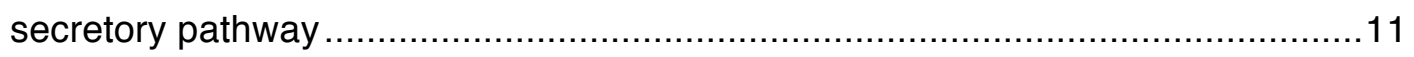

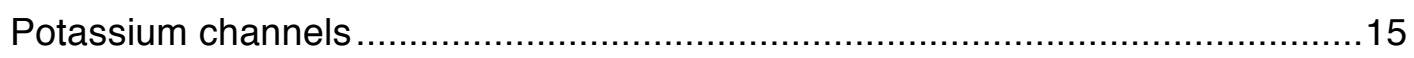

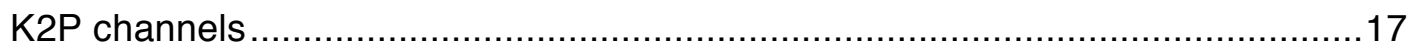

$\mathrm{K} 2 \mathrm{P}$ subunits assemble into homo- and heterodimeric $\mathrm{K}^{+}$-channels ...................18

Protein-protein interactions involved in the regulation and localization of K2P

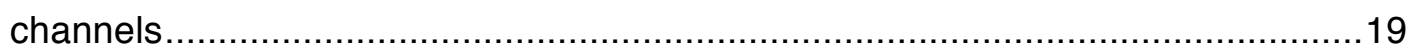

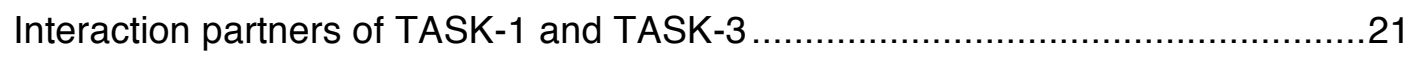

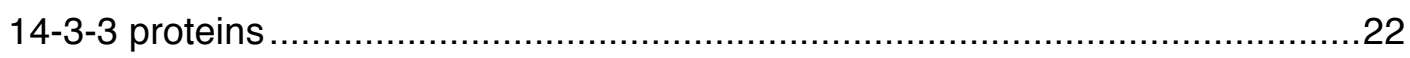

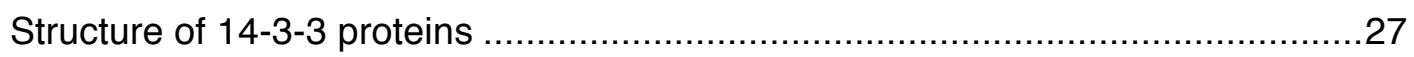

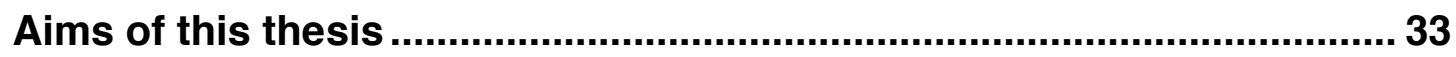

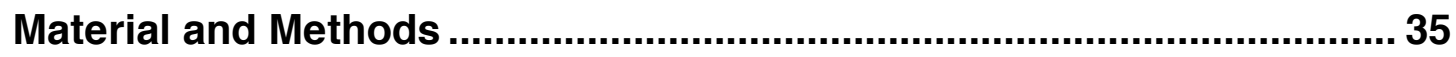

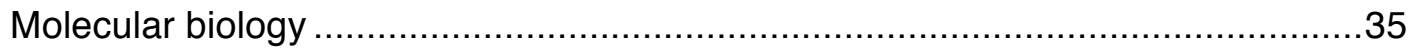

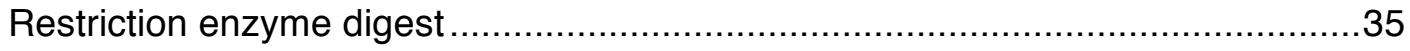

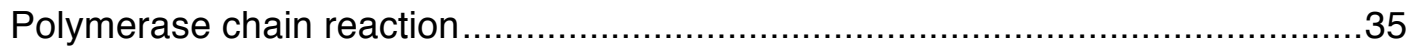

Cloning with phosphorylated oligonucleotides .....................................................

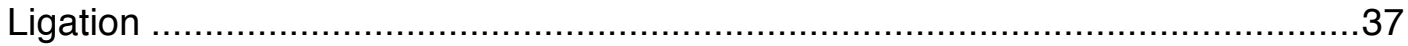

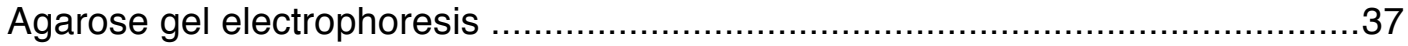

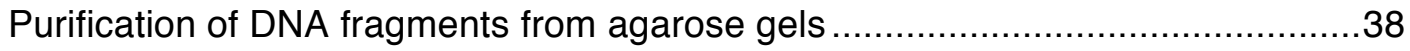

Transformation of bacterial cells by electroporation........................................38

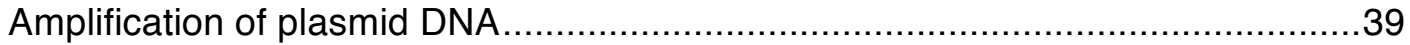

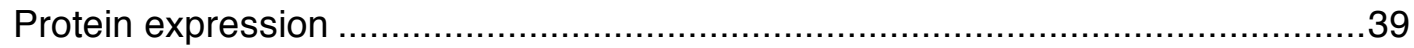

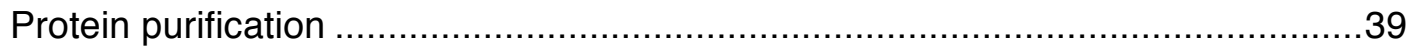

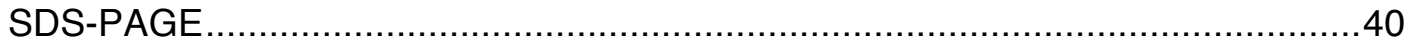

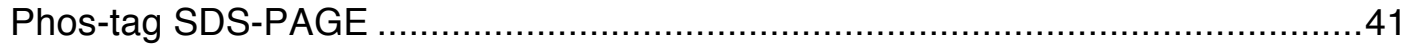

Electro blotting and Western blot detection.................................................... 41

Coomassie staining of protein gels ............................................................... 42

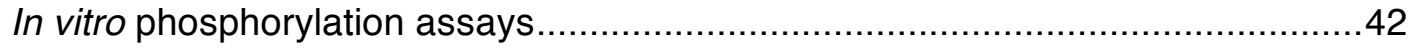

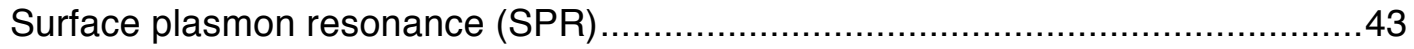

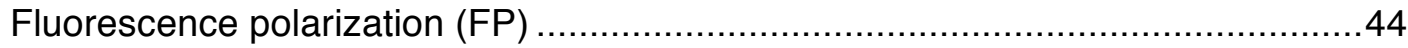




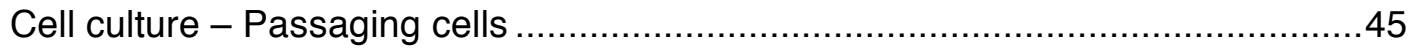

Cell culture - Transient transfection........................................................... 46

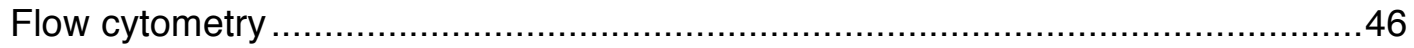

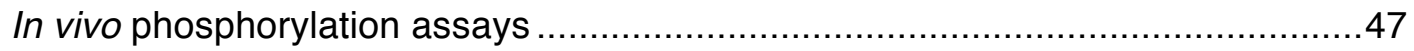

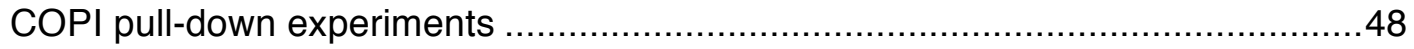

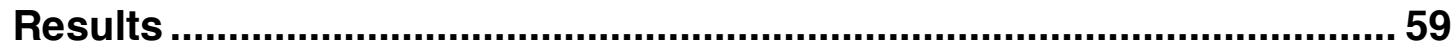

Quantitative characterization of 14-3-3 binding to TASK-derived C-terminal peptides 59

14-3-3 proteins bind the TASK-3 C-terminus phosphorylation dependent and with high affinity 60

Introducing a K369A mutation into the TASK3 C-terminus does not disrupt 14-3-3 binding 63

Deletion of V374 does not abolish 14-3-3 binding 65

Modulation of 14-3-3 binding by differential phosphorylation of the client protein .68 Binding affinities of 14-3-3 proteins for the TASK-1 WT C-terminus are significantly lower than for the TASK-3 WT C-terminus.......................................................69

Truncation of the TASK-1 C-terminus disrupts 14-3-3 binding ..........................70

Phosphorylation of S392 inhibits 14-3-3 binding ............................................ 72

Correlation of 14-3-3 binding parameters by Surface Plasmon Resonance (SPR)78

TASK-1 and TASK-3 C-termini are phosphorylated by PKA in vitro.....................84

Deletion of the distal Valine V374 in TASK-3 affects PKA phosphorylation ..........86

Cell surface expression of different TASK-derived reporter proteins .....................91

Deletion of V374 decreases the efficiency by which PKA phosphorylates the TASK-3 C-terminus in vivo.

Cell surface expression of TASK-1-derived reporter proteins reveals a potential regulatory role of $\mathrm{S} 392$

Transient phosphorylation of S392 reduces the relative cell surface expression of TASK-1-derived reporter proteins .............................................................. 102

Phosphorylation of TASK-1 and TASK-3 C-termini impairs COPI binding...........105

Discussion. 109

Small sequence differences in TASK-3 and TASK-1 cause biological relevant

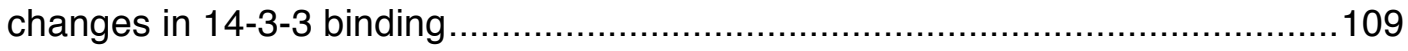

Phosphorylation of S392 in TASK-1 impairs COPI and 14-3-3 binding ..............112 
Quantitative evaluation of two distinct mutations of TASK-1 and TASK-3 thought to abolish 14-3-3 binding 115

Differences in cell surface expression of TASK-1 and TASK-3 derived reporterproteins reflect differences in 14-3-3 and COPI binding 117

Truncated TASK C-termini are less efficiently phosphorylated in vivo. 119

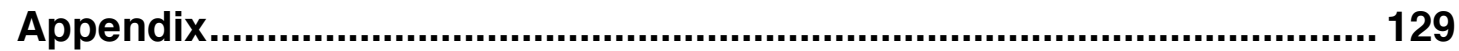

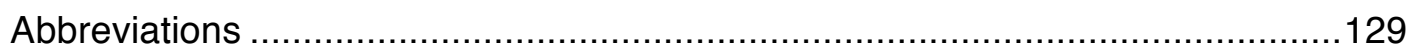

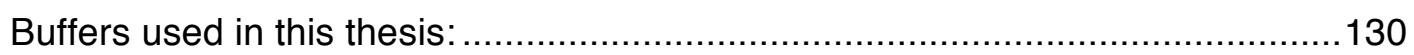

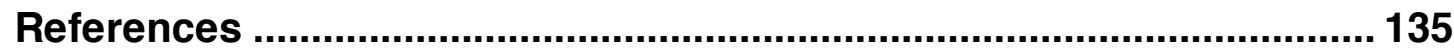




\section{Abstract}

The transport of the K+-channels TASK-1 and TASK-3 to the cell surface is regulated by protein-protein interactions with either the COPI vesicle coat, or members of the phosphoadaptor protein family 14-3-3. Interactions are mediated via a trafficking control region present at the distal C-terminus of either $\mathrm{K}+$-channel. This trafficking control region comprises a polybasic ER retention and retrieval motif and an adjacent mode III 14-3-3 binding motif. Phosphorylation of a conserved serine residue, as part of the mode III 14-3-3 binding motif, is followed by the recruitment of 14-3-3, thereby releasing the channel from ER retention by sterically preventing the COPI vesicle coat from binding to the overlapping ER retention and retrieval motif. Following phosphorylation and 14-3-3 binding, the channel is transported forward to the cell surface. In this thesis I determined the binding parameters of all seven human 14-3-3 isoforms to the trafficking control regions of TASK-1 and TASK3. Furthermore, I investigated the direct effect of phosphorylation of the TASK1 and TASK-3 C-terminus on COPI binding. I observed distinctly different binding parameters between individual 14-3-3 isoforms and different channel C-termini demonstrating that 14-3-3 isoforms bind the same substrate in an isoform specific manner. Surprisingly, the binding affinities determined for TASK-1 were approximately two orders of magnitude lower than the binding affinities determined for TASK-3. I explain these differences by small, but physiologically relevant, amino acid sequence differences within the trafficking control regions of TASK-1 and TASK-3. While TASK-3 presents a second lysine residue that allows for high affinity binding of 14-3-3 proteins to this trafficking control region, TASK-1 presents a second serine residue that upon phosphorylation inhibits 14-3-3 binding. I further correlate my in vitro observations with reporter protein assays performed in vivo (COS7), assessing the relative cell surface expression of TASK-derived reporter proteins. My findings indicate that the control of TASK-1 protein trafficking is highly dynamic, modulated by COPI, 14-3-3, kinases and phosphatases. Binding experiments performed with the yeast COPI vesicle coat and phosphorylated or unphosphorylated constructs comprising the distal C- 
terminus of TASK-1 and TASK-3 (the last 15 amino acids) demonstrate that the phosphorylation of these trafficking control regions is sufficient to interfere with COPI binding, in absence of 14-3-3. In summary, my findings contribute substantially to the quantitative understanding of events governing the intracellular transport of TASK-1 and TASK-3. 


\section{Introduction}

The role of protein-protein interactions in membrane protein transport along the secretory pathway

Different cell types, such as neurons and cardiac myocytes, are required to perform highly specific functions. To achieve a directed and coordinated response to external stimuli with its neighboring cells, each cell needs to be aware of its surroundings and sense changes in its environment. Several classes of surface membrane proteins enable this sensing, e.g. receptor proteins, ion-channels, transporters and structural proteins (1). To maintain the function and physiology of such cell types the cell surface expression of distinct membrane proteins is required to be tightly regulated, whereas deviation and disturbance of this dynamic equilibrium can lead to severe phenotypes, such as arrhythmias and neurodegenerative diseases (2). The cell surface expression of each surface membrane protein is regulated by interactions with other proteins, e.g. vesicular coat proteins, which enable the cell to tailor specific sorting steps to the needs of an individual membrane protein while facilitating the anterograde or retrograde transport of various proteins along the secretory pathway (3).

In general, protein-protein interactions can affect the biogenesis of membrane proteins in different ways. Interactions with chaperone-like proteins allow for an enhanced folding of the protein into its native state, or can have a stabilizing effect on intrinsically unstable macromolecules (3-5). Interactions with regulatory and inhibitory subunits can regulate the activity of membrane proteins, e.g. $\mathrm{K}^{+}$-channels (6). Furthermore, interactions with vesicular coat proteins can affect the localization of membrane proteins and thereby modulate the cell surface expression of a membrane protein and consequently alter the biophysical properties of a distinct cell-type (7).

Interactions between cargo and vesicle coat or trafficking adaptor proteins are often mediated by short peptide stretches, commonly referred to as trafficking 


\section{Introduction}

control, or sorting motifs. For example, the retrieval of membrane proteins to early compartments of the secretory pathway is mediated by di- or tribasic amino acid motifs that are recognized by the COPI vesicle coat. These motifs have been classified as canonical retention and retrieval motifs RKXX (8), KKXX (9), RXR (10) and non-canonical retention and retrieval motifs, e.g. KRR (11). Another type of trafficking motif was found to facilitate the internalization of membrane proteins from the cell surface via interactions with components of the clathrin vesicle coat. The two most commonly instances of this motif can be classified as $\mathrm{YXX \Phi}$ and the di-leucine containing motif [DE]XXXL[LI], with $X$ representing any amino acid and $\Phi$ representing a hydrophobic amino acid (12). Some protein-protein interactions require the previous posttranslational modification of the client protein, e.g. recognition of clients by 14-3-3 proteins. Thus interactions can be modulated by kinases and phosphatases. Interactions with 14-3-3 proteins are mediated via discrete peptide motifs, such as mode I (RSX(pS/pT)XP), mode II (RXФX(pS/pT)XP) (13) and mode III $\left(\operatorname{RXSX}_{(1-2)}-\mathrm{COOH}\right)(14,15)$ 14-3-3 binding motifs. The cell surface expression of multiple membrane proteins was found to depend on the mutually exclusive binding of either COPI, or 14-3-3, e.g. to the cargo proteins Kir6.2, TASK-1, TASK-3, ADAM22 or human $a 4$ (1, 3, 6, 11, 16-19), further emphasizing the importance of 14-3-3 and COPI in membrane protein trafficking. Figure 1 illustrates the complexity of the underlying mechanism that targets the membrane protein ADAM22 to the cell surface. This example is particularly informative since the phosphorylation-dependent binding of 143-3, similar to TASK-1 and TASK-3, promotes the expression of ADAM22 at the cell surface. At the same time, Golgi passage of ADAM22 can be monitored based on its maturation (cleavage) in this compartment $(20,21)$.

The presence of $\mathrm{K}^{+}$-selective ion channels at the cell surface can be studied with high resolution $(3,22)$. However, detailed insight into the different mechanisms that regulate the cell surface expression of different $\mathrm{K}^{+}$-channels is lacking. I am particularly interested in the mechanism modulating the cell surface expression of two-pore-domain acid-sensitive $\mathrm{K}^{+}$-channels (TASK). 
The cell surface expression of TASK-1 and TASK-3 has previously been found to depend on the mutually exclusive binding of either COPI, vesicular coat proteins that retrieve the protein in early compartments of the secretory pathway, or 14-3-3, a phosphoadaptor protein that binds in a phosphorylation dependent manner to a trafficking control region located at the distal Cterminus of both channels $(11,17,18)$. This mechanism is thought to operate based on the notion that COPI binding collects and retrieves the protein to the ER until the client protein is phosphorylated by PKA (23) and subsequently associates with 14-3-3. Interactions with 14-3-3 are mediated via a mode III 14-3-3 binding motif $(14,15)$ and $14-3-3$ binding is thought to sterically prevent COPI from binding to an adjacent ER retention and retrieval motif, thus facilitating the forward transport of the channel to the cell surface. This thesis aims to quantitatively evaluate binding events thought to regulate the cell surface expression of TASK-1 and TASK-3. Thereby, I would like to contribute to elucidating the physiological role, structure and function of various $\mathrm{K}^{+}$-channels and the importance of 14-3-3 in various cellular processes. In a detailed analysis of the relationship between 14-3-3 and binding properties to the trafficking control region of TASK channels I endeavor to gain insight into the complexity of 14-3-3 isoform-specific binding. 


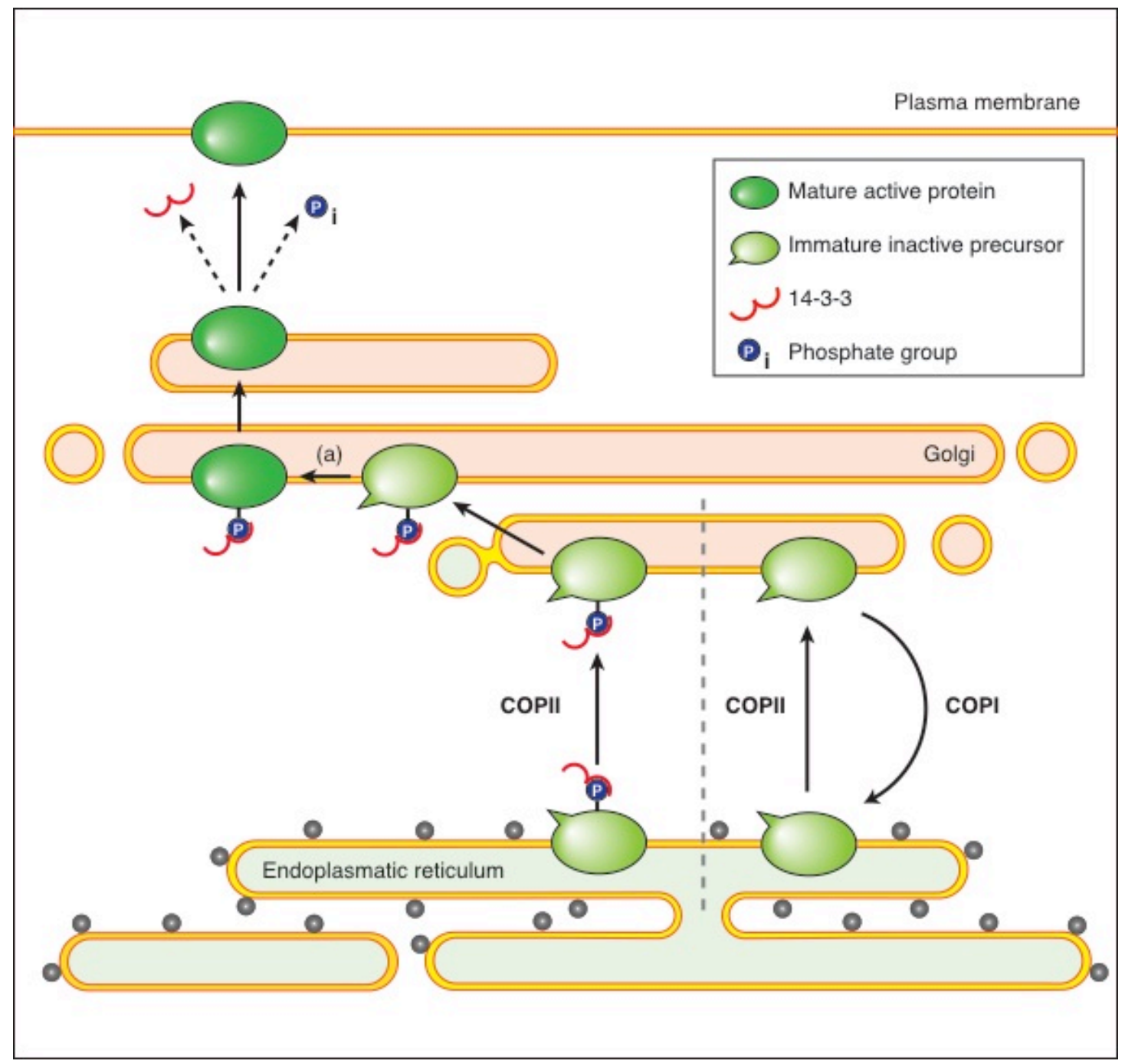

Figure 1: Protein-protein interactions involved in the cell surface targeting of membrane proteins. The cell surface targeting of the membrane protein ADAM22 involves multiple protein-protein interactions that are employed to regulate the transport and the activation of the protein on its way to the cell surface. Following synthesis and insertion into the membrane of the endoplasmatic reticulum (ER) the immature and inactive precursor protein of ADAM22 is transported forward to the Golgi. Two RXR-type ER retention and retrieval motifs have been identified in ADAM22 that when exposed mediated interactions with the COPI vesicle coat, returning the protein to early compartments of the secretory pathway. Both retention and retrieval motifs partially overlap with adjacent 14-3-3 binding motifs. Phosphorylation of these 14-3-3 binding motifs results in the recruitment of 14-3-3 proteins (21). This 14-3-3 binding is thought to sterically prevent COPI from binding, thus releasing the protein from ER retention and allowing for progression of the immature precursor through different compartments of the Golgi. Within the Golgi, the 
immature and inactive precursor protein is proteolytically processed (a). This processing converts the immature and inactive precursor into its active form (20), making the pre- and post-Golgi population of ADAM22 readily distinguishable (20). Upon exit from the Golgi, the mature and active protein dissociates from 14-3-3 and is subsequently dephosphorylated (21). This example emphasizes the role of COPI and 14-3-3 in membrane protein transport. The basic layout was adapted from Smith et al., 2011 (1).

\section{Potassium channels}

$\mathrm{K}^{+}$-channels are ubiquitous and can be found in diverse cellular membranes of the animal and plant kingdom. $\mathrm{K}^{+}$-channels represent the most abundant ionchannel family in vertebrates with more than 78 genes encoding for poreforming subunits (24). In general, $\mathrm{K}^{+}$-channels regulate the flux of $\mathrm{K}^{+}$-ions across the plasma membrane, setting the membrane resting potential and maintaining the electrical excitability of cell-types such as neurons and cardiac myocytes $(2,6,24,25)$. The $\mathrm{K}^{+}$-channel superfamily can be divided into different structural subclasses namely inwardly-rectifying $\mathrm{K}^{+}$-channels (Kir), voltage gated $\mathrm{K}^{+}$-channels $(\mathrm{Kv})$ and two-pore domain $\mathrm{K}^{+}$-channels (K2P). Whereas $\mathrm{Kv}$ and $\mathrm{K} 2 \mathrm{P}$ channels are involved in the repolarization of the membrane following an action potential, Kir channels stabilize the membrane potential close to the $\mathrm{K}^{+}$equilibrium potential (26).

Each subfamily possesses its very own distinct membrane topology: Kir channels comprise one pore-forming domain (P-domain) and two transmembrane segments (1P-4M); Kv channels contain in addition to one poreforming domain six trans-membrane segments, of which the fourth transmembrane segment functions as a voltage sensor (1P-6M). In contrast to Kir and $\mathrm{Kv}$ channels, K2P channels are composed of two pore-forming domains and four trans-membrane segments (2P-4TM) (26). Although, each subfamily displays a large structural diversity, a 20 amino acid long stretch within the pore-forming domain is remarkably well conserved across different $\mathrm{K}^{+}$- 


\section{Introduction}

channels. This sequence contains a T-X-G-X-G motif, which was initially employed to screen the mammalian genome for $\mathrm{K}^{+}$-channel subunits and is also known as the ' $\mathrm{K}^{+}$-channel signature sequence' $(27,28)$. A fully functional $\mathrm{K}^{+}$-channel comprises four $\mathrm{P}$-domains, which form a $\mathrm{K}^{+}$-selective pathway across the membrane. Consequently, Kir and $\mathrm{Kv}$ channels assemble into tetramers with each channel subunit contributing one P-domain and K2P channels, containing two P-domains, accordingly assemble into dimers (29, 30). The membrane topology of each $\mathrm{K}+$-channel is depicted in Figure 2.

A

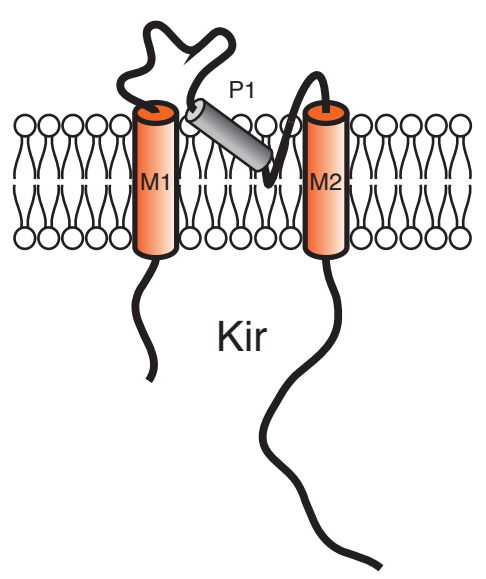

B

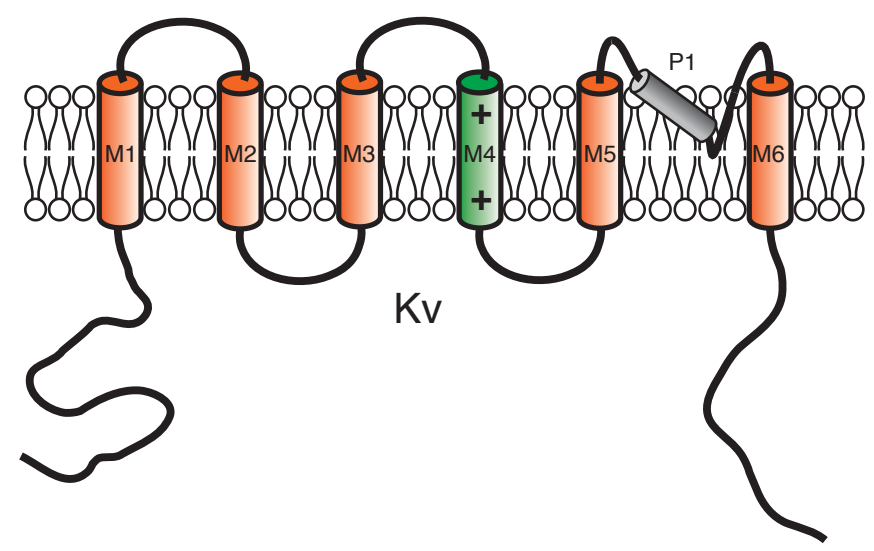

C

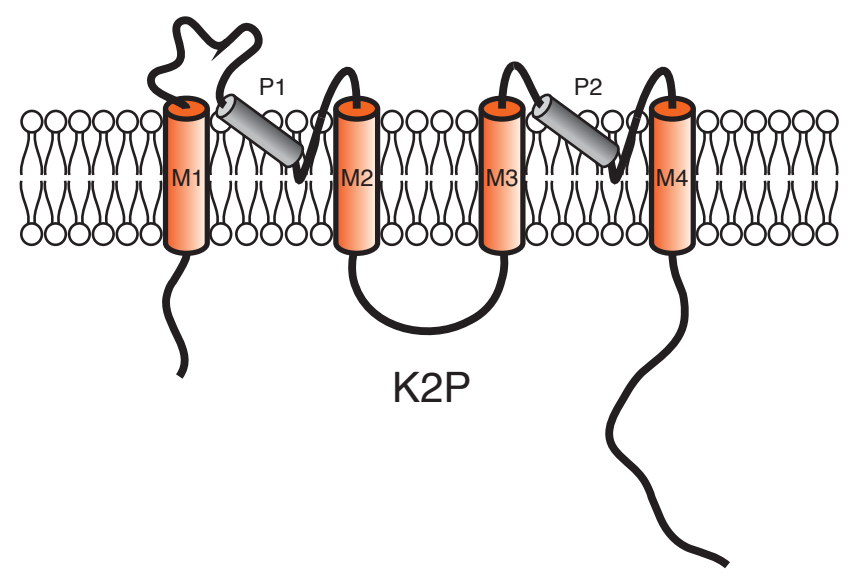

Figure 2: Membrane topology of different $\mathrm{K}+$-channels. A: The predicted membrane topology of inward-rectifying $\mathrm{K}^{+}$-channels (Kir). Two trans-membrane segments (M1 and $\mathrm{M} 2$ ) and one pore-forming domain (P1) are shown. B: The membrane topology of voltage gated $\mathrm{K}^{+}$-channels $(\mathrm{Kv})$. Six transmembrane segments (M1 to M6) and one pore-forming domain are shown. The voltage sensor is depicted in green (M4). C: A 
simplified scheme of the membrane topology of two pore-domain potassium channels (K2P). Four transmembrane segments (M1 to M4) and two pore-forming subunits are shown. The $\mathrm{N}$ - and $\mathrm{C}$-termini of each $\mathrm{K}^{+}$-channel subunit are located within the cytosol. A functional channel requires four pore-forming domains. Thus functional Kir and $\mathrm{Kv}$ channels consist of four subunits and K2P channels of two channel forming subunits (adapted from O'Connell et al., 2002 (26)).

\section{K2P channels}

Currently 15 genes encoding for pore-forming subunits of K2P channels within the mammalian genome are known. Based on sequence conservation, functional properties and localization, these channels were divided into six major subgroups. The first subgroup is classified as weakly inward rectifying $\mathrm{K} 2 \mathrm{P}$ channels (TWIK), according to the properties of the first mammalian K2P cloned by Lesage et al. in 1996. This group comprises TWIK-1 (K2P 1.1; KCNK1 (31)), TWIK-2 (K2P 6.1; KCNK6 (32)) and TWIK-3 (K2P 7.1; KCNK7 (33)). The second subgroup is comprised of channels, which are lipid and mechanosensitive (TREK). K2P channels classified as such are TREK-1 (K2P 2.1; KCNK2 (34)), TREK-2 (K2P 10.1; KCNK10 (35)) and TRAAK (K2P 4.1; KCNK4 (36)). The third subgroup contains channels that are highly sensitive to changes in extracellular acidification and were accordingly named two poredomain acid-sensitive K2P channels (TASK). This subgroup of K2P channels contains three members: TASK-1 (K2P 3.1; KCNK3 (37)), TASK-3 (K2P 9.1; KCNK9 (38, 39)) and TASK-5 (K2P 15.1; KCNK15 (40-42)). Although expression of TASK-5 in oocytes or mammalian cells yielded non-functional, 'silent' K2P channels, structural similarities to TASK-1 and TASK-3 classified this channel as acid-sensitive. The fourth subgroup of potassium channels is inactivated upon acidification of the extracellular matrix and exhibits high $\mathrm{pH}$ sensitivity in the alkaline range. Accordingly, this subgroup of potassium channels was classified as two pore-domain alkaline-pH sensitive K2P channels (TALK), comprising TASK-2 (K2P 5.1; KCNK5 (43)), TALK-1 (K2P 16.1; KCNK16 (44)) and TALK-2 (K2P 17.1; $\operatorname{KCNK17~}(44,45))$. Although 


\section{Introduction}

TASK-2 was initially grouped with acid-sensitive K2P channels, low sequences similarity with TASK-1 and the inactivation of the channel upon extracellular acidification attributed this channel to the TALK subgroup. The fifth subgroup of $\mathrm{K} 2 \mathrm{P}$ channels was classified as tandem pore domain halothane inhibited $\mathrm{K}^{+}$-channels comprising THIK-1 (K2P 13.1; KCNK13 (46)) and THIK-2 (K2P 12.1; KCNK12 (46)). The last mammalian K2P channel discovered was named according to its predominant expression in sensory neurons TWIK-related spinal cord $\mathrm{K}^{+}$-channel (TRESK). This subgroup comprises only one member TRESK (K2P 18.1; KCNK18 (47)).

\section{K2P subunits assemble into homo- and heterodimeric $\mathrm{K}^{+}$-channels}

Two K2P channel subunits are required to constitute a functional $\mathrm{K}^{+}$-selective ion-channel. Each subunit comprises two pore-forming domains (P-domain) and four transmembrane segments (TM), with the first P-domain located between TM1 and TM2 and the second P-domain located between TM3 and TM4. The selectivity filter forms a $\mathrm{K}^{+}$-selective pore across the membrane and comprises four P-domains. In Figure 3 the general membrane topology of $\mathrm{K} 2 \mathrm{P}$ channels is illustrated. Current literature demonstrates that homodimeric K2P-channels exhibit unique biophysical properties when expressed at the cell surface (31-47). Based on structure predictions and similarities found to be relevant for channel regulation $\mathrm{K} 2 \mathrm{P}$ channels could potentially form heterodimeric, functional $\mathrm{K}^{+}$-channels (48).

A study by Czirj'ak \& Enyedi (2002) reported the formation of a heterodimeric $\mathrm{K}^{+}$-channel composed of TASK-1 and TASK-3 in vitro. These channels were found to partially retain properties of each monomer, which constitute the channel. Furthermore it was found that the novel (heterodimeric) channel exhibited electrophysiological properties different to homodimeric TASK-1 and TASK-3 $\mathrm{K}^{+}$-channels $(48,49)$. The formation of heterodimeric K2P channels was later found to be physiologically relevant in different cell-types, such as cerebellar granule neurons (50). In these cells TASK-1/TASK-3 heterodimeric $\mathrm{K}^{+}$-channels are responsible for a major part of the 'standing' outward current. 
Furthermore it could be shown that approximately one half of the background current detected in motoneurons is caused by TASK-1/TASK-3 heterodimers $(49,51)$. Recently Kim et al. (2009) demonstrated that the major part of the $\mathrm{pH}$-sensitive background $\mathrm{K}^{+}$current in carotid body glomerulus cells is regulated by TASK-1/TASK-3 heterodimers $(52,53)$. Besides TASK-1 and TASK-3 other K2P channels have recently been reported to form heterodimeric and functional channels. Blin et al. (2014) studied the formation of functional channels between THIK-1 and THIK-2 (54). Whereas Plant et al. (2012) described the formation of a heterodimeric and functional channel of TWIK-1 with either TASK-1 or TASK-3, followed by Hwang et al. (2014) who demonstrated that heterodimeric channels are formed between TWIK-1 and TREK-1 $(55,56)$. These reports emphasize the formation of heterodimeric $\mathrm{K} 2 \mathrm{P}$ channels as a cellular mechanism to acquire distinct properties in a cost efficient way (57).

\section{Protein-protein interactions involved in the regulation and localization of K2P channels}

A tight equilibrium between forward transport of $\mathrm{K}^{+}$-channels along the secretory pathway and endocytosis from the plasma membrane is required to maintain the electrical properties of excitable tissue, such as neurons and cardiac myocytes (58). The number of $\mathrm{K}^{+}$-channels reaching the cell surface can either be regulated on the transcriptional level (59-62) or the posttranslational level $(11,17,18,63,64)$. Interactions with accessory proteins affect the function and/or the localization of the $\mathrm{K}^{+}$-channel.

For example, a study by Sandoz et al. (2008) reported that TREK-1 and TREK-2 interact directly with the microtubule-associated protein 2 (Mtap2). Co-expression of Mtap2 with TREK-1 or TREK-2 led to an increased cell surface expression of these channels (63). These observations could later be explained by simultaneous binding of Mtap2 to TREK-1, or TREK-2 and microtubules facilitating increased forward transport of the channel to the cell surface (63). Another study by Sandoz et al. (2006) demonstrated that direct 


\section{Introduction}

interactions of TREK-1 and TREK-2 with the A-kinase anchoring protein (AKAP)-150 stimulate channel activity (65). The binding of AKAP-150 occurs via the fourth transmembrane segment of either subunit.

A

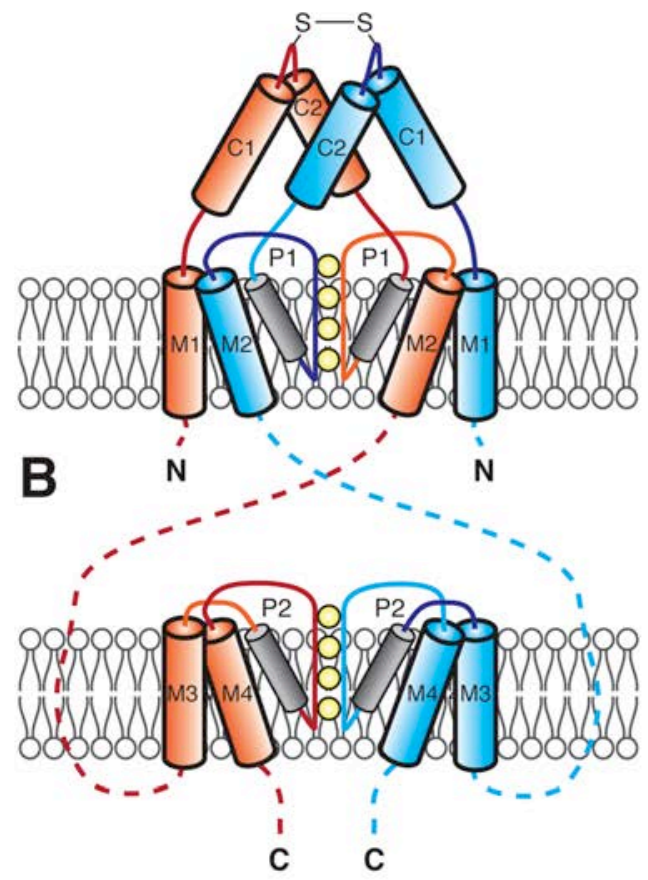

C

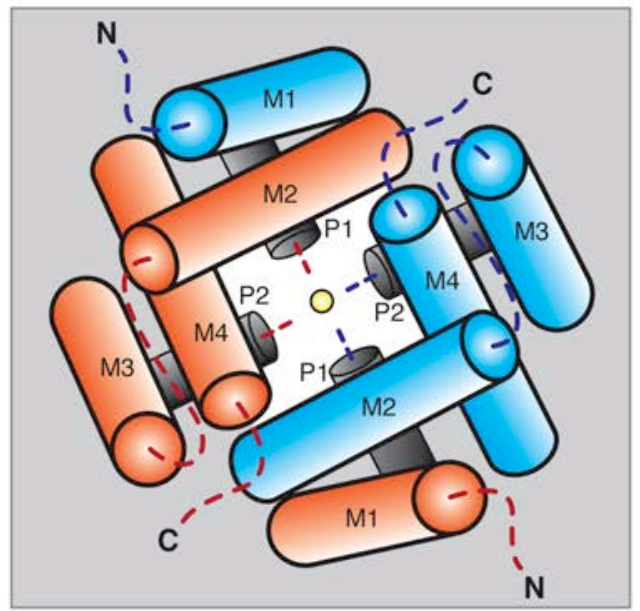

D

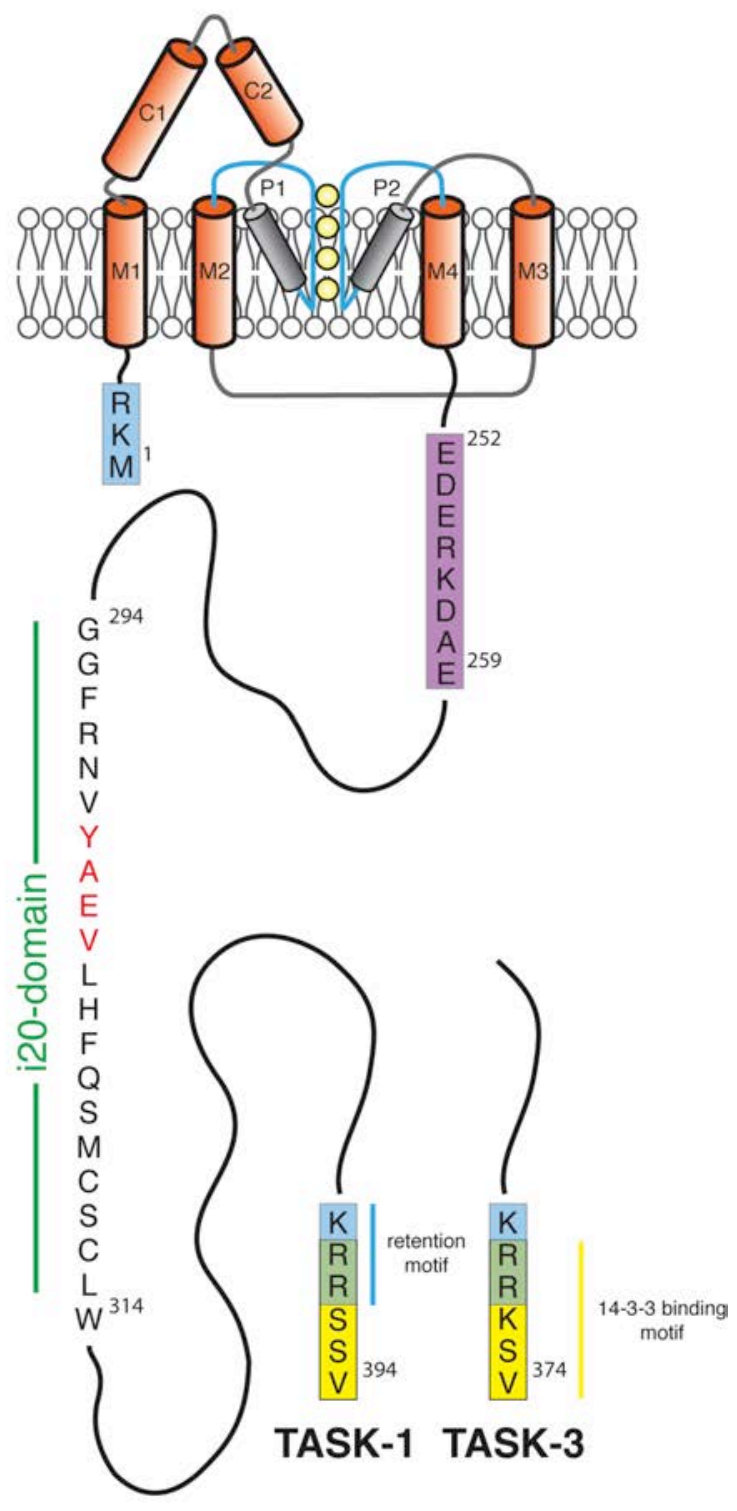

Figure 3: General membrane topology of K2P channels. The illustrated structure is based on a crystal structure reported for TWIK-1 by Miller et al., (2012) (29). A: A refined structural model of the membrane topology of K2P channels. Depicted are the $\mathrm{N}$-terminal region of $\mathrm{K} 2 \mathrm{P}$ channels including $\mathrm{M} 1, \mathrm{M} 2, \mathrm{P} 1$ and both cap-domains $(\mathrm{C} 1$, C2). Observations made for TWIK-1 suggest a covalent linkage between both pore- 
forming subunits via cysteine residues present in the loop connecting $\mathrm{C} 1$ and $\mathrm{C} 2$. TASK-1 and TASK-3 lack these cysteine residues. A crystal structure reported by Miller et al. (2012) showed that transmembrane segment M1 of subunit one (red) and M2 of subunit two (blue) forming intricate contact sites. B: The C-terminal region of K2P channels including M3, M4, P2 and the unstructured C-terminal region of each subunit (dashed line) is depicted. D: View of the channel from the cytosolic side. The general alignment of the different transmembrane segments and pore-forming domains is based on a crystal structure of TWIK-1 (29). D: Functional topology of TASK-1 and TASK-3, highlighted in blue are ER retention, or retrieval motifs; highlighted in yellow are 14-3-3 binding domains. TASK-1 possesses an additional 20 amino acid long i20-domain that binds to the cytosolic retention factor p11, which is absent in TASK-3. Highlighted in purple and in red letters are potential endocytosis motifs. The channel subunit is comprised of four trans-membrane segments (M1 M4), two pore-forming domains (P1, P2) and two cap-domains (C1, C2). The helices are not drawn to scale. The pore-forming domains are represented as smaller helices for the purpose of illustration and $\mathrm{K}^{+}$-ions are depicted as yellow spheres (adapted from Kilisch et al., 2015 (3)).

\section{Interaction partners of TASK-1 and TASK-3}

TASK-1 and TASK-3 have been found to associate with various interaction partners, which affect their activity or localization, in vivo. A report by Rajan et al. (2002) demonstrated that TASK-1, TASK-3 and TASK-5 directly interact with proteins of the phosphoadaptor protein-family 14-3-3 and that association with 14-3-3 is required to release TASK-1 and TASK-3 from ER retention (17). Channels which failed to associate with 14-3-3, were strongly retained in compartments of the early secretory pathway (17). In parallel, O'Kelly et al. (2002) demonstrated that the retention of TASK-1 is mediated by interactions of a dibasic N-terminal ER retention motif $(K R)$ with the COPI vesicle coat (18). Both reports emphasize that phosphorylation of a C-terminal serine residue is essential to mediate interactions with 14-3-3 $(17,18)$. A subsequent study by Zuzarte at al. (2009) described interactions of TASK-1 and TASK-3 with COPI as mediated via a tribasic, non-canonical ER retention and retrieval 


\section{Introduction}

motif (KRR), located at the distal C-terminus of either channel and that binding of COPI to this trafficking control motif is sufficient to retain these channels intracellularly (11). Recently, Mant et al. (2011) demonstrated that proteinkinase $A(P K A)$ was capable of phosphorylating the conserved serine residue present at the distal C-terminus of TASK-1 and TASK-3, in vitro (23). The conserved serine residue is part of a mode III 14-3-3 binding motif and phosphorylation leads to the recruitment of 14-3-3 proteins and subsequent release of TASK-1 and TASK-3 from ER retrieval by the COPI vesicle coat. Mant et al. (2011) further demonstrated that other kinases, which recognize a similar phosphorylation consensus site, failed to phosphorylate different TASK C-termini in vitro, indicating a potential involvement of PKA in the modulation of TASK-trafficking in vivo $(1,23)$. A study by Renigunta et al. (2006) reported that TASK-1 interacts with another cytosolic adaptor protein, p11 (A100S10). Interactions with p11 occur via a 20 amino acid long C-terminal region (i20domain) upstream of the previously described trafficking control region and lead to a collective retrieval of the adaptor protein and the channel to intracellular compartments (66). A subsequent study by Renigunta et al. (2014) reported interactions of TASK-1 with the endosomal SNARE protein Syntaxin-8. Association with Syntaxin-8 is thought to allow for cooperative endocytosis of the SNARE protein and TASK-1 (67). Both reports demonstrate that the cell surface expression of TASK-1 is tightly regulated and that multiple factors alter the localization of TASK-1 in vivo $(66,67)$. In Figure 3 the functional topology of TASK-1 and TASK-3 is illustrated, highlighting residues involved in protein-protein interactions, which influence the subcellular localization of TASK channels.

\section{4-3-3 proteins}

The ubiquitous family of phospho-adaptor proteins, called 14-3-3, was initially described by Moore and Perez (1967) and named accordingly by its position on two-dimensional DEAE chromatography and subsequent starch gel electrophoresis (68). The most intriguing observation made was that 14-3-3 proteins were highly abundant in the mammalian brain, with approximately $1 \%$ 
of the total soluble protein. Subsequent studies emphasized the uniquely important role of 14-3-3 proteins in brain and neuronal function (69-71). The understanding of 14-3-3 proteins as a heterogeneous family of proteins with a molecular mass of approximately $30 \mathrm{kDa}$ that form homo- and heterodimers (Figure 4), which persists up until today, was gained only 20 years after their discovery (72). In 1987, Ichimura et al. reported that 14-3-3 proteins play a key-role in the activation of tyrosine and tryptophan hydroxylase in the presence of calcium and calmodulin-dependent protein kinase II (CaMKII) (69, 73, 74). Following these observations, systematic studies identified and cloned representative members of the 14-3-3-protein family from bovine brain $(75,76)$. According to their elution position after reverse-phase highperformance liquid chromatography (HPLC) Greek letters ( $a, \beta, \gamma, \delta, \varepsilon, \zeta$, and $\eta)$ were assigned to the different 14-3-3 proteins (75). Two additional 14-3-3 isoforms were later found in $\mathrm{T}$ - and epithelial cells and accordingly termed 14-

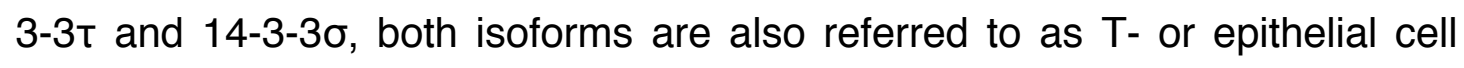
markers, $\tau$ and $\sigma(77,78)$. Subsequent studies demonstrated that 14-3-3 proteins could be found in all eukaryotic organisms, i.e. mammals, insects, plants and yeast and that 14-3-3 proteins are ubiquitously expressed in different tissues $(79,80)$. Furthermore 14-3-3 proteins were found to be involved in various cellular processes, such as modulation of protein kinase $\mathrm{C}$ (PKC) activity (81, 82), activation of calcium-dependent exocytosis in permeabilized adrenal chromafin cells $(83,84)$, stimulation of exoenzyme $S$ of Pseudomonas aeroginose (85), association with the G-box DNA-protein complex in maize and Arabidopsis thaliana (86) and function as binding partner of the phytotoxin fusicoccin (87). Furthermore it was shown that 14-33 proteins are involved in cell-cycle-control of the DNA-damage checkpoint in yeast (88), that 14-3-3 proteins form a complex with the protein kinases c-Bcr and Bcr-Abl (89) and that 14-3-3 proteins associate with the mammalian cdc25 phosphatase (90), by which the entry into the cell cycle is regulated (91). A study by Muslin et al. (1996) demonstrated that interactions between 14-3-3s and their client proteins are mediated via phosphorylated residues and further proposed that the amino acid sequence RSXPSXP represents the 


\section{Introduction}

structural basis for specific and high affinity 14-3-3 binding (92). In parallel Zha et al. reported that binding of 14-3-3 to the pre-apoptotic protein BAD prevented apoptosis, interfering with the formation of a complex between $\mathrm{Bcl}-$ 2 , or $B c l-X_{L}$ and BAD. They further demonstrated that binding of $14-3-3$ is directly dependent on phosphorylation of two potential phosphorylation sites

A

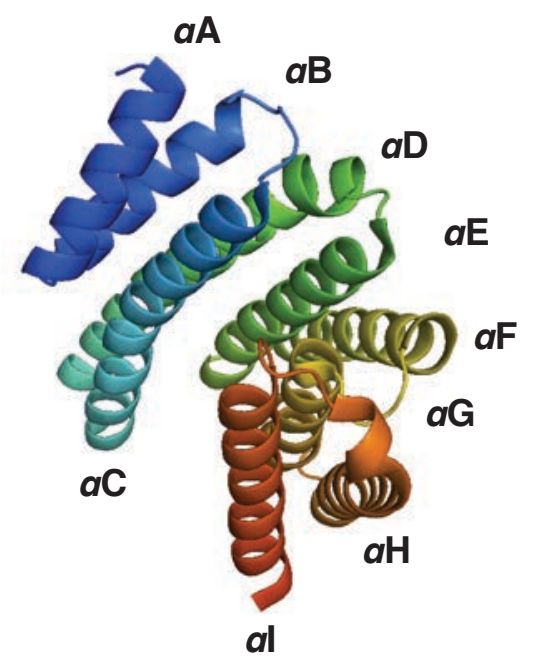

B

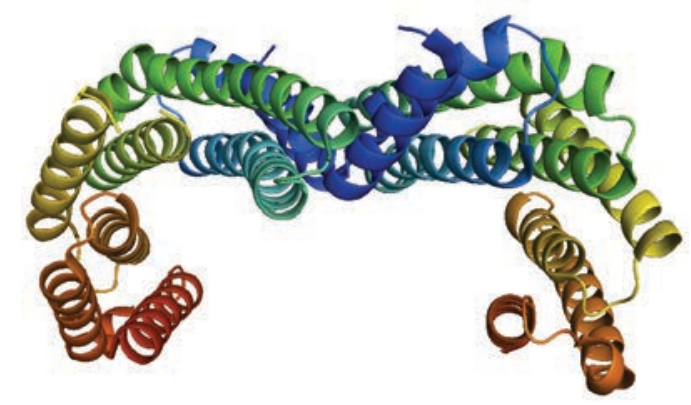

D

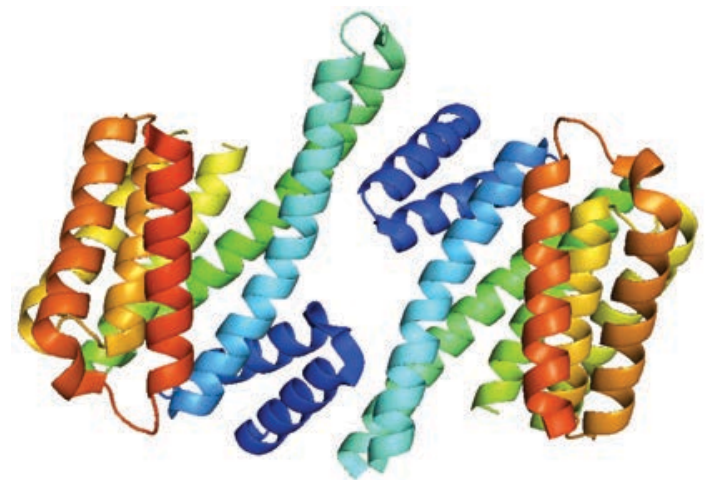

Figure 4: Fundamental structure of $14-3-3$ proteins. A: The common structure of a 14-3-3 monomer composed of nine $a$-helices. The helices are labeled from the $\mathrm{N}$ terminal end to the C-terminal end with aA (blue) to al (red). B: Two 14-3-3 monomers constitute a 14-3-3 dimer. The representative crystal structure for a 14-3-3 homodimer of 14-3-3乙 (93) is illustrated. C: The same 14-3-3 dimer as in B, rotated by $90^{\circ}$ along the horizontal axis (top view). D: The same crystal structure of 14-3-3ろ as in $\mathrm{B}$ and $\mathrm{C}$, rotated by $180^{\circ}$ along the horizontal axis (bottom view). The crystal structure of 14-3-3 $\zeta$ was obtained from the protein database Europe (PDB), filed under 1QJA (93). Images were generated using the PyMol software. 
present in BAD, of which one phosphoserine residue is directly adjacent to a $\mathrm{BH} 3$ domain that mediates interactions of BAD with $\mathrm{Bcl}-2$ and $\mathrm{Bcl}-\mathrm{X}_{\mathrm{L}}$. Association with 14-3-3 is thought to sterically mask this motif, retaining BAD in the cytosol (94-96). A subsequent study by Yaffe et al. reported that the interactions between 14-3-3 proteins and various client proteins are mediated via discrete binding motifs (Figure 5).
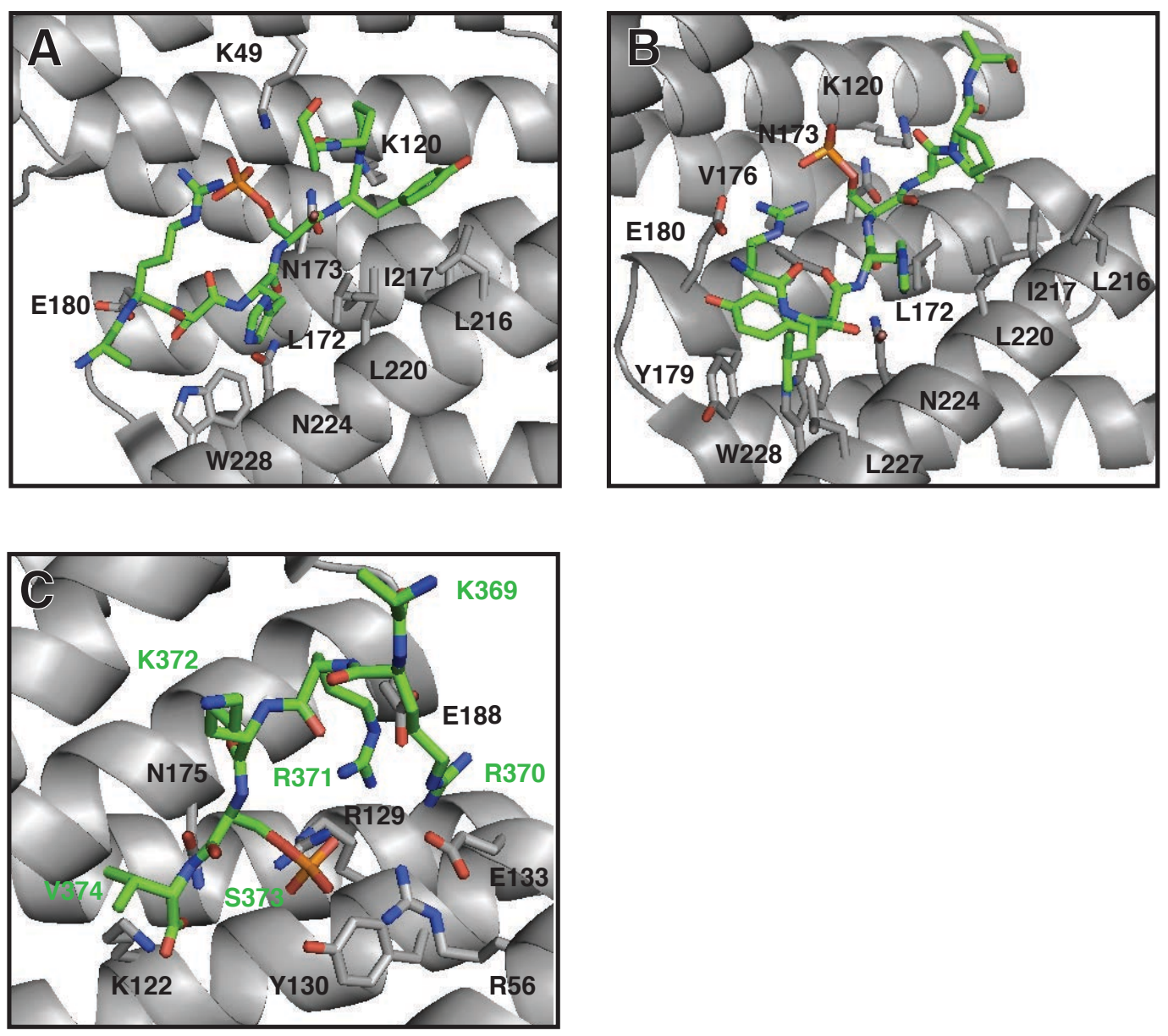

Figure 5: Structural basis of 14-3-3 binding to peptides/proteins containing mode I, mode II and mode III 14-3-3 binding motifs. A: Crystal structure of 14-3-3Z in complex with a peptide containing a mode I 14-3-3 binding motif (ARSHpSYPA). The bound peptide is illustrated in green. The residues involved in peptide binding are indicated as K49, K120, L172, N173, E180, L216, I217, L220, N224 and W228 (93). B: Crystal structure of 14-3-3 3 in complex with a client containing a mode II 14-3-3 binding motif (RLYHpSLPA). The bound peptide is illustrated as green sticks and the phosphoserine residue is depicted as a red and orange tetrahedron, which faces into 


\section{Introduction}

the binding pocket. The residues involved in peptide binding are indicated as $\mathrm{K} 120$, L172, N173, V176, Y179, E180, L216, I217, L220, N224, L227 and W228. In comparison to the residues involve in binding mode I 14-3-3 binding motifs the bound mode II peptide is mainly stabilized by hydrophobic interaction with the inside of the 14-3-3 binding groove (93). C: Crystal structure of $14-3-3 \sigma$ in complex with a TASK3-derived hexapetide. This hexapeptide contains a mode III 14-3-3 binding motif (KRRKSV). The bound peptide is depicted as green sticks and the C-terminal residues of TASK-3 are labeled accordingly, K369, R370, R371, K372, S373 and V374. Residues involve in the binding of this mode III peptide are labeled as R56, K122, R129, Y130, E133, N175 and E188. A comparison between mode I, mode II and Mode III demonstrates that binding of mode I and mode II is chiefly facilitated by interactions formed between the hydrophobic part of the peptide backbone and hydrophobic side chains (93). In contrast, the binding of mode III peptides is mainly stabilized by intricate interactions formed between the phosphoserine residue and multiple residues on the inside of the 14-3-3 binding groove (107). These observations demonstrate that the previous phosphorylation of a mode III binding motif is essential and it further demonstrates that 14-3-3 can bind mode I and mode II binding motifs in a phosphorylation independent manner. The crystal structures were obtained from the Protein Data Bank in Europe, filed under PDB: 1QJA, 1QJB and $3 P 1 N(93,107)$.

The two most commonly found motifs were designated mode I, $\mathrm{RSX}(\mathrm{pS} / \mathrm{pT}) \mathrm{XP}$ and mode II, RXФX(pS/pT)XP, with $\mathrm{X}$ corresponding to any amino acid, pS and pT corresponding to a phosphorylated serine or threonine residue and $\Phi$ corresponding to any hydrophobic amino acid (13). In 2002 several groups reported the association of 14-3-3 proteins with phosphorylated C-terminal peptides of two pore-domain potassium channels (K2P) $(17,18)$. These observations emphasized the role of 14-3-3 in intracellular protein transport of $\mathrm{K}^{+}$-channels. The $\mathrm{C}$-terminal binding motif was subsequently characterized as a mode III 14-3-3 binding motif $\operatorname{RXSX}_{(1-2)^{-}}$ $\mathrm{COOH}$ ), which operates exclusively in a phosphorylation dependent manner $(14,15)$. Although 14-3-3 binding is most often dependent on the phosphorylation state of its client protein, some exceptions are known, where 
bind 14-3-3 binds in a phosphorylation independent manner. A study by Michelsen et al. (2006) demonstrated that the C-terminus of the inward rectifying, ATP-dependent $\mathrm{K}^{+}$-channel Kir6.2 binds to 14-3-3 when presented as a multimer (16), whereas phosphorylation of an adjacent Serine residue abolishes 14-3-3 binding (6). Yang et al. (1999) demonstrated that R18 binds 14-3-3 with high affinity in a phosphorylation independent manner, emphasizing the use of R18 as an inhibitor that interferes with the binding of all 14-3-3 isoforms (98).

In general, 14-3-3 proteins engage in various crucial cellular processes and the selected examples mentioned above represent only a small fraction of all the processes 14-3-3 proteins are involved in. The most important of which are: cell cycle control, apoptosis, membrane protein trafficking, signaltransduction and cell growth (99). These examples should provide a broad overview on the action of 14-3-3 proteins in a cellular context.

\section{Structure of 14-3-3 proteins}

14-3-3 proteins compose a family of highly conserved homo- and heterodimeric proteins, which are abundantly expressed in eukaryotic cells $(79,80)$. In vertebrates seven genes give rise to 10 distinct 14-3-3 isoforms (a, $\beta, \gamma, \delta, \varepsilon, \zeta, \eta, \theta, \tau, \sigma$ ), where three isoforms, 14-3-3a, 14-3-3ठ and 14-3$3 \theta$, represent phosphorylated variants of $14-3-3 \beta, 14-3-3 \zeta$ and $14-3-3 \tau$ respectively (13). Several reports demonstrated that all 14-3-3 isoforms could form homo- and heterodimers, except 14-3-30, in vitro and in vivo (72, 100 102). Furthermore, Gu et al. (2006) and Woodcock et al. (2003) demonstrated that the phosphorylation of serine residues at the dimerization interface influences the dimerization process, allowing for a regulated formation of homo- and heterodimeric 14-3-3 proteins $(101,102)$. Furthermore these reports emphasize a functional role of 14-3-3 monomers in vivo. A subsequent study by Yang et al. (2006) confirmed the existence of monomeric 14-3-3 proteins as part of a highly dynamic equilibrium between monomeric, dimeric and heterodimeric 14-3-3 proteins (Figure 6) (97). 
A

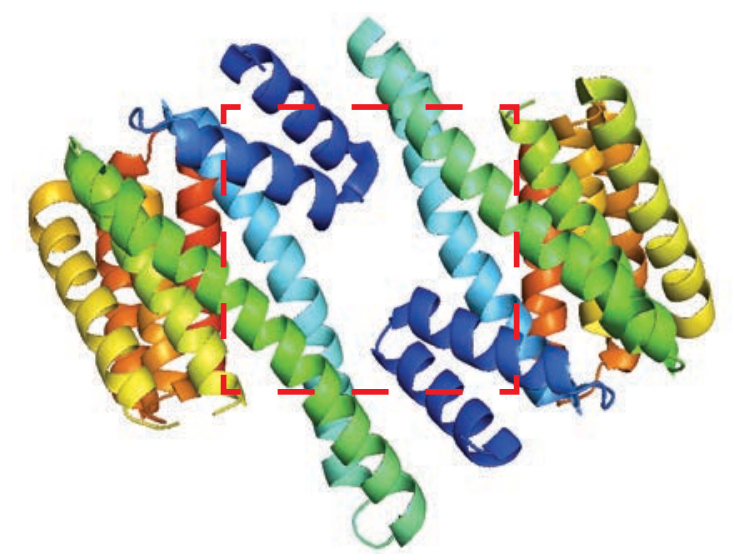

B

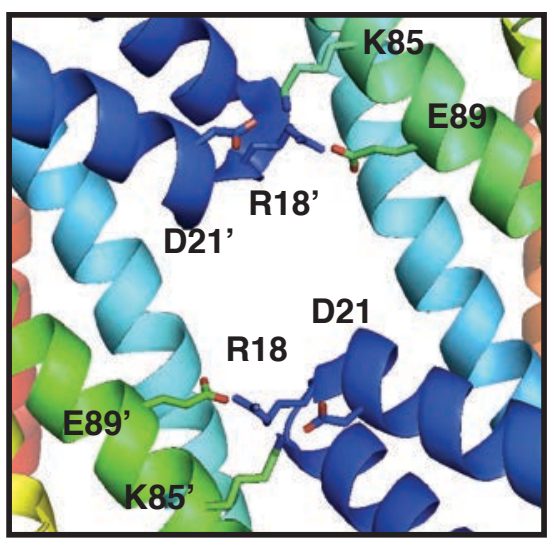

C
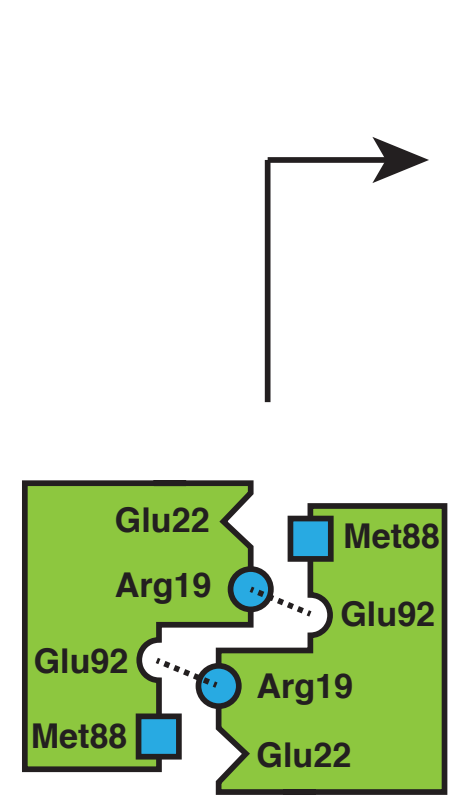

14-3-3 $\varepsilon$ homodimer

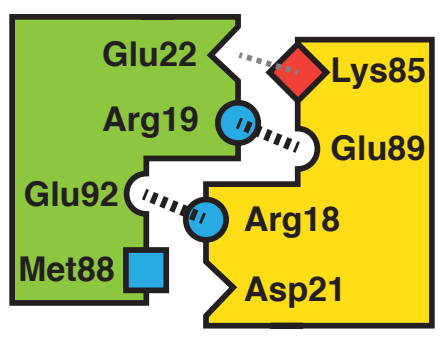

14-3-3 $\varepsilon / \zeta$ heterodimer

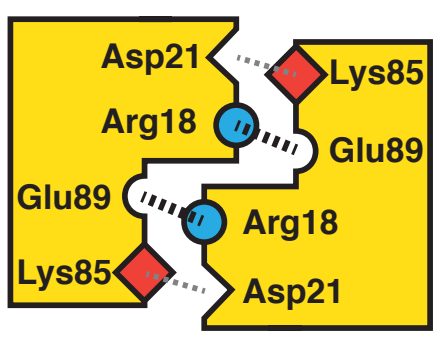

14-3-3द homodimer

Figure 6: $14-3-3$ proteins form homo- and heterodimers in vivo and in vitro. A: Overview of the crystal structure of the 14-3-3 3 homodimer (93). The dimerization interface is framed with a dashed red line. B: Enhanced view of the dimerization interface of 14-3-3द. Salt-bridges formed between residues in the a-helices $a A, a B$ and $\mathrm{aD}$ allow for the formation of a stable homodimer (93). C: Mechanism of 14-3-3 heterodimer formation: Two residues are conserved among all 14-3-3 isoforms. Interactions between these residues are sufficient to form a functional 14-3-3 dimer. 
These residues are Arg19 and Glu92 in 14-3-3ع and the corresponding amino acids

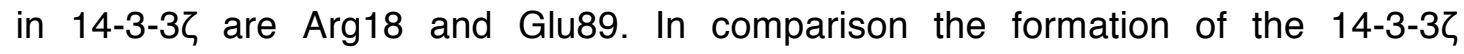
heterodimer is supported by salt-bridges formed between two additional residues, Asp21 and Lys85. 14-3-3e possesses a glutamine and methionine residues at positions equivalent to Asp21 and Lys85 in 14-3-3ろ, which do not form salt-bridges and are not involved in the dimer formation (97). A heterodimer formed between 14$3-3 \varepsilon$ and $14-3-3 \zeta$ was found to form an additional salt-bridge between Glu22 and Lys85. Dashed black lines: salt-bridges found and confirmed between these residues. Dashed grey line: additional salt-bridges that can contribute to the formation of a 14-3-3 homo-, or heterodimer, mechanism adapted by Yang et al. (2006) (97). The crystal structures were obtained from the Protein Data Bank in Europe, filed under PDB: 1QJA (93).

The first crystal structures of $14-3-3 \zeta, 14-3-3 \sigma$ and $14-3-3 \tau$ revealed the remarkable structural conservation between different 14-3-3 isoforms (13, 103, 104). Each 14-3-3 monomer is comprised of nine a-helices (aA - al), of which three helices ( $a A, a C$ and $a D)$ are involved in the formation of the 14-33-dimer interface The horseshoe-shaped amphipathic binding-groove is formed by helices $\mathrm{aE}, \mathrm{aG}$ and al (105). In a ligand bound state residues present in these helices form intricate interactions with the accommodated phosphopeptide, or protein. The sequence alignment of all mammalian 14-3-3 isoforms illustrates the high degree of sequence conservation of the amphipathic binding-groove (the binding groove is formed by helices aE, aG and al) (13). Crystal structures reported for all mammalian 14-3-3 proteins in their ligand bound state confirmed that interactions are facilitated by equivalent residues present in all mammalian 14-3-3 isoforms (13, 93, 105, 106). A study by Yang et al. (2006) further elucidated the structural basis of protein-protein interactions of all known mammalian 14-3-3 isoforms, demonstrating that the 14-3-3 monomer/dimer undergoes a conformational change from an open (ligand-unbound) state to a closed (ligand-bound) state upon binding of a ligand peptide, or protein. This mechanism is thought to allow for rapid adaption of the 14-3-3 homo-, or heterodimer to size, structure 


\section{Introduction}

and phosphorylation state of the client peptide/protein (97). Figure 7 illustrates the structural difference between ligand-unbound and ligand unbound state.

A

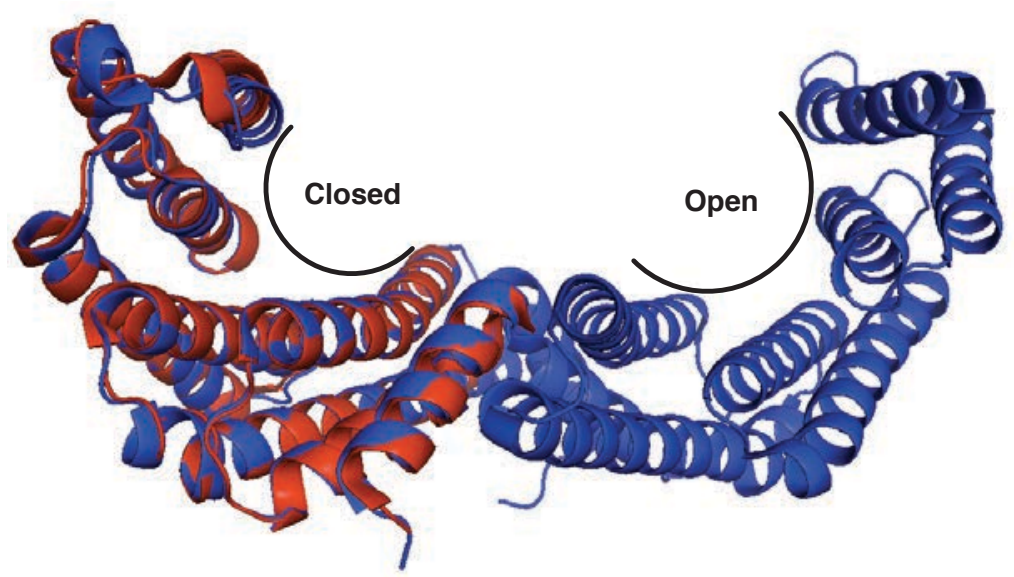

B

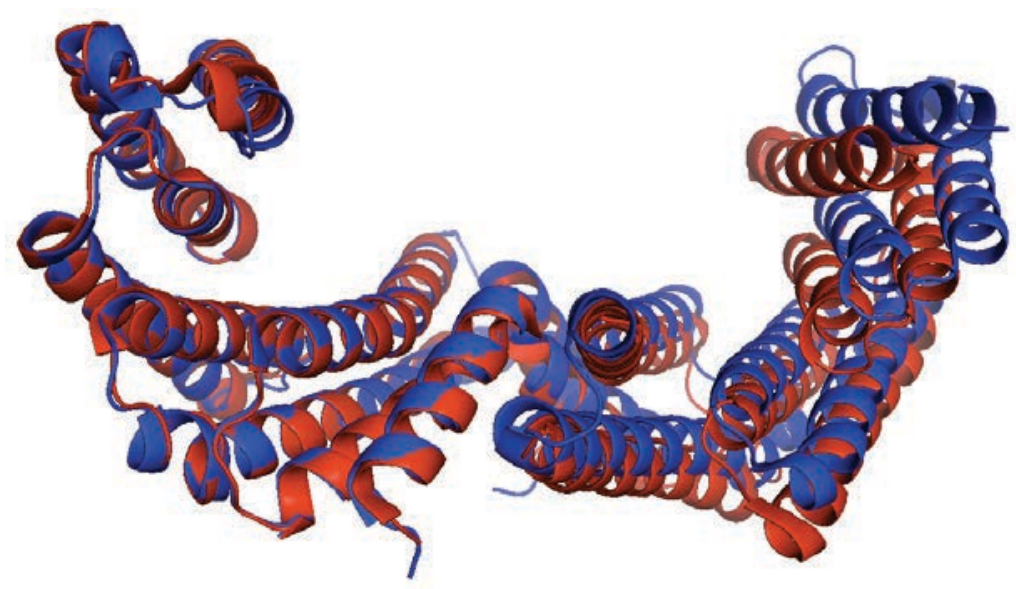

Figure 7: Superimposition of two crystal structures of $14-3-3 \beta$ in a ligand bound state (red) and a ligand unbound state (blue). A: An overlay of two crystal structures demonstrating the differences in the conformation 14-3-3 adopts before and after binding of a peptide substrate. Shown in red is a 14-3-3 $\beta$ monomer bound to a peptide derived from the Pseudomonas aeruginosa ExoS (peptide hidden) (97). Shown in blue is a 14-3-3 $\beta$ homodimer in its apo-form (PDB entry: 4DNK). B: Superimposition of two crystal structures of a $14-3-3 \beta$ dimer in a ligand bound and 
unbound state. The major structure displacement between ligand bound (red) and unbound state (blue) can be observed. The crystal structures were obtained from the Protein Data Bank in Europe, filed under PDB: 2C23 (97) and PDB entry: 4DNK.

A recent report by Anders et al. (2013) gained insight into the structural basics of 14-3-3 binding to peptides containing mode III 14-3-3 consensus sites. A crystal structure of $14-3-3 \sigma$ in complex with a TASK-3-derived hexapeptide demonstrated that peptide binding depends on intricate interactions formed between the inner side of the 14-3-3 binding groove and the accommodated phosphate group, indicating that a deletion of the corresponding residue will also lead to a loss of 14-3-3 binding (107). Although the structural basis of 143-3 binding to mode I, mode II and mode III consensus sites is well understood the following question remains: What determines the binding properties of a 14-3-3 isoform to a given 14-3-3 client and how can the need for seven distinct 14-3-3 isoforms be explained? 
Introduction 


\section{Aims of this thesis}

The antagonistic action of COPI and 14-3-3 regulates the cell surface expression of TASK-1 and TASK-3. Whereas the protein-protein interactions involved in TASK-trafficking have not been described quantitatively yet.

1) The first aim of this study is to investigate interactions between all seven mammalian 14-3-3 isoforms and TASK-derived C-terminal peptides quantitatively. A comparison of binding parameters obtained for TASK-1derived peptides and TASK-3-derived peptides will yield further information on the impact of small sequence differences between TASK-1 and TASK-3 on 14-3-3 binding.

Current literature reports that 14-3-3-binding to TASK-1 and TASK-3 is lost upon truncation of the C-terminus $(11,17,18)$, or mutation of the conserved serine residue, which is part of a mode III 14-3-3 binding motif. Both modifications

2) The second aim of this study is to compare the effect of truncation and mutation of the mode III 14-3-3 binding motif quantitatively. A detailed analysis of the obtained binding parameters will either confirm previous reports, or allow for a more detailed assessment of the underlying mechanism that leads to the reported observations.

Based on a report by Zuzarte et al. (2009), the underlying mechanism of TASK-1 and TASK-3 cell surface expression can be examined employing reporter protein assays (11).

3) The third aim of this thesis is to study the functional consequence of mutation and truncation of the C-termini of TASK-1 and TASK-3 in vivo. Therefore, I wished to study the cell surface expression of different reporter 


\section{Aims}

proteins (wild type and mutant reporter proteins) quantitatively and correlate my findings with the previously obtained in vitro binding data. If my observations can be correlated, I will feel confident that the in vitro and in vivo observations are indeed of biological relevance.

Mant et al. (2011) demonstrated that the TASK-1 and TASK-3 C-terminus can be phosphorylated in vitro by PKA (23), but little is known about the phosphorylation state of different channel/protein variants in vivo.

4) The fourth aim of this study is to investigate the phosphorylation state of different reporter protein constructs in vivo. Furthermore, I wish to study the efficiency, by which different recombinantly expressed TASK-derived fusion proteins are phosphorylated in vitro.

Many reports describe the antagonistic role of COPI and 14-3-3 in TASK-1 and TASK-3 cell surface expression based on experiments performed with cellular extracts $(11,18)$. These extracts contain both proteins/protein complexes and signal transduction events, such as phosphorylation, cannot be assessed individually.

5) The final aim of this thesis is to investigate the effect of phosphorylation of TASK-1 and TASK-3 on binding of the COPI vesicle coat. These experiments will yield insight into the mode of action 14-3-3 performs in cellular context and will allow for a direct comparison between TASK-1 and TASK-3 C-termini. 


\section{Material and Methods}

\section{Molecular biology}

A list of all plasmids used for this study can be found in Table 1.

\section{Restriction enzyme digest}

Plasmid DNA and DNA fragments obtained by polymerase chain reaction were digested with one or multiple restriction enzymes.

For plasmid DNA: $1-2 \mu \mathrm{g}$ of the target vector were incubated with 1-5 units of the restriction enzyme in its respective buffer. Depending on the restriction enzyme, the reaction mix was incubated for $60-90$ minutes at $37^{\circ} \mathrm{C}$. To avoid re-annealing of the linearized vector, during ligation, 1 unit of calf intestinal alkaline phosphatase (CIAP) was added to the reaction mix and incubated at $37^{\circ} \mathrm{C}$ for $\sim 10$ minutes. Alkaline phosphatase catalyses the removal of 5' phosphate groups from DNA strands.

For DNA fragments obtained by PCR: The DNA fragments were purified using the Highpure PCR product purification kit (Roche). Each purification step was carried out according to the manufacturers instructions. The restriction enzyme and its respective buffer were added and the reaction mix was incubated at $37^{\circ} \mathrm{C}$ for 60 minutes.

Digested DNA was then supplemented with DNA loading buffer, which contained a dye to visualize the running front during electrophoresis, and loaded into the wells of an agarose gel.

\section{Polymerase chain reaction}

Polymerase chain reaction (PCR) was employed to exponentially amplify DNA fragments encoding proteins of interest, in vitro. For this method a small quantity of template DNA (20 - $100 \mathrm{ng})$ was mixed with a mixture of dNTPs (the $10 \mathrm{mM}$ stock contained $2.5 \mathrm{mM}$ dATP, dTTP, dGTP, dCTP) two 


\section{Material and Methods}

oligonucleotide primers that can hybridize with target regions of the template DNA and flank the region of interest, and a thermostable proof-reading DNA polymerase. The reaction mixture was then subjected to multiple cycles of DNA-melting, annealing of the oligonucleotide primers and extension of the oligonucleotide primers by DNA polymerase until sufficient DNA was made available for further analysis and sub-cloning.

In general a $50 \mu \mathrm{l}$ reaction mix contained $20-100 \mathrm{ng}$ of template DNA, $800 \mu \mathrm{M}$ dNTPs, $3-5$ units of polymerase, $400 \mathrm{nM}$ of each oligonucleotide primer and sterile water to constitute the final volume.

PCR primers were designed with an average length of $26-42$ base pairs. A thermo cycler block with the following program was used to amplify target DNA:

1. 2 min @ $94^{\circ} \mathrm{C}$

2. $30 \mathrm{sec} @ 94^{\circ} \mathrm{C}$

3. $30 \mathrm{sec} @ \mathrm{Tm}-2{ }^{\circ} \mathrm{C}$; where $\operatorname{Tm}\left[{ }^{\circ} \mathrm{C}\right]=4 \mathrm{x}(\mathrm{G}+\mathrm{C})+2 \mathrm{x}(\mathrm{A}+\mathrm{T})$

4. Xmin @ $74{ }^{\circ} \mathrm{C}$; where $\mathrm{X}=$ For every kilo-base of DNA 1 minute amplification

5. Repeat step 2 to 5 for $25-30$ cycles

6. $5 \mathrm{~min}$ at $74^{\circ} \mathrm{C}$

A list of all primers used for PCR can be found in Table 2.

\section{Cloning with phosphorylated oligonucleotides}

The ligation of DNA requires 5'-phosphate groups that can be linked to the unphosphorylated 3'-end of another strand of DNA. Regarding this property, cloning of short synthetic, phosphorylated DNA fragments into dephosphorylated recipient vectors can be employed to reduce the background during cloning.

Phosphorylated oligonucleotides encoding the last 15 amino acids of TASK-1, TASK-3 and their respective mutants were annealed and ligated into the respective vector. In general, $20 \mu$ annealing mix contained $8 \mu \mathrm{l}$ 
oligonucleotide 1 (FP), $8 \mu$ l oligonucleotide 2 (RP, where FP and RP can hybridize) and $4 \mu \mathrm{l} 5 \mathrm{x}$ annealing buffer (200 mM Tris- $\mathrm{HCl}, 100 \mathrm{mM} \mathrm{MgCl}$, $250 \mathrm{mM} \mathrm{NaCl}, \mathrm{pH}$ 7.5). The annealing mixture was incubated at $90^{\circ} \mathrm{C}$ for $\sim 5$ minutes. Following melting, the reaction mixture was steadily cooled down to $37^{\circ} \mathrm{C}$ and transferred to ice until further use. For ligation into the recipient vector the annealing mixture was diluted 1:100, 1:1,000 and 1:10,000 with sterile water.

A list of phosphorylated oligonucleotides used in this study can be found in Table 3.

\section{Ligation}

In general $30 \mu$ ligation mix contained 50-100 ng of linearized cloning vector, a 3-10 fold molar excess of linearized insert (PCR product and DNA fragments obtained by restriction digest) or diluted annealed oligonucleotide mixture, $1-2$ units of T4DNA ligase, $3 \mu \mathrm{l}$ of $10 \mathrm{x}$ ligation buffer and sterile water to constitute the final volume. The reaction mix was incubated at $18^{\circ} \mathrm{C}$ for 16 hours. The ligase was subsequently heat inactivated at $65^{\circ} \mathrm{C}$.

\section{Agarose gel electrophoresis}

Agarose gel electrophoresis was utilized to separate DNA fragments based on their size for later purification, to halt the progression of restriction enzyme or phosphatase treatment and to determine the yield of a PCR and or restriction enzyme digest. The DNA samples were mixed with loading buffer, containing a dye to mark the running front during gel electrophoresis, and loaded into the wells of the agarose gel. Gels were electrophoresed at $80-100 \mathrm{~V}$ (constant Voltage, small gels) or $160-200 \mathrm{~V}$ (constant Voltage, large gels). A DNA ladder was co-electrophoresed to determine the size of the DNA fragments. All gels were cast with SafeView, a fluorescent dye with an emission wavelength of $\sim 490 \mathrm{~nm}$. Gels containing $1-2 \%$ percent agarose were used 


\section{Material and Methods}

to separate DNA fragments respectively to their size (1\% gels for bigger fragments, $2 \%$ gels for smaller fragments).

\section{Purification of DNA fragments from agarose gels}

Following agarose gel electrophoresis, bands containing DNA fragments of estimated size were excised and weighed. The excised bands were purified using the Highpure PCR product purification kit (Roche). Therefore, each band was resuspended in binding buffer ( $3 \mathrm{M}$ guanidine-thiocyanate, $10 \mathrm{mM}$ Tric- $\mathrm{HCl}, 5 \%$ ethanol $(v / v), \mathrm{pH} 6.6$ ) (3 times the weight of the excised agarose piece) and melted at $55^{\circ} \mathrm{C}$ for $\sim 10$ minutes. The mixture was supplemented with isopropanol and transferred to a purification column, containing a silica matrix. The column was washed twice to purge residual contaminants from the system with washing buffer $(20 \mathrm{mM} \mathrm{NaCl}, 2 \mathrm{mM}$ Tris- $\mathrm{HCl}, 80 \%$ ethanol $(v / v), \mathrm{pH} 7.5)$ and dried by centrifugation. Bound DNA was eluted with elution buffer (10 mM Tris-HCl, $\mathrm{pH} 8.5$ ).

\section{Transformation of bacterial cells by electroporation}

$1.5 \mu \mathrm{l}$ of heat inactivated ligation mix or $\sim 0.5 \mathrm{ng}$ of plasmid DNA sample were mixed with $50 \mu \mathrm{l}$ of electro-competent DH5alpha cells (for amplification of plasmid DNA), or electro-competent BL21(pREP4) cells (for protein expression) and transferred to a pre-cooled electroporation cuvette. The electroporation unit (Gene Pulser, BioRad) was pre-set at $25 \mu \mathrm{F}$ and $2.5 \mathrm{kV}$. The pulse controller was set to $400 \Omega$. One pulse was applied to the cell suspension at the above settings. The cuvette was removed and $1 \mathrm{ml}$ of SOC medium (20 g/l tryptone, $5 \mathrm{~g} / \mathrm{l}$ yeast extract, $0.5 \mathrm{~g} / \mathrm{l} \mathrm{NaCl}, 2.5 \mathrm{mM} \mathrm{KCl,} 10 \mathrm{mM}$ $\mathrm{MgCl}_{2}, 20 \mathrm{mM}$ glucose) pre-warmed to $37^{\circ} \mathrm{C}$ was added. The cells were resuspended with a Pasteur pipette and transferred to an Eppendorf cup, followed by incubation at $37^{\circ} \mathrm{C}$ for 30 minutes. For ligations, the entire suspension was plated onto LB plates containing selective antibiotics. For retransformations (plasmid amplification, or later protein expression) $100 \mu \mathrm{l}$ of the suspension was plated onto LB plates containing selective antibiotics. 


\section{Amplification of plasmid DNA}

Single colonies were picked and $5 \mathrm{ml}$ (small scale) or $100 \mathrm{ml}$ (large scale) LBmedium supplemented with the appropriate antibiotic were inoculated. Cultures were incubated at $37^{\circ} \mathrm{C}$ for $14-16$ hours. Cells were harvested by centrifugation and plasmid DNA was extracted. DNA extraction was carried out according to the manufacturers instructions (NucleoSpin Plasmid, $5 \mathrm{ml}$ cultures; NucleoBond Xtra Midi, 100 ml cultures; Macherey-Nagel).

\section{Protein expression}

MBP-tagged 14-3-3 proteins and GST-fused TASK-1 and TASK-3 C-termini were expressed in the Escherichia coli strain BL21(pREP4). All expressions were performed in 2YT medium (16 g/l Bacto tryptone, $10 \mathrm{~g} / \mathrm{l}$ Bacto yeast extract, $5 \mathrm{~g} / \mathrm{l} \mathrm{NaCl}, 30 \mathrm{mM} \mathrm{K}_{2} \mathrm{HPO}_{4}, 2 \%$ Glycerol, $\mathrm{pH}$ 7.0) supplemented with the appropriate antibiotics and carried out at $37^{\circ} \mathrm{C}$ incubation temperature. $\mathrm{A}$ starter culture grown over night in 2YT medium supplemented with the appropriate antibiotics was diluted to an $\mathrm{OD}_{600}=0.2$ (1L) and protein expression was induced upon addition of IPTG (final concentration $1 \mathrm{mM}$ ) at $\mathrm{OD}_{600}=0.8$. The cells were induced for $3 \mathrm{~h}$ at $37^{\circ} \mathrm{C}$ and harvested by centrifugation at $\sim 4,000 \times \mathrm{g}$ for 20 minutes, washed with water and stored at $20{ }^{\circ} \mathrm{C}$ until further use.

\section{Protein purification}

Cells expressing MBP-tagged 14-3-3 proteins were harvested, washed with water, resuspended in MBP-column buffer $(150 \mathrm{mM} \mathrm{NaCl}, 5 \mathrm{mM} \mathrm{MgCl}$, $50 \mathrm{mM}$ Tris- $\mathrm{HCl}, 1 \mathrm{mM}$ PMSF, $\mathrm{pH}$ 7.5) and subjected to ultrasonication. The cell suspensions were sonicated at 40\% amplitude, 6 times for 10 seconds with a recovery period of 30 seconds between each step. The crude cellular lysates were centrifuged at 100,000g (36,000 RPM, Rotor: 55.2Ti, Beckman Coulter) for 30 minutes at $4{ }^{\circ} \mathrm{C}$. In parallel, the affinity matrices (Amylose resin, New England Bio Labs) were equilibrated in MBP-column buffer (4 washing steps) to purge remaining traces of ethanol. After centrifugation, pre- 


\section{Material and Methods}

cleared cell lysates were incubated with the washed affinity matrices for 90 minutes at $4{ }^{\circ} \mathrm{C}$ while rotating. The affinity matrices were then loaded onto purification columns and washed 6 times with MBP-column buffer to remove contaminants. Following column wash, matrix-bound proteins were eluted with $10 \mathrm{ml}$ MBP-column buffer supplemented with $20 \mathrm{mM}$ D-Maltose. $1 \mathrm{ml}$ fractions were collected and $5 \mu \mathrm{l}$ from each fraction were loaded onto SDS-Page gels (12\%). The gels were electrophoresed at $15 \mathrm{~mA}$ (constant amperage) for 80 minutes, followed by Coomassie staining to identify fractions containing the purified protein. Peak fractions were pooled and concentrated using a Spin-X UF concentrator (Corning). The MBP-tag was proteolytically removed by Factor Xa using the Factor Xa cleavage capture kit (Novagen). Subsequent gel filtration allowed the separation of cleaved MBP and dimeric 14-3-3 proteins. 14-3-3 proteins were gel filtrated at $1 \mathrm{ml} / \mathrm{min}$ flow rate into either SPR (20 mM HEPES, $150 \mathrm{mM} \mathrm{NaCl,} \mathrm{0.005 \%} \mathrm{TWEEN-20,} \mathrm{pH} \mathrm{7.5),} \mathrm{or} \mathrm{FP}$ buffer (20 mM MOPS, $150 \mathrm{mM} \mathrm{NaCl}, 0.005 \%$ CHAPS, pH 7.5) using a Superdex 75pg Hiload 26/60 column. Following gel filtration, proteins were concentrated and stored on ice until further use.

GST-tagged proteins were expressed in a similar manner to MBP-tagged proteins and purification steps were carried out as described above in GSTbreaking buffer containing $150 \mathrm{mM}$ KOAc, $5 \mathrm{mM} \mathrm{Mg}(\mathrm{OAc})_{2}, 20 \mathrm{mM}$ HEPES, $1 \mathrm{mM}$ EDTA, $1 \mathrm{mM}$ DTT, $1 \mathrm{mM}$ PMSF (during lysis) $\mathrm{pH} 6.5$ using glutathione sepharose as an affinity matrix (glutathione sepharose 4FastFlow, GE Healthcare). Proteins were eluted with GST-breaking buffer supplemented with $20 \mathrm{mM}$ glutathione, $\mathrm{pH}$ 8.5. Purified GST-fused proteins were gel filtrated into 1x PBS, pH 7.0 and subsequently concentrated, snap frozen and stored at $-80^{\circ} \mathrm{C}$.

\section{SDS-PAGE}

High percentage gels were cast to separate small molecular weight proteins and low percentage gels were cast to better separate proteins of higher molecular weight. In general resolving gels of $12 \%$ were cast. The stacking 
gel (4\%) was prepared with a Tris ${ }^{*} \mathrm{HCl}$ buffer at $\mathrm{pH} 6.8$, whereas the resolving gel was cast with $\mathrm{Tris}^{*} \mathrm{HCl} \mathrm{pH} 8.8$ to allow concentration of SDS denatured proteins at the interface and to also allow the formation of a narrow starting zone of migration. The height of the stacking gel was always maintained at a quarter of the height of the resolving gel. Gels were electrophoresed at $15 \mathrm{~mA}$ (constant amperage) for 80 minutes.

\section{Phos-tag SDS-PAGE}

To retard the migration of phosphorylated proteins phos-tag SDS-PAGE was employed. Standard SDS-PAGE gels (10\%) were supplemented with $50 \mu \mathrm{M}$ phos-tag acrylamide and $50 \mu \mathrm{M} \mathrm{MnCl}_{2}$. A dinuclear metal complex is formed between manganese-ions $\left(\mathrm{Mn}^{2+}\right)$ and the acrylamide linked 1,3-bis[bis(pyridin2-ylmethyl)amino]propan-2-olato moiety (phos-tag acrylamide, in gel). This complex is bound by phosphate groups of phosphorylated proteins during electrophoresis retarding their migration, which results in a distinct band shift between phosphorylated and unphosphorylated proteins. For further analysis gels were stained with coomassie staining buffer solution.

\section{Electro blotting and Western blot detection}

Following gel electrophoresis, gels were transferred onto nitrocellulose membranes (Amersham Hybond-ECL, GE Healtcare) using a TE22 Mighty Small Transfer Tank. The gels were placed on a pre-wetted membrane and sandwiched between two sheets of blotting paper and two sponge pads. All the individual components were pre-equilibrated in transfer buffer. This set-up was held in place by a blotting cassette and placed in the blotting tank (with the membrane directed towards the anode). The tank was filled with transfer buffer (25 mM Tris, 192 mM Glycine, pH 8.3). Phostag SDS-PAGE gels were washed twice with transfer buffer, which was supplemented with $100 \mathrm{mM}$ EDTA, with intervals of 15 minutes between each wash, prior to transfer. Standard SDS-PAGE gels were electroblotted at $60 \mathrm{~V}$ with the current limited to $0.5 \mathrm{~A}$ for 80 minutes. Phostag SDS-PAGE gels were electroblotted at $15 \mathrm{~V}$ 


\section{Material and Methods}

for 18 hours with the current limited to $75 \mathrm{~mA}$ at $4^{\circ} \mathrm{C}$. Following transfer, the blots of standard SDS-PAGE and Phostag SDS-PAGE gels were stained with Ponceau to visualize the protein bands. The blots were incubated in blocking buffer $(5 \%$ milk powder $(w / v), 25 \mathrm{mM}$ Tris- $\mathrm{HCl} \mathrm{pH} 7.4,135 \mathrm{mM} \mathrm{NaCl}, 3 \mathrm{mM}$ $\mathrm{KCl}, 0.02 \% \mathrm{NP}-40$ ) for 45 minutes at room temperature, or over night at $4^{\circ} \mathrm{C}$. Primary antibodies were diluted in blocking buffer and incubated with the blots for 90 minutes at room temperature. Subsequently, the blots were washed three times with blocking buffer with intervals of 10 minutes between each wash. The fluorescently labeled secondary antibody was diluted in blocking buffer and incubated with the blots for 45 minutes. The blots were washed again three times with blocking buffer with intervals of 10 minutes between subsequent washes, followed by two subsequent washes with blocking buffer without the milk powder. The fluorescently labeled secondary antibodies were detected using an Odyssey LiCOR imaging system (LiCOR, Bad Homburg, Germany).

\section{Coomassie staining of protein gels}

To visualize the protein bands after SDS-PAGE or phos-tag SDS-PAGE, the gels were soaked in fixation buffer (30\% v/v ethanol, $15 \% v / v$ acetic acid) for $\sim 10$ minutes. The gels were then stained with Coomassie staining buffer ( $30 \%$ ethanol, $10 \%$ acetic acid and $0.2 \% \mathrm{w} / \mathrm{v}$ coomassie brilliant blue), boiled in the microwave for $\sim 30$ seconds and incubated for 10 minutes at room temperature and agitated steadily.

\section{In vitro phosphorylation assays}

Purified GST-fused TASK-1 and TASK-3 C-termini and their respective mutants were in vitro phosphorylated using recombinantly expressed cAMP dependent protein kinase A (PKA). In vitro phosphorylation was performed in binding buffer containing $150 \mathrm{mM} \mathrm{NaCl}, 50 \mathrm{mM}$ Tris- $\mathrm{HCl}, 10 \mathrm{mM} \mathrm{MgCl}$ $\mathrm{pH}$ 7.5, supplemented with $200 \mathrm{nM}$ PKA and $100 \mu \mathrm{M}$ ATP, according to Mant et al., 2011 (23). Proteins were either phosphorylated for 20 minutes at room 
temperature or overnight at $4{ }^{\circ} \mathrm{C}$. For overnight phosphorylation an ATP regeneration system was used (energy mix) as described by Yuan et al., 2006 (108). After phosphorylation GST-fused proteins were bound to glutathione sepharose beads. For each experiment $10 \mu \mathrm{g}$ of protein was incubated with $\sim 20 \mu \mathrm{l}$ of bead slurry and incubated at $4{ }^{\circ} \mathrm{C}$ for 20 minutes, rotating. The bead slurry was subsequently transferred to a column and washed 4 times with binding buffer. The proteins were eluted with $75 \mu \mathrm{l}$ $1 \times$ SDS-sample buffer and $10 \%$ of the total volume were loaded onto a SDSor phos-tag SDS-PAGE gel. Gels were electrophoresed at $15 \mathrm{~mA}$ (const. amperage) for 80 minutes and stained with coomassie staining solution.

\section{Surface plasmon resonance (SPR)}

To determine binding parameters between two interacting proteins quantitatively, surface plasmon resonance (SPR) was employed. For this method one of the interacting proteins was immobilized onto the surface (ligand), covalently or by affinity capture, and the second protein (query) serially diluted and injected over the chip surface (analyte), at a constant flow rate. The system consisted of a Reichert SR7500DC biosensor, a pump (SR8500), a diverter valve (SR8600), an auto-sampler (SR8100) and a semiautomatic valve (SR8300). Chip modifications were performed at $15 \mu \mathrm{l} / \mathrm{min}$, whereas all binding experiments were carried out at $40 \mu \mathrm{l} / \mathrm{min}$. All experiments were performed at $20^{\circ} \mathrm{C}$ (chip surface temperature).

For binding studies $\mathrm{HC1000m}$ sensor chips (Xantec Bioanalytics) were modified with a polyclonal anti-GST antibody (Carl Roth) previously described by Knape et al., 2015 (109). The chip surface was activated with $100 \mathrm{mM} \mathrm{N}$ (3-Dimethylaminopropyl)- $N$ '-ethylcarbodiimide hydrochloride (EDC, Sigma) in activation buffer containing $100 \mathrm{mM} \mathrm{N}$-hydroxysuccinimide (NHS), $50 \mathrm{mM}$ 2(N-morpholino)ethane sulfonic acid, pH 5.0 and the ligand, a polyclonal antiGST antibody (Carl Roth) was resuspended in pre-concentration buffer (50 $\mathrm{mM} \mathrm{NaOAc}, \mathrm{pH} 5.0$ ), to a concentration of $30 \mu \mathrm{g} / \mathrm{ml}$, and injected over the activated surface. Following immobilization, the surface was quenched with 
quenching buffer (1 M Ethanolamine, $\mathrm{pH}$ 8.5). After exchange of the running medium, from water to SPR running buffer (20 mM HEPES, $150 \mathrm{mM} \mathrm{NaCl}$, $0.005 \%$ TWEEN-20, $\mathrm{pH} 7.4$ ), three injections of glycine, $\mathrm{pH} 2.1$ were performed. GST-fused TASK-3 C-termini were captured on a surface density of $300 \mu \mathrm{RIU}$ response on the left (ligand) channel and on the right (reference channel) by injecting $150 \mu$ of "ligand" solution (200 nM GST-fused TASK Ctermin) at a flow rate of $15 \mu \mathrm{l} / \mathrm{min}$. The ligand was phosphorylated following its binding to the chip surface (on-chip phosphorylation) using recombinantly expressed protein kinase $A$ (PKA) in SPR buffer (200 nM PKA, 5 mM MgCl , $20 \mathrm{mM}$ HEPES, $150 \mathrm{mM} \mathrm{NaCl}, \quad 0.005 \%$ TWEEN-20). Following phosphorylation the surface was purged of residual PKA and loosely associated proteins with buffer at a flow rate of $100 \mu \mathrm{l} / \mathrm{min}$. A serial dilution of the analyte was subsequently injected over the surface and the response was followed during injection (association phase) and during buffer wash (dissociation phase). The analyte was allowed to associate for 4:30 minutes, dissociation was followed for 7:00 minutes (injected volume $=250 \mu \mathrm{l}$ ). Additional buffer injections were performed, analogous to the analyte injection, which served as a secondary reference. For injections of smaller volume the time of association and dissociation were scaled down accordingly. The obtained data was further processed with Scrubber 2.0c. Equilibrium binding was assessed and equilibrium-binding isotherms were plotted and analyzed with Graph Pad Prism 6.0.

\section{Fluorescence polarization (FP)}

Equilibrium binding parameters between fluorescently labeled C-terminal peptides of TASK-1, TASK-3, mutant C-termini and 14-3-3 proteins were evaluated employing fluorescence polarization (FP). The fluorescence polarization assay was set up according to Moll et al., 2006 (110) and Muda et al., 2014 (111).

This method is based on the excitation of the sample with vertically polarized light and an intensity measurement of the emitted light, via a polarizer. The 
ratio between vertically and parallel polarized light is formed and analyzed in a concentration dependent manner.

Peptides were purchased from Peps4Life sciences (Heidelberg, Germany). The lyophilized peptides were pre-dissolved in DMSO $(5 \% v / v$; where $v$ is the final volume required for a $100 \mu \mathrm{M}$ stock solution) and diluted in FP-buffer (20 mM MOPS, $150 \mathrm{mM} \mathrm{NaCl,} \mathrm{0.005 \%} \mathrm{CHAPS,} \mathrm{pH} \mathrm{7.5)} \mathrm{to} \mathrm{a} \mathrm{final} \mathrm{peptide}$ concentration of $100 \mu \mathrm{M})$. For each experiment a $20 \mathrm{nM}$ working stock of each peptide used in this study was prepared. Purified proteins were serially diluted in FP-buffer and $30 \mu \mathrm{l}$ of each dilution were pipetted into the wells of a 384well microtiter plate (Packard, Meriden CT, USA, Optiplate 384, black). Subsequently, $30 \mu \mathrm{l}$ of the peptide working stock (20 nM) was added (diluted in a 1:1 ratio). The fluorescence polarization signal was detected for $2 \mathrm{~s}$ at an excitation wavelength of $485 \mathrm{~nm}$ with an emission FP filter wavelength of 535 $\mathrm{nm}$ and a photomultiplier voltage of 1,100 , using a Fusion ${ }^{\mathrm{TM}}$ a-FP microtiter plate reader at room temperature.

\section{Cell culture - Passaging cells}

COS-7 cell cultures were grown in culture medium (Dulbecco's modified eagle medium [DMEM], Gibco) and periodically checked under the light microscope. The cells were passaged when $60-70 \%$ confluence was reached. Culture medium and trypsin were pre-warmed at $37^{\circ} \mathrm{C}$ in a water bath. All material and surfaces were sterilized with $70 \%$ ethanol before use. The culture medium was carefully removed by aspiration and the cell containing petri dish was washed with $10 \mathrm{ml} 1 \times$ PBS to wash away dislodged cells from the growth medium. $2 \mathrm{ml}$ of trypsin were added and the petri dish was incubated at $37^{\circ} \mathrm{C}$ in a $5 \% \mathrm{CO}_{2}$ atmosphere for 3 minutes. Detaching of the cells was aided by gently tapping the petri dish. The cell suspension was subsequently checked under the light microscope to ensure no visible cell clumps were present within the cell suspension. $8 \mathrm{ml}$ of culture medium was added to stop the trypsin treatment and to transfer the cell suspension to another petri dish. In general cells were transferred to either a $15 \mathrm{ml}$ petri dish (to maintain cells in 


\section{Material and Methods}

culture) or to a 6 well plate for subsequent transfection. In $15 \mathrm{ml}$ dishes cells were seeded up to $30 \%$ confluence, whereas in 6 well plates 250,000 cells were seeded per experiment ( $40 \%$ confluence).

\section{Cell culture - Transient transfection}

COS-7 cells were transfected one day after seeding to allow cells to properly adhere to the well floor. Typically $4 \mu \mathrm{g}$ of DNA were used per well for transient transfection. $150 \mu \mathrm{l}$ of Opti-MEM (serum free medium, Gibco) was mixed with $10 \mu \mathrm{l}$ FuGENE and incubated for $5 \mathrm{~min}$ a room temperature. To the mix $4 \mu \mathrm{g}$ of DNA was added and equilibrated at room temperature for 15 minutes. The existing culture medium from each well containing COS-7 cells to be transfected was aspirated and each well was washed once with $2 \mathrm{ml} 1 \times$ PBS. $2 \mathrm{ml}$ of pre-warmed culture medium was added to the DNA-FuGENE transfection mix and transferred to the respective wells of the 6-well plate. The cells were incubate at $37^{\circ} \mathrm{C}$ in a controlled environment containing $5 \% \mathrm{CO}_{2}$. Transfected cells were harvested 36 hours after treatment.

\section{Flow cytometry}

COS-7 cells expressing reporter proteins, which consist of the extracellular domain of CD8, an eCFP fluorophore and the last 15 amino acids of either TASK-1, TASK-3 or various mutant versions of each C-terminus, were harvested $36 \mathrm{~h}$ after transfection. Cells were washed once with $1 \times$ PBS to remove the growth medium, treated with trypsin for 3 minutes at $37^{\circ} \mathrm{C}$ and resuspended in culture medium (Dulbecco's modified eagle medium [DMEM], Gibco). The cell suspensions were centrifuged for $3 \mathrm{~min}$ at $300 \mathrm{xg}$ and resuspended in culture medium. Primary antibody, a monoclonal anti-CD8 antibody (Sigma, St Louis MO, USA, C7423), was diluted in $1 \times$ PBS and added to each well of the 6 well plate. The cells were incubated for $60-90$ minutes with primary antibody on ice. Following incubation, cells were washed and resuspended in culture medium. The secondary antibody (Alexa 647, Invitrogen, Waltham MA, USA) was diluted in 1 x PBS and added to the cell 
suspension, followed by 30 minutes incubation on ice. The cells were washed twice with culture medium, resuspended in $1 \times$ PBS and transferred to FACS tubes for further analysis.

Flow cytometry was performed with a FACS Calibur ${ }^{\mathrm{TM}}$ flow cytometer and FACS DIVA ${ }^{\mathrm{TM}}$ software (BD Biosciences, Heidelberg, Germany). Cells were counted and cell surface expression of the reporter protein, stained by the monoclonal anti-CD8 antibody, was assessed. Differences of relative cell surface expression between reporters were evaluated by forming the ratio between the mean intensity of the anti-CD8 staining (surface population of the reporter protein) and the mean intensity of the CFP signal (total amount of reporter protein expressed).

\section{In vivo phosphorylation assays}

COS7 cells were transiently transfected with $5 \mu \mathrm{g}$ DNA encoding the respective CD8 reporter protein. Cells were harvested 36 hours after transfection, washed twice with 1xPBS and resuspended in membrane preparation buffer $(50 \mathrm{mM} \mathrm{NaCl}, 0.32 \mathrm{M}$ sucrose, $2 \mathrm{mM}$ EDTA, $20 \mathrm{mM}$ HEPES, pH 7.4, cOmplete EDTA-free protease inhibitor cocktail (Roche, Mannheim, Germany) and $50 \mu \mathrm{M}$ PKA inhibitor $\mathrm{H}-89$ dihydrochloride hydrate (Sigma-Aldrich)). The cell suspension was homogenized using a MICCRA-D-1 homogenizer disperser (ART Prozess- \& Labortechnik, Müllheim, Germany), followed by 15 strokes with a Dounce homogenizer. The homogenate was centrifuged for $30 \mathrm{~min}$ at $100,000 \mathrm{xg}$ and the insoluble fraction was separated. The obtained pellet was solubilized in $250 \mu \mathrm{l}$ of solubilization buffer $(1.5 \%$ Triton X-100, 0.75\% Na-deoxycholate, 0.1\% SDS, $50 \mathrm{mM}$ Tris- $\mathrm{HCl}, 100 \mathrm{mM}$ $\mathrm{NaCl}, 5 \mathrm{mM}$ EDTA, $2.5 \mathrm{mM}$ EGTA, pH 7.5) for $30 \mathrm{~min}$ on ice, followed by a second centrifugation step. Subsequently, the samples were precipitated with $12.5 \%$ trichloroacetic acid (TCA), washed with acetone and dried at $37^{\circ} \mathrm{C}$. Dried protein pellets were resuspended in $\lambda$ phosphatase buffer (1× PMP buffer, catalog number P0753, New England Biolabs, Frankfurt, Germany). Two samples were prepared for each reporter protein, of which one sample 


\section{Material and Methods}

was supplemented with 1600 units of $\lambda$-protein phosphatase ( $\lambda$-PPase). Both samples were then incubated for 30 minutes at $30^{\circ} \mathrm{C}$. Following incubation samples were supplemented with 5x SDS loading buffer and loaded into the wells of a SDS-PAGE, or Phostag SDS-PAGE gel.

\section{COPI pull-down experiments}

COPI coat was purified from yeast, as described by Yip and Walz (Yip and Walz, 2011) by Dr. Eric Arakel. $10 \mu \mathrm{g}$ of affinity purified GST-MST27-TASK-1CT15 and GST-MST27-TASK-3-CT15 (10, 112) was phosphorylated by recombinant PKA in phosphorylation buffer containing: $20 \mathrm{mM}$ HEPES pH 6.8, $150 \mathrm{mM}$ KOAc, $5 \mathrm{mM} \mathrm{Mg(OAc)2,} 1 \mathrm{mM}$ EDTA, $1 \mathrm{mM}$ DTT, 2\% glycerol, supplemented with an ATP regeneration system comprising $10 \mathrm{mM}$ phosphocreatine, $0.5 \mathrm{mM}$ ATP, $0.5 \mathrm{mM}$ GTP and $50 \mu \mathrm{g} / \mathrm{ml}$ creatine phosphokinase (108). Following phosphorylation, the bait proteins were immobilized on $\sim 20 \mu \mathrm{l}$ of Glutathione-sepharose. The bead slurry was incubated with phosphorylated GST-fusion proteins for 90 minutes at $4^{\circ} \mathrm{C}$, shaking. COPI was added to the bead slurry as indicated and incubated for a minimum period of $60 \mathrm{~min}$. The bait proteins were subsequently washed four times with phosphorylation buffer. Proteins were eluted with 1x SDS sample buffer containing $100 \mathrm{mM}$ DTT. The samples were analyzed by standard SDS-PAGE and Phostag SDS-PAGE. 
Table 1: Plasmids used in this study.

\begin{tabular}{|c|c|c|}
\hline Plasmid name: & Description/Purpose: & Restriction sites used \\
\hline pGEX-6P-1 & $\begin{array}{l}\text { Recombinant expression of } \mathrm{N}- \\
\text { terminally GST-tagged proteins }\end{array}$ & EcoRI - Notl \\
\hline pGEX-6P-1 TASK1-C15 WT & $\begin{array}{l}\text { Bacterial expression vector } \\
\text { encoding GST-TASK1-C15 WT }\end{array}$ & Insert at EcoRI - Notl \\
\hline $\begin{array}{l}\text { pGEX-6P-1 TASK1-C15 } \\
\text { S392A }\end{array}$ & $\begin{array}{l}\text { Bacterial expression vector } \\
\text { encoding GST-TASK1-C15 } \\
\text { S392A }\end{array}$ & Insert at EcoRI - Notl \\
\hline $\begin{array}{l}\text { pGEX-6P-1 TASK1-C15 } \\
\text { S393A }\end{array}$ & $\begin{array}{l}\text { Bacterial expression vector } \\
\text { encoding GST-TASK1-C15 } \\
\text { S393A }\end{array}$ & Insert at EcoRI - Notl \\
\hline $\begin{array}{l}\text { pGEX-6P-1 TASK1-C15 } \\
\text { S392A_S393A }\end{array}$ & $\begin{array}{l}\text { Bacterial expression vector } \\
\text { encoding GST-TASK1-C15 } \\
\text { S392A_S393A }\end{array}$ & Insert at EcoRI - Notl \\
\hline pGEX-6P-1 TASK3-C15 WT & $\begin{array}{l}\text { Bacterial expression vector } \\
\text { encoding GST-TASK3-C15 WT }\end{array}$ & Insert at EcoRI - Notl \\
\hline pGEX-6P-1 TASK1 dV374 & $\begin{array}{l}\text { Bacterial expression vector } \\
\text { encoding GST-TASK3-C15 } \\
\text { dV374 }\end{array}$ & Insert at EcoRI - Notl \\
\hline pGEX-6P-1 TASK1 S373A & $\begin{array}{l}\text { Bacterial expression vector } \\
\text { encoding GST-TASK3-C15 } \\
\text { S373A }\end{array}$ & Insert at EcoRI - Notl \\
\hline pMAL2CX & $\begin{array}{l}\text { Recombinant expression of } \mathrm{N}- \\
\text { terminally MBP-tagged proteins }\end{array}$ & EcoRI - HindIII \\
\hline pMAL2CX 14-3-3 beta & $\begin{array}{l}\text { Bacterial expression vector } \\
\text { encoding MBP-14-3-3 beta }\end{array}$ & Insert at EcoRI - HindIII \\
\hline pMAL2CX 14-3-3 gamma & $\begin{array}{l}\text { Bacterial expression vector } \\
\text { encoding MBP-14-3-3 gamma }\end{array}$ & Insert at EcoRI - HindIII \\
\hline pMAL2CX 14-3-3 epsilon & $\begin{array}{l}\text { Bacterial expression vector } \\
\text { encoding MBP-14-3-3 epsilon }\end{array}$ & Insert at EcoRI - HindIII \\
\hline pMAL2CX 14-3-3 zeta & $\begin{array}{l}\text { Bacterial expression vector } \\
\text { encoding MBP-14-3-3 zeta }\end{array}$ & Insert at EcoRI - HindIII \\
\hline
\end{tabular}


Material and Methods

\begin{tabular}{|c|c|c|}
\hline pMAL2CX 14-3-3 eta & $\begin{array}{l}\text { Bacterial expression vector } \\
\text { encoding MBP-14-3-3 eta }\end{array}$ & Insert at EcoRI - HindIII \\
\hline pMAL2CX 14-3-3 tau & $\begin{array}{l}\text { Bacterial expression vector } \\
\text { encoding MBP-14-3-3 tau }\end{array}$ & Insert at EcoRI - HindIII \\
\hline pMAL2CX 14-3-3 sigma & $\begin{array}{l}\text { Bacterial expression vector } \\
\text { encoding MBP-14-3-3 sigma }\end{array}$ & Insert at EcoRI - HindIII \\
\hline pcDNA3.1 (zeo+) & $\begin{array}{l}\text { Mammalian expression vector, } \\
\text { used for transfection }\end{array}$ & $\begin{array}{l}\text { BamHI - EcoRI- Notl - } \\
\text { Xbal }\end{array}$ \\
\hline pcDNA3.1 CD8 & $\begin{array}{l}\text { Mammalian expression vector } \\
\text { encoding the extracellular } \\
\text { domain of hCD8 }\end{array}$ & Insert at BamHI - EcoRI \\
\hline pcDNA3.1 CD8-CFP & $\begin{array}{l}\text { Mammalian expression vector } \\
\text { encoding the reporter protein } \\
\text { precursor CD8-CFP }\end{array}$ & Insert at EcoRI- Notl \\
\hline $\begin{array}{l}\text { pcDNA3.1 CD8-CFP- } \\
\text { TASK1-C15 WT }\end{array}$ & $\begin{array}{l}\text { Mammalian expression vector } \\
\text { encoding the reporter protein } \\
\text { CD8-CFP-TASK1-C15 WT }\end{array}$ & Insert at Notl - Xbal \\
\hline $\begin{array}{l}\text { pcDNA3.1 CD8-CFP- } \\
\text { TASK1-C15 S392A }\end{array}$ & $\begin{array}{l}\text { Mammalian expression vector } \\
\text { encoding the reporter protein } \\
\text { CD8-CFP-TASK1-C15 S392A }\end{array}$ & Insert at Notl - Xbal \\
\hline $\begin{array}{l}\text { pcDNA3.1 CD8-CFP- } \\
\text { TASK1-C15 S393A }\end{array}$ & $\begin{array}{l}\text { Mammalian expression vector } \\
\text { encoding the reporter protein } \\
\text { CD8-CFP-TASK1-C15 S393A }\end{array}$ & Insert at Notl - Xbal \\
\hline $\begin{array}{l}\text { pcDNA3.1 CD8-CFP- } \\
\text { TASK1-C15 K389A }\end{array}$ & $\begin{array}{l}\text { Mammalian expression vector } \\
\text { encoding the reporter protein } \\
\text { CD8-CFP-TASK1-C15 K389A }\end{array}$ & Insert at Notl - Xbal \\
\hline $\begin{array}{l}\text { pcDNA3.1 CD8-CFP- } \\
\text { TASK1-C14 dV394 }\end{array}$ & $\begin{array}{l}\text { Mammalian expression vector } \\
\text { encoding the reporter protein } \\
\text { CD8-CFP-TASK1-C14 dV394 }\end{array}$ & Insert at Notl - Xbal \\
\hline $\begin{array}{l}\text { pcDNA3.1 CD8-CFP- } \\
\text { TASK3-C15 WT }\end{array}$ & $\begin{array}{l}\text { Mammalian expression vector } \\
\text { encoding the reporter protein } \\
\text { CD8-CFP-TASK3-C15 WT }\end{array}$ & Insert at Notl - Xbal \\
\hline $\begin{array}{l}\text { pcDNA3.1 CD8-CFP- } \\
\text { TASK3-C15 S373A }\end{array}$ & $\begin{array}{l}\text { Mammalian expression vector } \\
\text { encoding the reporter protein } \\
\text { CD8-CFP-TASK3-C15 S373A }\end{array}$ & Insert at Notl - Xbal \\
\hline
\end{tabular}


Material and Methods

\begin{tabular}{|l|l|l|}
\hline $\begin{array}{l}\text { PCDNA3.1 CD8-CFP- } \\
\text { TASK3-C15 K369A }\end{array}$ & $\begin{array}{l}\text { Mammalian expression vector } \\
\text { encoding the reporter protein } \\
\text { CD8-CFP-TASK3-C15 K369A }\end{array}$ & Insert at Notl - Xbal \\
\hline $\begin{array}{l}\text { pCDNA3.1 CD8-CFP- } \\
\text { TASK3-C14 dV374 }\end{array}$ & $\begin{array}{l}\text { Mammalian expression vector } \\
\text { encoding the reporter protein } \\
\text { CD8-CFP-TASK3-C14 dV374 }\end{array}$ & Insert at Notl - Xbal \\
\hline pCDNA3 CD8 C44TASK3 & $\begin{array}{l}\text { Mammalian expression vector } \\
\text { encoding the CD8-TASK3C44 } \\
\text { reporter protein, used as a } \\
\text { template for PCR of CD8 }\end{array}$ & - \\
\hline pECFP-N1 & $\begin{array}{l}\text { Template for PCR of ECFP to } \\
\text { create CD8-CFP-TASK C- } \\
\text { terminal reporter constructs }\end{array}$ & - \\
\hline
\end{tabular}

Table 2: Primers used for polymerase chain reaction (PCR).

\begin{tabular}{|c|c|c|}
\hline Name & Description & Sequence $\left(5^{\prime}-3^{\prime}\right)$ \\
\hline 14-3-3 beta_FP & $\begin{array}{l}\text { Oligonucleotide used for PCR of } \\
\text { 14-3-3 beta from a pcDNA } \\
\text { template, forward primer }\end{array}$ & $\begin{array}{l}\text { ATATGAATTCATGACAA } \\
\text { TGGATAAAAGTGAG }\end{array}$ \\
\hline 14-3-3 beta_RP & $\begin{array}{l}\text { Oligonucleotide used for PCR of } \\
\text { 14-3-3 beta from a pcDNA } \\
\text { template, reverse primer }\end{array}$ & $\begin{array}{l}\text { ATATAAGCTTTTAGTTCT } \\
\text { СTCССTCCCCAGC }\end{array}$ \\
\hline 14-3-3 gamma_FP & $\begin{array}{l}\text { Oligonucleotide used for PCR of } \\
\text { 14-3-3 gamma from a pcDNA } \\
\text { template, forward primer }\end{array}$ & $\begin{array}{l}\text { ATATGAATTCATGGTGG } \\
\text { ACCCCGAGCAACTGGT } \\
\text { G }\end{array}$ \\
\hline 14-3-3 gamma_RP & $\begin{array}{l}\text { Oligonucleotide used for PCR of } \\
\text { 14-3-3 gamma from a pcDNA } \\
\text { template, reverse primer }\end{array}$ & $\begin{array}{l}\text { ATATAAGCTTTTAGTTGT } \\
\text { TGTTGCCTTCTCCTCC }\end{array}$ \\
\hline 14-3-3 tau FP & $\begin{array}{l}\text { Oligonucleotide used for PCR of } \\
\text { 14-3-3 tau from a pcDNA } \\
\text { template, forward primer }\end{array}$ & $\begin{array}{l}\text { ATATGAATTCATGGAGA } \\
\text { AGACTGAGCTGATC }\end{array}$ \\
\hline
\end{tabular}




\begin{tabular}{|l|l|l|}
\hline 14-3-3 tau RP & $\begin{array}{l}\text { Oligonucleotide used for PCR of } \\
\text { 14-3-3 tau from a pcDNA } \\
\text { template, reverse primer }\end{array}$ & $\begin{array}{l}\text { ATATAAGCTTTTAGTTTT } \\
\text { CAGCCCCTTCTGC }\end{array}$ \\
\hline 14-3-3 sigma FP & $\begin{array}{l}\text { Oligonucleotide used for PCR of } \\
\text { 14-3-3 sigma from a pcDNA } \\
\text { template, forward primer }\end{array}$ & $\begin{array}{l}\text { CCGGAATTCCGGATGG } \\
\text { AGAGAGCCAGTCTGATC } \\
\text { CAG }\end{array}$ \\
\hline 14-3-3 sigma RP & $\begin{array}{l}\text { Oligonucleotide used for PCR of } \\
\text { 14-3-3 sigma from a pcDNA } \\
\text { template, reverse primer }\end{array}$ & $\begin{array}{l}\text { CCCAAGCTTGGGTCAG } \\
\text { CTCTGGGGCTCCTGGG } \\
\text { GAAC }\end{array}$ \\
\hline CD8 FP & $\begin{array}{l}\text { Oligonucleotide used for PCR of } \\
\text { hCD8 from a pcDNA template, } \\
\text { forward primer }\end{array}$ & $\begin{array}{l}\text { CGCGGATCCGCGATGG } \\
\text { CCTTACCAGTGACCGCC } \\
\text { TTG }\end{array}$ \\
\hline CD8 RP & $\begin{array}{l}\text { Oligonucleotide used for PCR of } \\
\text { hCD8 from a pcDNA template, } \\
\text { reverse primer }\end{array}$ & $\begin{array}{l}\text { CCGGAATTCCGGGTTCC } \\
\text { TGTGGTTGCAGTAAAGG }\end{array}$ \\
& $\begin{array}{l}\text { For PCR of CFP from pECFP- } \\
\text { N1, contains a EcoRI restriction } \\
\text { site }\end{array}$ & $\begin{array}{l}\text { CCGGAATTCCGGGTGA } \\
\text { GCAAGGGCGAGGAGCT } \\
\text { GTTC }\end{array}$ \\
\hline CFP FP & $\begin{array}{l}\text { For PCR of CFP from pECFP- } \\
\text { N1, contains a Notl restriction } \\
\text { site }\end{array}$ & $\begin{array}{l}\text { TTTATAGCGGCCGCATA } \\
\text { TTTCTTGTACAGCTCGT } \\
\text { CCATGCC }\end{array}$ \\
\hline
\end{tabular}

Table 3: Phosphorylated oligonucleotides used in this study.

\begin{tabular}{|l|l|l|}
\hline & Purpose & Sequence (5' - 3') \\
\hline GST-TASK1-C15 WT FP & $\begin{array}{l}\text { Phosphorylated oligonucleotide } \\
\text { that encodes the last 15 amino } \\
\text { acids of the TASK1 C-terminus, } \\
\text { used for annealing. }\end{array}$ & $\begin{array}{l}\text { AATTCCACAGTTTATCG } \\
\text { ACATTTCGAGGTCTCAT } \\
\text { GAAGCGAAGAAGCTCA } \\
\text { GTGTAATCGAGC }\end{array}$ \\
\hline $\begin{array}{l}\text { GST-TASK1-C15 WT RP } \\
\text { 5'Notl - EcoRI]3' }\end{array}$ & $\begin{array}{l}\text { Phosphorylated oligonucleotide } \\
\text { that encodes the last 15 amino } \\
\text { acids of the TASK1 C-terminus, } \\
\text { used for annealing. }\end{array}$ & $\begin{array}{l}\text { GGCCGCTCGATTACACT } \\
\text { GAGCTTCTTCGCTTCAT } \\
\text { GAGACCTCGAAATGTCG }\end{array}$ \\
\hline GST-TASK1-C15 S392A FP & $\begin{array}{l}\text { Phosphorylated oligonucleotide } \\
\text { that encodes the last 15 amino } \\
\text { acids of the TASK1 C-terminus, } \\
\text { carrying a mutation at S392A, } \\
\text { used for annealing. }\end{array}$ & $\begin{array}{l}\text { AATTCCACAGTTTATCG } \\
\text { ACATTTCGAGGTCTCAT } \\
\text { GAAGGAAGAGCCTCA }\end{array}$ \\
GTGTAATCGAGC
\end{tabular}


Material and Methods

\begin{tabular}{|c|c|c|}
\hline $\begin{array}{l}\text { GST-TASK1-C15 S392A RP } \\
\text { 5'[Notl - EcoRI]3' }\end{array}$ & $\begin{array}{l}\text { Phosphorylated oligonucleotide } \\
\text { that encodes the last } 15 \text { amino } \\
\text { acids of the TASK1 C-terminus, } \\
\text { carrying a mutation at S392A, } \\
\text { used for annealing. }\end{array}$ & $\begin{array}{l}\text { GGCCGCTCGATTACACT } \\
\text { GAGGCTCTTCGCTTCAT } \\
\text { GAGACCTCGAAATGTCG } \\
\text { ATAAACTGTG }\end{array}$ \\
\hline $\begin{array}{l}\text { GST-TASK1-C15 S393A FP } \\
\text { 5'[EcoRI - Notl]3' }\end{array}$ & $\begin{array}{l}\text { Phosphorylated oligonucleotide } \\
\text { that encodes the last } 15 \text { amino } \\
\text { acids of the TASK1 C-terminus, } \\
\text { carrying a mutation at S393A, } \\
\text { used for annealing. }\end{array}$ & $\begin{array}{l}\text { AATTCCACAGTTTATCG } \\
\text { ACATTTCGAGGTCTCAT } \\
\text { GAAGCGAAGAAGCGCC } \\
\text { GTGTAATCGAGC }\end{array}$ \\
\hline $\begin{array}{l}\text { GST-TASK1-C15 S393A RP } \\
5^{\prime}[\text { Notl - EcoRI]3' }\end{array}$ & $\begin{array}{l}\text { Phosphorylated oligonucleotide } \\
\text { that encodes the last } 15 \text { amino } \\
\text { acids of the TASK1 C-terminus, } \\
\text { carrying a mutation at S393A, } \\
\text { used for annealing. }\end{array}$ & $\begin{array}{l}\text { GGCCGCTCGATTACAC } \\
\text { GGCGCTTCTTCGCTTCA } \\
\text { TGAGACCTCGAAATGTC } \\
\text { GATAAACTGTG }\end{array}$ \\
\hline $\begin{array}{l}\text { GST-TASK1-C15 S392/393A } \\
\text { FP } \\
5^{\prime}[\text { EcoRI - Notl]3' }\end{array}$ & $\begin{array}{l}\text { Phosphorylated oligonucleotide } \\
\text { that encodes the last } 15 \text { amino } \\
\text { acids of the TASK } 1 \text { C-terminus, } \\
\text { carrying mutations at positions } \\
\text { S392/393A, used for annealing. }\end{array}$ & $\begin{array}{l}\text { AATTCCACAGTTTATCG } \\
\text { ACATTTCGAGGTCTCAT } \\
\text { GAAGCGAAGAGCCGCC } \\
\text { GTGTAATCGAGC }\end{array}$ \\
\hline $\begin{array}{l}\text { GST-TASK1-C15 S392/393A } \\
\text { RP } \\
5^{\prime}[\text { Notl - EcoRl]3' }\end{array}$ & $\begin{array}{l}\text { Phosphorylated oligonucleotide } \\
\text { that encodes the last } 15 \text { amino } \\
\text { acids of the TASK1 C-terminus, } \\
\text { carrying mutations at positions } \\
\text { S392/393A, used for annealing. }\end{array}$ & $\begin{array}{l}\text { GGCCGCTCGATTACAC } \\
\text { GGCGGCTCTTCGCTTCA } \\
\text { TGAGACCTCGAAATGTC } \\
\text { GATAAACTGTG }\end{array}$ \\
\hline $\begin{array}{l}\text { GST-TASK3-C15 WT FP } \\
5^{\prime}[\text { EcoRI - Notl }] 3^{\prime}\end{array}$ & $\begin{array}{l}\text { Phosphorylated oligonucleotide } \\
\text { that encodes the last } 15 \text { amino } \\
\text { acids of the TASK3 C-terminus, } \\
\text { used for annealing. }\end{array}$ & $\begin{array}{l}\text { AATTCAGCTTTACCGAC } \\
\text { CACCAGAGGCTGATGA } \\
\text { AACGCCGGAAGTCCGT } \\
\text { TTAAACCTCGAGC }\end{array}$ \\
\hline $\begin{array}{l}\text { GST-TASK3-C15 WT RP } \\
5^{\prime}\left[\text { NotI - EcoRI } 3^{\prime}\right.\end{array}$ & $\begin{array}{l}\text { Phosphorylated oligonucleotide } \\
\text { that encodes the last } 15 \text { amino } \\
\text { acids of the TASK3 C-terminus, } \\
\text { used for annealing. }\end{array}$ & $\begin{array}{l}\text { GGCCGCTCGAGGTTTA } \\
\text { AACGGACTTCCGGCGTT } \\
\text { TCATCAGCCTCTGGTGG } \\
\text { TCGGTAAAGCTG }\end{array}$ \\
\hline
\end{tabular}


Material and Methods

\begin{tabular}{|c|c|c|}
\hline $\begin{array}{l}\text { GST-TASK3 dV374 FP } \\
5^{\prime}[\text { EcoRI - Notl]3' }\end{array}$ & $\begin{array}{l}\text { Phosphorylated oligonucleotide } \\
\text { that encodes the last } 14 \text { amino } \\
\text { acids of the TASK3 C-terminus, } \\
\text { truncated at dV374, used for } \\
\text { annealing. }\end{array}$ & $\begin{array}{l}\text { AATTCAGCTTTACCGAC } \\
\text { CACCAGAGGCTGATGA } \\
\text { AACGCCGGAAGTCCTAA } \\
\text { ACCTCGAGC }\end{array}$ \\
\hline $\begin{array}{l}\text { GST-TASK3 dV374 RP } \\
\text { 5'[Notl - EcoRI]3' }\end{array}$ & $\begin{array}{l}\text { Phosphorylated oligonucleotide } \\
\text { that encodes the last } 14 \text { amino } \\
\text { acids of the TASK3 C-terminus, } \\
\text { truncated at dV374, used for } \\
\text { annealing. }\end{array}$ & $\begin{array}{l}\text { GGCCGCTCGAGGTTTA } \\
\text { GGACTTCCGGCGTTTCA } \\
\text { TCAGCCTCTGGTGGTCG } \\
\text { GTAAAGCTG }\end{array}$ \\
\hline $\begin{array}{l}\text { CD8-CFP-TASK1-C15 WT } \\
\text { FP } \\
5 \text { 'Notl - Xbal]3' }\end{array}$ & $\begin{array}{l}\text { Phosphorylated oligonucleotide } \\
\text { that encodes the last } 15 \text { amino } \\
\text { acids of the TASK1 C-terminus, } \\
\text { used for annealing. }\end{array}$ & $\begin{array}{l}\text { GGCCGCTCACAGTTTAT } \\
\text { CGACATTTCGAGGTCTC } \\
\text { ATGAAGCGAAGAAGCTC } \\
\text { AGTGTAAT }\end{array}$ \\
\hline $\begin{array}{l}\text { CD8-CFP-TASK1-C15 WT } \\
\text { RP } \\
5^{\prime}[\mathrm{Xbal}-\mathrm{Notl}] 3^{\prime}\end{array}$ & $\begin{array}{l}\text { Phosphorylated oligonucleotide } \\
\text { that encodes the last } 15 \text { amino } \\
\text { acids of the TASK1 C-terminus, } \\
\text { used for annealing. }\end{array}$ & $\begin{array}{l}\text { CTAGATTACACTGAGCT } \\
\text { TCTTCGCTTCATGAGAC } \\
\text { CTCGAAATGTCGATAAA } \\
\text { CTGTGAGC }\end{array}$ \\
\hline $\begin{array}{l}\text { CD8-CFP-TASK1 dV394 FP } \\
5^{\prime}[\text { Notl - Xbal }] 3^{\prime}\end{array}$ & $\begin{array}{l}\text { Phosphorylated oligonucleotide } \\
\text { that encodes the last } 14 \text { amino } \\
\text { acids of the TASK1 C-terminus, } \\
\text { truncated at dV394, used for } \\
\text { annealing. }\end{array}$ & $\begin{array}{l}\text { GGCCGCTCACAGTTTAT } \\
\text { CGACATTTCGAGGTCTC } \\
\text { ATGAAGCGAAGAAGCTC } \\
\text { ATAAT }\end{array}$ \\
\hline $\begin{array}{l}\text { CD8-CFP-TASK1 dV394 RP } \\
5^{\prime}[\text { Xbal - Notl }] 3^{\prime}\end{array}$ & $\begin{array}{l}\text { Phosphorylated oligonucleotide } \\
\text { that encodes the last } 14 \text { amino } \\
\text { acids of the TASK1 C-terminus, } \\
\text { carrying at dV394, used for } \\
\text { annealing. }\end{array}$ & $\begin{array}{l}\text { CTAGATTATGAGCTTCT } \\
\text { TCGCTTCATGAGACCTC } \\
\text { GAAATGTCGATAAACTG } \\
\text { TGAGC }\end{array}$ \\
\hline $\begin{array}{l}\text { CD8-CFP-TASK1 S392A FP } \\
5^{\prime}\left[\text { Notl - Xbal } 3^{\prime}\right.\end{array}$ & $\begin{array}{l}\text { Phosphorylated oligonucleotide } \\
\text { that encodes the last } 15 \text { amino } \\
\text { acids of the TASK1 C-terminus, } \\
\text { carrying a mutation at S392A, } \\
\text { used for annealing. }\end{array}$ & $\begin{array}{l}\text { GGCCGCTCACAGTTTAT } \\
\text { CGACATTTCGAGGTCTC } \\
\text { ATGAAGCGAAGAGCCT } \\
\text { CAGTGTAAT }\end{array}$ \\
\hline
\end{tabular}


Material and Methods

\begin{tabular}{|c|c|c|}
\hline $\begin{array}{l}\text { CD8-CFP-TASK1 S392A RP } \\
5 \text { '[Xbal - Notl]3' }\end{array}$ & $\begin{array}{l}\text { Phosphorylated oligonucleotide } \\
\text { that encodes the last } 15 \text { amino } \\
\text { acids of the TASK1 C-terminus, } \\
\text { carrying a mutation at S392A, } \\
\text { used for annealing. }\end{array}$ & $\begin{array}{l}\text { CTAGATTACACTGAGGC } \\
\text { TCTTCGCTTCATGAGAC } \\
\text { CTCGAAATGTCGATAAA } \\
\text { CTGTGAGC }\end{array}$ \\
\hline $\begin{array}{l}\text { CD8-CFP-TASK1 S393A FP } \\
5 \text { '[Notl - Xball]' }\end{array}$ & $\begin{array}{l}\text { Phosphorylated oligonucleotide } \\
\text { that encodes the last } 15 \text { amino } \\
\text { acids of the TASK } 1 \text { C-terminus, } \\
\text { carrying a mutation at S393A, } \\
\text { used for annealing. }\end{array}$ & $\begin{array}{l}\text { GGCCGCTCACAGTTTAT } \\
\text { CGACATTTCGAGGTCTC } \\
\text { ATGAAGCGAAGAAGCG } \\
\text { CCGTGTAAT }\end{array}$ \\
\hline $\begin{array}{l}\text { CD8-CFP-TASK1 S393A RP } \\
5 \text { '[Xbal - Notl]3' }\end{array}$ & $\begin{array}{l}\text { Phosphorylated oligonucleotide } \\
\text { that encodes the last } 15 \text { amino } \\
\text { acids of the TASK1 C-terminus, } \\
\text { carrying a mutation at S393A, } \\
\text { used for annealing. }\end{array}$ & $\begin{array}{l}\text { CTAGATTACACCGGGCT } \\
\text { TCTTCGCTTCATGAGAC } \\
\text { CTCGAAATGTCGATAAA } \\
\text { CTGTGAGC }\end{array}$ \\
\hline $\begin{array}{l}\text { CD8-CFP-TASK1 K389A FP } \\
\text { 5'[Notl - Xbal]3' }\end{array}$ & $\begin{array}{l}\text { Phosphorylated oligonucleotide } \\
\text { that encodes the last } 15 \text { amino } \\
\text { acids of the TASK1 C-terminus, } \\
\text { carrying a mutation at K389A, } \\
\text { used for annealing. }\end{array}$ & $\begin{array}{l}\text { GGCCGCTCACAGTTTAT } \\
\text { CGACATTTCGAGGTCTC } \\
\text { ATGGCCCGAAGAAGCT } \\
\text { CAGTGTAAT }\end{array}$ \\
\hline $\begin{array}{l}\text { CD8-CFP-TASK1 K389A RP } \\
5 \text { '[Xbal - Notl]3' }\end{array}$ & $\begin{array}{l}\text { Phosphorylated oligonucleotide } \\
\text { that encodes the last } 15 \text { amino } \\
\text { acids of the TASK1 C-terminus, } \\
\text { carrying a mutation at K389A, } \\
\text { used for annealing. }\end{array}$ & $\begin{array}{l}\text { CTAGATTACACTGAGCT } \\
\text { TCTTCGGGCCATGAGAC } \\
\text { CTCGAAATGTCGATAAA } \\
\text { CTGTGAGC }\end{array}$ \\
\hline $\begin{array}{l}\text { CD8-CFP-TASK1 } \\
\text { S392/393A FP } \\
\text { 5'[Notl - Xbal]3' }\end{array}$ & $\begin{array}{l}\text { Phosphorylated oligonucleotide } \\
\text { that encodes the last } 15 \text { amino } \\
\text { acids of the TASK } 1 \text { C-terminus, } \\
\text { carrying mutations at positions } \\
\text { S392/393A, used for annealing. }\end{array}$ & $\begin{array}{l}\text { GGCCGCTCACAGTTTAT } \\
\text { CGACATTTCGAGGTCTC } \\
\text { ATGAAGCGAAGAGCCG } \\
\text { CCGTGTAAT }\end{array}$ \\
\hline $\begin{array}{l}\text { CD8-CFP-TASK1 } \\
\text { S392/393A RP } \\
\text { 5'[Xbal - Notl]3' }\end{array}$ & $\begin{array}{l}\text { Phosphorylated oligonucleotide } \\
\text { that encodes the last } 15 \text { amino } \\
\text { acids of the TASK } 1 \text { C-terminus, } \\
\text { carrying mutations at positions } \\
\text { S392/393A, used for annealing. }\end{array}$ & $\begin{array}{l}\text { CTAGATTACACGGCGG } \\
\text { CTCTTCGCTTCATGAGA } \\
\text { CCTCGAAATGTCGATAA } \\
\text { ACTGTGAGC }\end{array}$ \\
\hline
\end{tabular}




\section{Material and Methods}

\begin{tabular}{|c|c|c|}
\hline $\begin{array}{l}\text { CD8-CFP-TASK3-C15 WT } \\
\text { FP } \\
5 \text { '[Notl - Xbal]3' }\end{array}$ & $\begin{array}{l}\text { Phosphorylated oligonucleotide } \\
\text { that encodes the last } 15 \text { amino } \\
\text { acids of the TASK3 C-terminus, } \\
\text { used for annealing. }\end{array}$ & $\begin{array}{l}\text { GGCCGCCAGCTTTACC } \\
\text { GACCACCAGAGGCTGA } \\
\text { TGAAACGCCGGAAGTC } \\
\text { CGTTTAAACCT }\end{array}$ \\
\hline $\begin{array}{l}\text { CD8-CFP-TASK3-C15 WT } \\
\text { RP } \\
5^{\prime}[\mathrm{Xbal}-\mathrm{Notl}] 3^{\prime}\end{array}$ & $\begin{array}{l}\text { Phosphorylated oligonucleotide } \\
\text { that encodes the last } 15 \text { amino } \\
\text { acids of the TASK3 C-terminus, } \\
\text { used for annealing. }\end{array}$ & $\begin{array}{l}\text { CTAGAGGTTTAAACGGA } \\
\text { CTTCCGGCGTTTCATCA } \\
\text { GCCTCTGGTGGTCGGT } \\
\text { AAAGCTGGC }\end{array}$ \\
\hline $\begin{array}{l}\text { CD8-CFP-TASK3 dV374 FP } \\
5^{\prime}[\text { Notl - Xbal }] 3^{\prime}\end{array}$ & $\begin{array}{l}\text { Phosphorylated oligonucleotide } \\
\text { that encodes the last } 14 \text { amino } \\
\text { acids of the TASK3 C-terminus, } \\
\text { truncated at dV374, used for } \\
\text { annealing. }\end{array}$ & $\begin{array}{l}\text { GGCCGCCAGCTTTACC } \\
\text { GACCACCAGAGGCTGA } \\
\text { TGAAACGCCGGAAGTC } \\
\text { CTAAACCT }\end{array}$ \\
\hline $\begin{array}{l}\text { CD8-CFP-TASK3 dV374 RP } \\
5^{\prime}[\text { Xbal - Notl }] 3^{\prime}\end{array}$ & $\begin{array}{l}\text { Phosphorylated oligonucleotide } \\
\text { that encodes the last } 14 \text { amino } \\
\text { acids of the TASK3 C-terminus, } \\
\text { truncated at dV374, used for } \\
\text { annealing. }\end{array}$ & $\begin{array}{l}\text { CTAGAGGTTTAGGACTT } \\
\text { CCGGCGTTTCATCAGCC } \\
\text { TCTGGTGGTCGGTAAAG } \\
\text { CTGGC }\end{array}$ \\
\hline $\begin{array}{l}\text { CD8-CFP-TASK3 S373A FP } \\
5^{\prime}[\text { Notl - Xbal }] 3^{\prime}\end{array}$ & $\begin{array}{l}\text { Phosphorylated oligonucleotide } \\
\text { that encodes the last } 15 \text { amino } \\
\text { acids of the TASK3 C-terminus } \\
\text { carrying a mutation at S373A, } \\
\text { used for annealing. }\end{array}$ & $\begin{array}{l}\text { GGCCGCCAGCTTTACC } \\
\text { GACCACCAGAGGCTGA } \\
\text { TGAAACGCCGGAAGGC } \\
\text { CGTTTAAACCT }\end{array}$ \\
\hline $\begin{array}{l}\text { CD8-CFP-TASK3 S373A RP } \\
5^{\prime}[\text { Xbal - Notl }] 3^{\prime}\end{array}$ & $\begin{array}{l}\text { Phosphorylated oligonucleotide } \\
\text { that encodes the last } 15 \text { amino } \\
\text { acids of the TASK3 C-terminus } \\
\text { carrying a mutation at S373A, } \\
\text { used for annealing. }\end{array}$ & $\begin{array}{l}\text { CTAGAGGTTTAAACGGC } \\
\text { CTTCCGGCGTTTCATCA } \\
\text { GCCTCTGGTGGTCGGT } \\
\text { AAAGCTGGC }\end{array}$ \\
\hline $\begin{array}{l}\text { CD8-CFP-TASK3 K369A FP } \\
5^{\prime}[\text { Notl - Xbal]3' }\end{array}$ & $\begin{array}{l}\text { Phosphorylated oligonucleotide } \\
\text { that encodes the last } 15 \text { amino } \\
\text { acids of the TASK3 C-terminus } \\
\text { carrying a mutation at K369A, } \\
\text { used for annealing. }\end{array}$ & $\begin{array}{l}\text { GGCCGCCAGCTTTACC } \\
\text { GACCACCAGAGGCTGA } \\
\text { TGGCCCGCCGGAAGTC } \\
\text { CGTTTAAACCT }\end{array}$ \\
\hline
\end{tabular}


Material and Methods

\begin{tabular}{|c|c|c|}
\hline $\begin{array}{l}\text { CD8-CFP-TASK3 K369A RP } \\
5^{\prime}[\text { Xbal - Notl }] 3^{\prime}\end{array}$ & $\begin{array}{l}\text { Phosphorylated oligonucleotide } \\
\text { that encodes the last } 15 \text { amino } \\
\text { acids of the TASK3 C-terminus } \\
\text { carrying a mutation at K369A, } \\
\text { used for annealing. }\end{array}$ & $\begin{array}{l}\text { CTAGAGGTTTAAACGGA } \\
\text { CTTCCGGCGGGCCATC } \\
\text { AGCCTCTGGTGGTCGG } \\
\text { TAAAGCTGGC }\end{array}$ \\
\hline $\begin{array}{l}\text { GST-MST27-TASK1-C15 } \\
\text { WT FP } \\
\text { 5'[Clal - Xhol]3' }\end{array}$ & $\begin{array}{l}\text { Phosphorylated oligonucleotide } \\
\text { that encodes the last } 15 \text { amino } \\
\text { acids of the TASK1 C-terminus, } \\
\text { used for annealing. }\end{array}$ & $\begin{array}{l}\text { CGATGGCGGCGGCGGC } \\
\text { TCGGGCGGCGGCGGCT } \\
\text { CTCACAGTTTATCGACA } \\
\text { TTTCGAGGTCTCATGAA } \\
\text { GCGAAGAAGCTCAGTG } \\
\text { TAAC }\end{array}$ \\
\hline $\begin{array}{l}\text { GST-MST27-TASK1-C15 } \\
\text { WT RP } \\
\text { 5'[Xhol - Clal] } 3 \text { ' }\end{array}$ & $\begin{array}{l}\text { Phosphorylated oligonucleotide } \\
\text { that encodes the last } 15 \text { amino } \\
\text { acids of the TASK1 C-terminus, } \\
\text { used for annealing. }\end{array}$ & $\begin{array}{l}\text { TCGAGTTACACTGAGCT } \\
\text { TCTTCGCTTCATGAGAC } \\
\text { CTCGAAATGTCGATAAA } \\
\text { CTGTGAGAGCCGCCGC } \\
\text { CGCCCGAGCCGCCGCC } \\
\text { GCCAT }\end{array}$ \\
\hline $\begin{array}{l}\text { GST-MST27-TASK3-C15 } \\
\text { WT FP } \\
\text { 5'[Clal - Xhol]3' }\end{array}$ & $\begin{array}{l}\text { Phosphorylated oligonucleotide } \\
\text { that encodes the last } 15 \text { amino } \\
\text { acids of the TASK3 C-terminus, } \\
\text { used for annealing. }\end{array}$ & $\begin{array}{l}\text { CGATGGCGGCGGCGGC } \\
\text { TCGGGCGGCGGCGGCT } \\
\text { CTAGCTTTACCGACCAC } \\
\text { CAGAGGCTGATGAAAC } \\
\text { GCCGGAAGTCCGTTTAA } \\
\text { C }\end{array}$ \\
\hline $\begin{array}{l}\text { GST-MST27-TASK3-C15 } \\
\text { WT RP } \\
\text { 5'[Xhol - Clal]3' }\end{array}$ & $\begin{array}{l}\text { Phosphorylated oligonucleotide } \\
\text { that encodes the last } 15 \text { amino } \\
\text { acids of the TASK3 C-terminus, } \\
\text { used for annealing. }\end{array}$ & $\begin{array}{l}\text { TCGAGTTAAACGGACTT } \\
\text { CCGGCGTTTCATCAGCC } \\
\text { TCTGGTGGTCGGTAAAG } \\
\text { CTAGAGCCGCCGCCGC } \\
\text { CCGAGCCGCCGCCGCC } \\
\text { AT }\end{array}$ \\
\hline
\end{tabular}




\section{Material and Methods}

Table 4: Antibodies employed in this study.

\begin{tabular}{|c|c|c|c|c|c|c|}
\hline Antigen & Name & Source & Cat.No. & Lot No. & $\begin{array}{c}\text { Dilution } \\
\text { and } \\
\text { Technique }\end{array}$ & Type \\
\hline $\begin{array}{l}\text { CD8- } \\
\text { alpha }\end{array}$ & $(H-160)$ & $\begin{array}{c}\text { Santa Cruz } \\
\text { Biotech. }\end{array}$ & $\begin{array}{l}\text { SC- } \\
7188\end{array}$ & \#E2213 & $\begin{array}{c}\text { 1:1000 } \\
\text { Western } \\
\text { blotting }\end{array}$ & Polyclonal \\
\hline $\begin{array}{l}\text { CD8- } \\
\text { alpha }\end{array}$ & (UCHT-4) & Sigma & C7423 & \#044M4833 & $\begin{array}{c}5 \mu \mathrm{l} / 10^{6} \\
\text { cells } \\
\text { Flow } \\
\text { cytometry }\end{array}$ & Monoclonal \\
\hline $\begin{array}{c}\text { Phospho- } \\
\text { PKA } \\
\text { subst. }\end{array}$ & (100G7E) & $\begin{array}{l}\text { Cell Signaling } \\
\text { Technologies }\end{array}$ & \#9623 & 0016 & SPR & Polyclonal \\
\hline Caot & $(T-270)$ & Anne Spang & & & $\begin{array}{c}\text { 1:1000 } \\
\text { Western } \\
\text { blotting }\end{array}$ & Polyclonal \\
\hline GST & $\begin{array}{c}\text { Anti-GST } \\
\text { pAB }\end{array}$ & Carl Roth & 3998.1 & 2950 & $\begin{array}{c}200 \mathrm{nM} \\
\mathrm{SPR}\end{array}$ & Polyclonal \\
\hline
\end{tabular}




\section{Results}

\section{Quantitative characterization of 14-3-3 binding to TASK-derived C- terminal peptides}

Current insight into 14-3-3 binding to a trafficking control region at the distal Cterminus of TASK-1 and TASK-3 is almost exclusively qualitative $(11,17-19)$. It is well understood that a prerequisite of 14-3-3 binding, is phosphorylation of a serine present at the distal C-terminus of TASK-1 (S393) and TASK-3 (S373). Also well established is that co-expression of the channel protein and 14-3-3 increases the relative amount of protein expressed at the cell surface, indicating the necessity of 14-3-3 binding to overcome ER retention. However, how binding affinities between different isoforms (seven isoforms are known in mammals, denoted with Greek letters $\beta, \gamma, \varepsilon, \zeta, \eta, \tau, \sigma)$ and different substrates, such as TASK-1 and TASK-3, compare is unknown. In 2013 the first insight into molecular details of 14-3-3 binding to this trafficking control region was gained by Andes et al. (2013) determining the binding affinity of 14-3-3 $\sigma$ to a TASK-3 derived hexapeptide with a dissociation constant of $\mathrm{K}_{\mathrm{D}}=4.1 \pm 0.8 \mu \mathrm{M}(107)$.

To assess the binding parameters of all seven 14-3-3 isoforms to a large variety of TASK-derived client peptides systematically, fluorescence polarization titration (FP) was employed. 14-3-3 clients comprising the last 15 amino acids of either TASK-3, various TASK-3 derived mutants and TASK-1derived peptides are listed in Table 5. Employing a solution-based method and synthetic peptides appeared to be advantageous to determine 14-3-3 binding parameters for differentially phosphorylated TASK-1 derived peptides (TASK-1 presents two adjacent serine residues at its distal C-terminus $\left(\mathrm{KRRS}^{392} \mathrm{~S}^{393} \mathrm{~V}\right)$, compared to TASK-3 with one Lysine residue preceding the conserved Serine residue of the mode III 14-3-3 binding motif $\left.\left(\mathrm{KRRK}^{372} \mathrm{~S}^{373} \mathrm{~V}\right)\right)$. Furthermore this method appeared to be particularly useful to investigate binding of one fluorescent TASK-derived peptide to one binding groove of 14-3-3 in solution. 


\section{Results}

\begin{tabular}{ll} 
Peptide name & Amino acid sequence \\
\hline TASK-3 WT & Fc-CSFTDHQRLMKRRKSV-COOH \\
TASK-3 WT pS373 & Fc-CSFTDHQRLMKRRKpSV-COOH \\
TASK-3 V374 & Fc-CSFTDHQRLMKRRKpS-COOH \\
TASK-3 S373A & Fc-CSFTDHQRLMKRRKAV-COOH \\
TASK-3 K369A pS373 & Fc-CSFTDHQRLMARRKpSV-COOH \\
& \\
TASK-1 WT & Fc-CSLSTFRGLMKRRSSV-COOH \\
TASK-1 WT pS392 & Fc-CSLSTFRGLMKRRpSSV-COOH \\
TASK-1 WT pS393 & Fc-CSLSTFRGLMKRRSpSV-COOH \\
TASK-1 WT pS392_pS393 & Fc-CSLSTFRGLMKRRpSpSV-COOH \\
TASK-1 S392A_S393A & Fc-CSLSTFRGLMKRRAAV-COOH \\
TASK-1 pS393 $\Delta$ V394 & Fc-CSLSTFRGLMKRRSpS-COOH
\end{tabular}

Table 5: Peptides employed in Fluorescence polarization titration (FP) experiments. Trafficking control motifs and important residues are highlighted in different colors. Green: ER retention/retrieval motif; Red: phosphorylated Serine residues, mode III 14-3-3 binding motif; Blue: Alanine mutations disrupting either the previously mentioned ER retention/retrieval motif, or 14-3-3 binding.

\section{4-3-3 proteins bind the TASK-3 C-terminus phosphorylation dependent and with high affinity}

First I assessed binding of all seven mammalian 14-3-3 isoforms to peptides comprising the last 15 amino acids of TASK-3. Peptides employed were: an unphosphorylated version of the TASK-3 C-terminus (TASK-3 WT), a phosphorylated version of the TASK-3 WT (TASK-3 WT pS373) and a 14-3-3 binding deficient version of the TASK-3 C-terminus (TASK-3 S373A). All seven mammalian 14-3-3 isoforms were affinity purified as fusions of the maltose binding protein (MBP). After cleavage of the MBP protein-tag, by Factor $\mathrm{Xa}$, dimeric 14-3-3 proteins were obtained by size exclusion chromatography. 
Binding parameters of all seven 14-3-3 isoforms to the TASK-3 WT Cterminus were determined, titrating $10 \mathrm{nM}$ of the fluorescent peptide with 14-33 concentrations in a range of $1 \mathrm{nM}$ to $120 \mu \mathrm{M}$ (Figure 8). The FP data was analyzed applying a sigmoidal dose-response curve fit (monophasic). Binding parameters determined for TASK-3 WT pS373 ranged from 110 $110 \mathrm{nM}$ (14-3$3 \eta$, Figure 8, E) to $3,600 \pm 400 \mathrm{nM}(14-3-3 \sigma$, Figure 8, G). Titration of the unphosphorylated TASK-3 WT peptide with 14-3-3 proteins did not yield significant changes in fluorescence polarization. Similar observations were made, titrating TASK-3 S373A with 14-3-3 proteins. Calculated equilibrium binding constants are listed in Table 6.

$F P: K_{D}[n M]$

\begin{tabular}{|c|c|c|c|c|c|}
\hline \multirow[b]{2}{*}{$14-3-3 \beta$} & \multirow{2}{*}{$\begin{array}{c}\text { TASK-3 WT } \\
\text { n.b. }\end{array}$} & \multicolumn{3}{|c|}{ TASK-3 WT pS373 } & \multirow{2}{*}{$\begin{array}{c}\text { TASK-3 S373A } \\
\text { n.b. }\end{array}$} \\
\hline & & 370 & \pm & 40 & \\
\hline $14-3-3 y$ & n.b. & 150 & \pm & 20 & n.b. \\
\hline $14-3-3 \varepsilon$ & n.b. & 800 & \pm & 80 & n.b. \\
\hline $14-3-3 \zeta$ & n.b. & 1,000 & \pm & 60 & n.b. \\
\hline $14-3-3 n$ & n.b. & 110 & \pm & 10 & n.b. \\
\hline $14-3-3 T$ & n.b. & 220 & \pm & 20 & n.b. \\
\hline $14-3-3 \sigma$ & n.b. & 3,600 & \pm & 400 & n.b. \\
\hline
\end{tabular}

Table 6: Equilibrium binding constants for respective interaction pairs of TASK-3 derived peptides and different 14-3-3 isoforms. n.b.: no binding observed. Values are displayed as average, error depicted as standard error of the mean (s.e.m.).

Taken together these finding demonstrate that $14-3-3$ binding to the TASK-3 WT C-terminus depends on the phosphorylation state of S373, comparing interaction experiments performed with phosphorylated (TASK-3 WT pS373), unphosphorylated (TASK-3 WT) or 14-3-3 binding deficient (TASK-3 S373A) TASK-3-derived C-terminal peptides. It could furthermore be shown, that 143-3 proteins bind the phosphorylated TASK-3 C-terminus with high affinity in an isoform specific manner (Figure 8, Table 6). 


\section{Results}

A

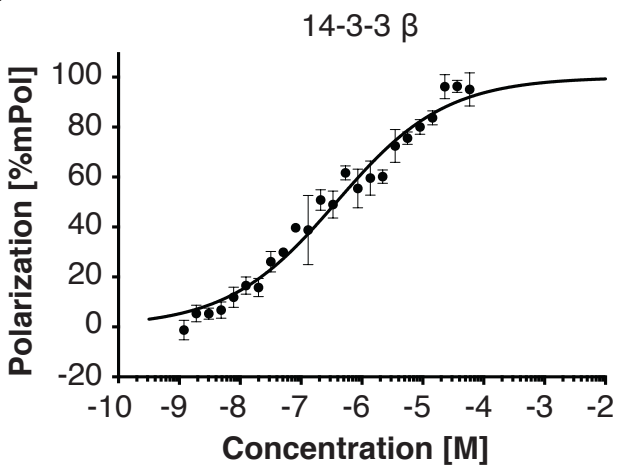

C

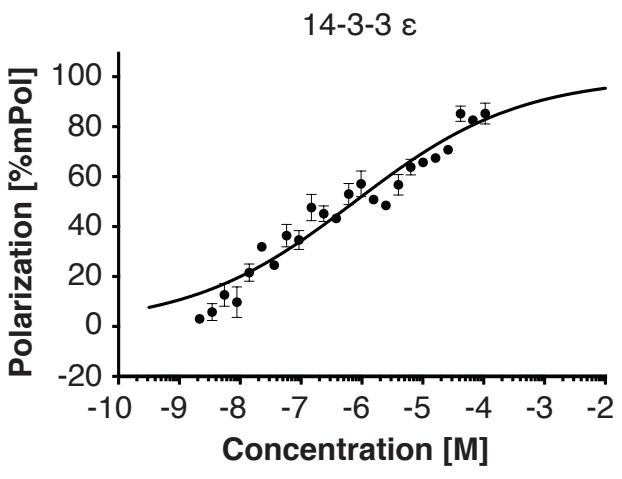

$\mathbf{E}$

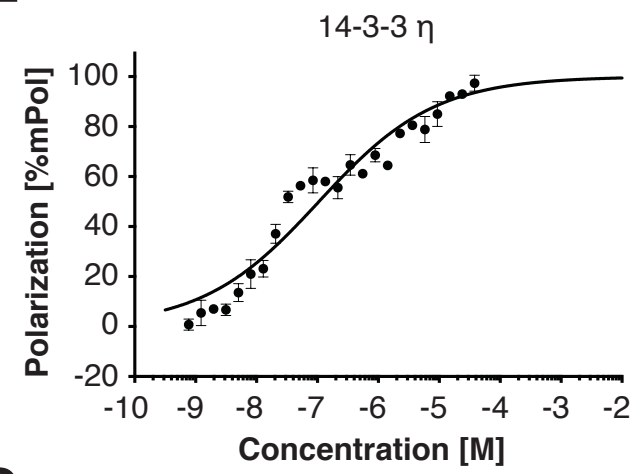

G

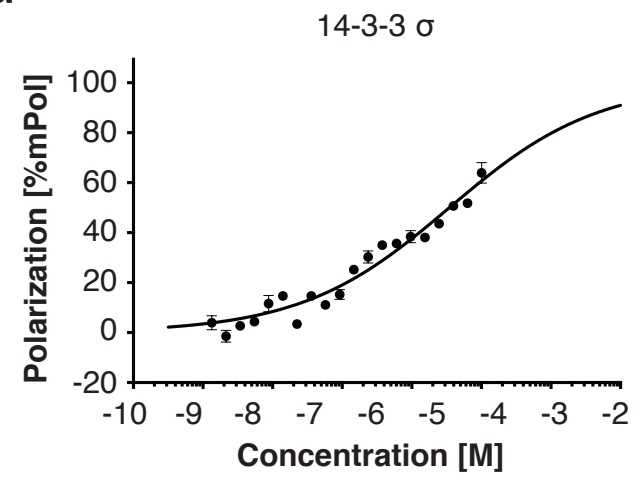

B

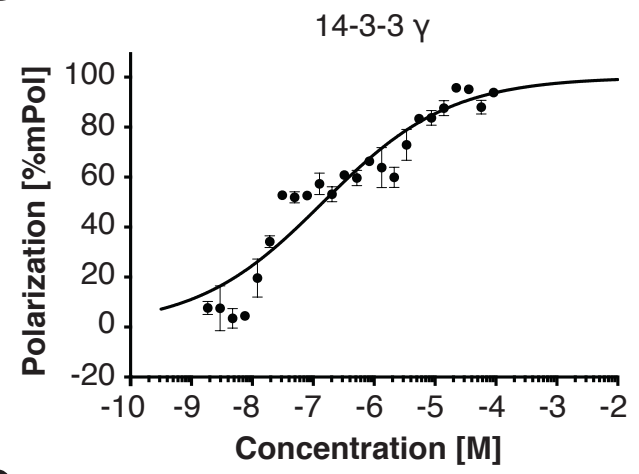

D

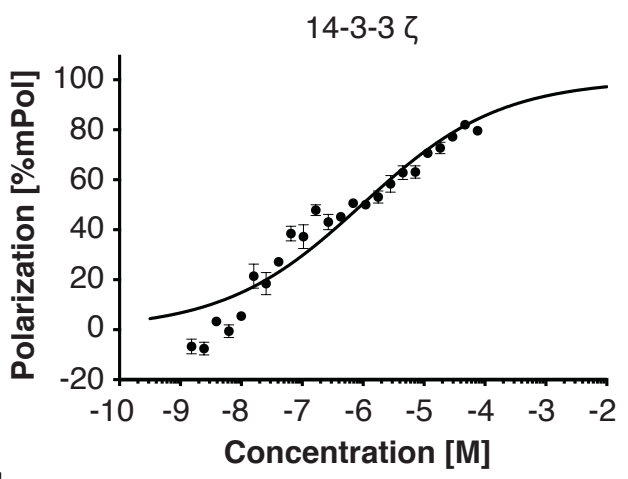

$\mathbf{F}$

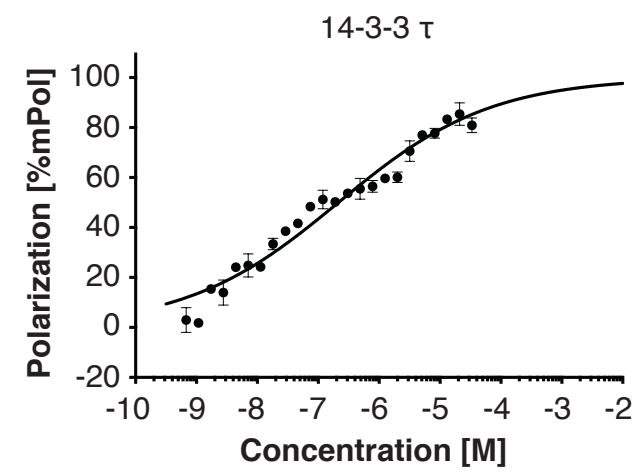

Figure 8: Isoform specific binding of 14-3-3 proteins to the phosphorylated TASK3 WT C-terminus. A to G illustrate equilibrium binding isotherms obtained for interactions measured between 14-3-3 proteins and peptides comprising the distal (CT15), phosphorylated TASK3 C-terminus (Respective interaction pairs were 


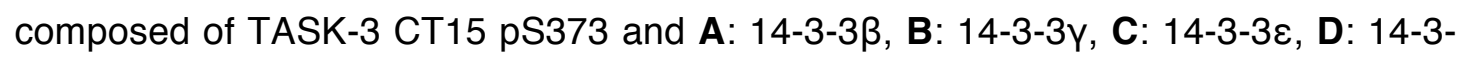

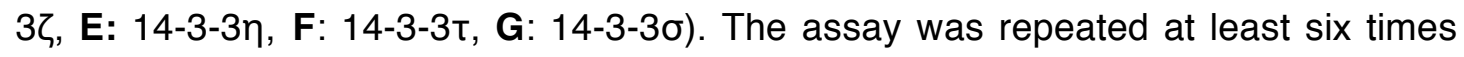
with two batches of independently purified protein. Error bars depict standard error of the mean (s.e.m.).

\section{Introducing a K369A mutation into the TASK3 C-terminus does not disrupt 14-3-3 binding}

We further investigated binding of all seven mammalian 14-3-3 isoforms to a COPI-binding deficient version of the TASK3 C-terminus, TASK3 K369A. Mutation of the non-canonical ER retention/retrieval motif present in TASK-3, $\mathrm{K}^{369} \mathrm{R}^{370} \mathrm{R}^{371}$ (3, 11, 17-19, 113), disrupts COPI binding resulting in elevated cell surface expression of the channel. The disruption of COPI binding also removes the necessity of phosphorylation dependent 14-3-3 binding to overcome ER-retention (11), allowing COPI- and 14-3-3-independent cell surface expression of the channel.

Affinity purified and proteolytically processed and gel-filtrated 14-3-3 proteins were titrated with $10 \mathrm{nM}$ of the fluorescent peptide. Experiments were carried out and analyzed as described above. Calculated binding affinities ranged

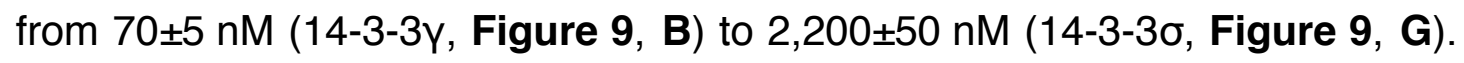
A comparison of values obtained for respective interaction pairs of TASK3 WT pS373, TASK3 K369A pS373 with different 14-3-3 isoforms is illustrated in Table 7.

Comparing absolute values, 14-3-3 binding affinities for TASK-3 K369A pS373 were increased by up to 2-fold for 14-3-3y and 14-3-3ろ, compared to binding affinities determined for TASK-3 WT pS373. In general, an increase in binding affinity was observed for all 14-3-3 isoforms, except 14-3-3 $\tau$, for which a reduction in binding affinity by 2 -fold was observed (see Table 7 for a direct comparison). 


\section{Results}

A

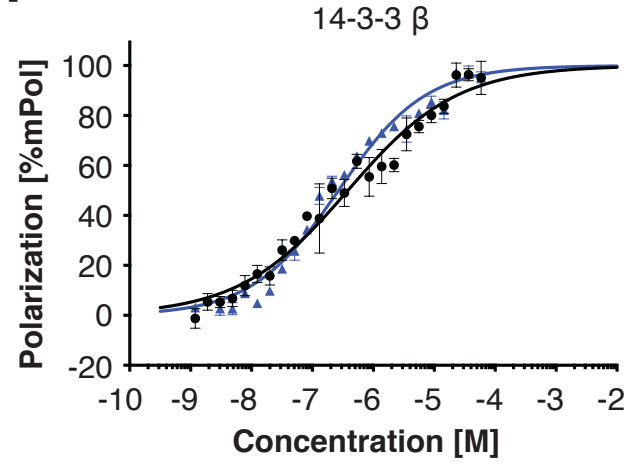

C

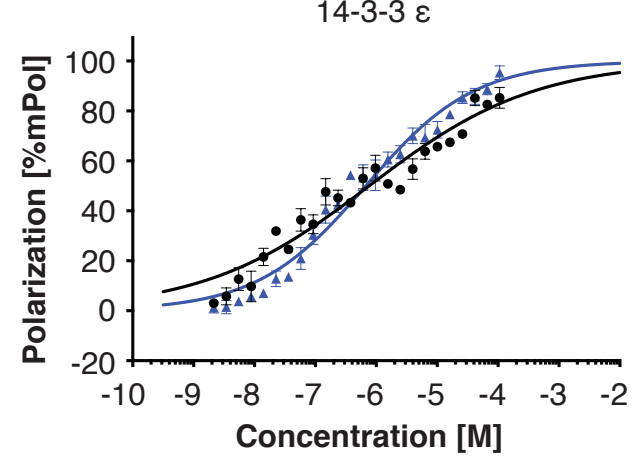

$\mathbf{E}$

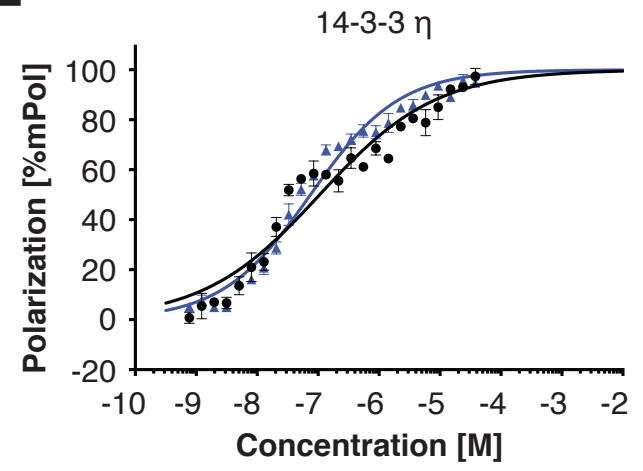

G

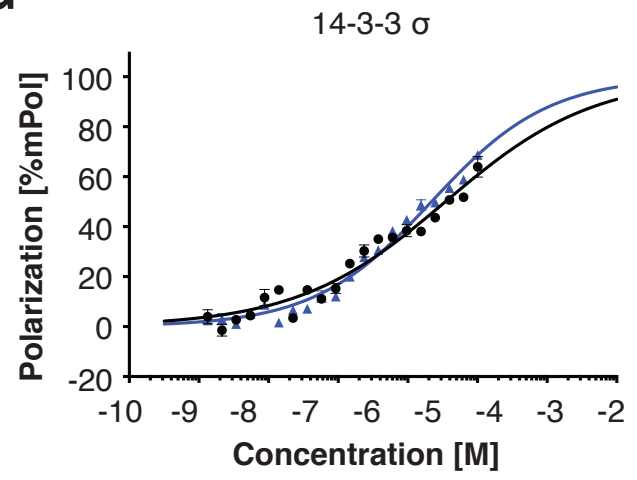

B

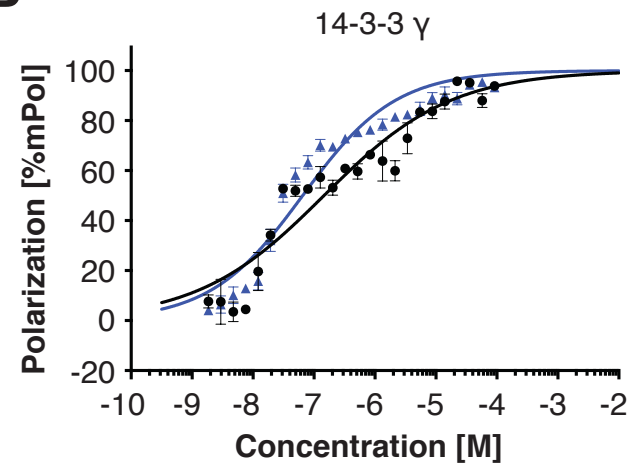

D

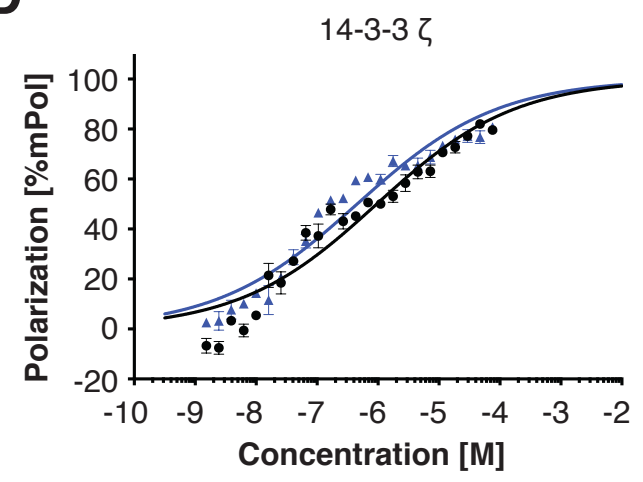

$\mathbf{F}$

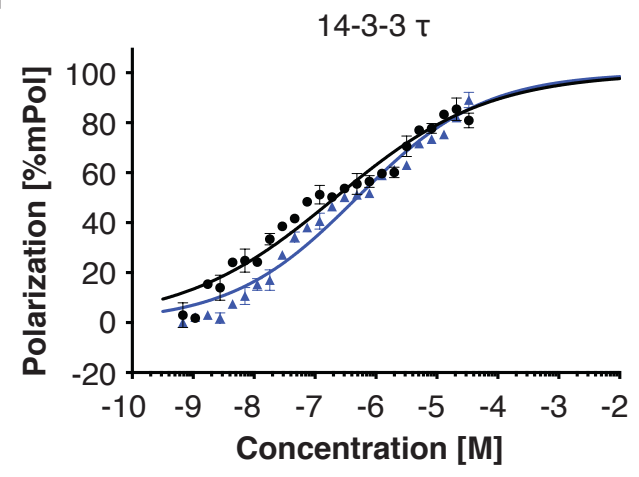

- TASK3 pS373

- TASK3 K369A pS373

Figure 9: Mutation of K369 to Alanine does not interfere with 14-3-3 binding. A to G illustrate an overlay of equilibrium binding isotherms obtained for respective interaction pairs of TASK-3 K369A pS373 (blue line) and TASK-3 WT pS373 (black line) with different 14-3-3 isoforms. The assay was repeated at least six times with 
Results

two batches of independently purified protein. Error bars depict standard error of the mean (s.e.m.).

I conclude that 14-3-3 binding is not impaired upon replacement of K369 by Alanine. Hence reporter proteins containing such a mutation should maintain 14-3-3 binding properties once phosphorylated.

$$
F P: K_{D}[n M]
$$

Change

TASK-3 WT pS373

TASK-3 K369A pS373

[fold]

14-3-3 $\beta$

14-3-3y

14-3-3 $\varepsilon$

$370 \pm 40$

$150 \pm 20$

$270 \pm 10$

1.4

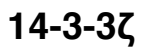

14-3-3n

14-3-3T

14-3-3o

$800 \pm 80$

$70 \pm 5$

$700 \pm 200$

$460 \pm 20$

$80 \pm 3$

1.4

$490 \pm 30$

$-2.0$

$220 \pm 20$

$2200 \pm 50$

1.6

Table 7: Affinity binding constants determined for respective interaction pairs of TASK-3 K369A pS373 and different mammalian 14-3-3 isoforms. Values are displayed as the average of six measurements. Error depicts standard error of the mean (s.e.m.).

\section{Deletion of V374 does not abolish 14-3-3 binding}

Based on the current literature $(11,18)$ deletion of a C-terminal valine, which is part of a mode III 14-3-3 binding motif in TASK3, abolishes 14-3-3 binding. Furthermore this valine residue appears to be required to bind 14-3-3 proteins with high affinity, facilitating channel release from ER retention (11, 17-19, 113-115). We tested this hypothesis and assessed 14-3-3 binding to peptides comprising the last 14 amino acids of TASK-3, lacking the penultimate Valine residue. Peptides used were phosphorylated at S373 to enable 


\section{Results}

phosphorylation-dependent binding of 14-3-3 proteins. Experiments were carried out and analyzed as described above.

Interestingly, we found that almost all 14-3-3 isoforms, except 14-3-30, bound the TASK-3 pS373 $\triangle$ V374 peptide, with binding affinities varying from $1.1 \pm 0.1 \mu \mathrm{M}(14-3-3 \gamma$, Figure 10, B) to $18.0 \pm 5.0 \mu \mathrm{M}(14-3-3 \varepsilon$, Figure 10, B).

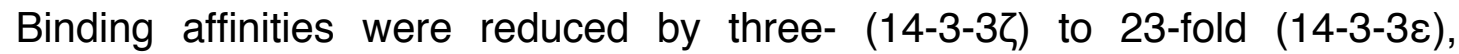
compared to affinities determined for TASK-3 WT pS373 (Table 8).

FP: $K_{\mathrm{D}}[\mathrm{nM}]$

\section{TASK-3 WT pS373}

14-3-3 $\beta$

14-3-3y

14-3-3ع

14-3-3द

14-3-3n

14-3-3T

14-3-3o

$\begin{array}{rrr}370 & \pm & 40 \\ 150 & \pm & 20 \\ 800 & \pm & 80 \\ 1,000 & \pm & 60 \\ 110 & \pm & 10 \\ 220 & \pm & 20 \\ 3,600 & \pm & 400\end{array}$

TASK-3 $\Delta$ V374 pS373

$1,200 \pm 100$

$1,100 \pm 140$

$18,000 \pm 4,800$

$3,300 \pm 300$

$1,600 \pm 140$

$2,400 \pm 100$

n.b. \pm n.b.

\section{Change}

\section{[fold]}

10.9

Table 8: Affinity binding constants determined for respective interaction pairs of TASK-3 WT pS373, TASK-3 pS373 $\triangle$ V374 and different mammalian 14-3-3 isoforms. Values are displayed as the average of six measurements. Error depicts standard error of the mean (s.e.m.). n.b.: no binding observed.

Comparing affinities, we conclude, that 14-3-3 binding to the truncated TASK3 C-terminus (TASK-3 pS373 $\Delta$ V374) is changed significantly, but not entirely lost upon deletion of the penultimate Valine. A recent crystal structure also revealed that V374 forms only one hydrogen bond with a lysine residue in 14$3-3 \sigma$, thereby not substantially contributing to binding affinities observed. Since all experiments were carried out with fully phosphorylated peptides, further information about the phosphorylation state of the truncated TASK-3 channel/reporter protein in vivo is required. Hence the lack of cell surface 
expression reported in the literature cannot fully be explained by reduced 143-3 binding affinities.

A

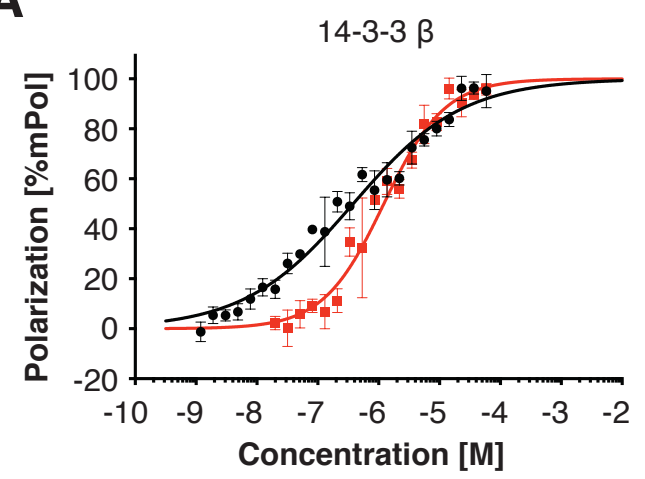

C

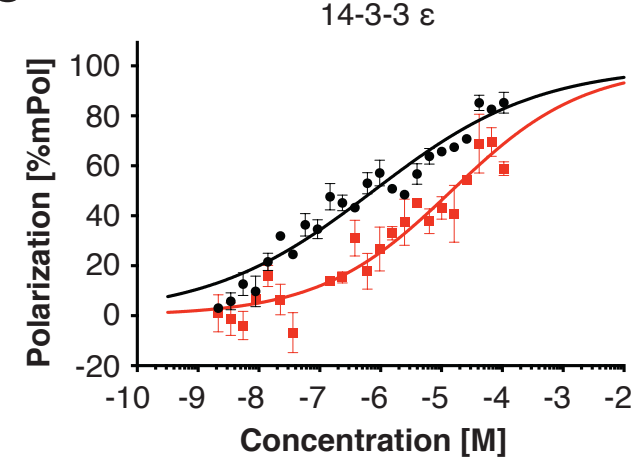

E

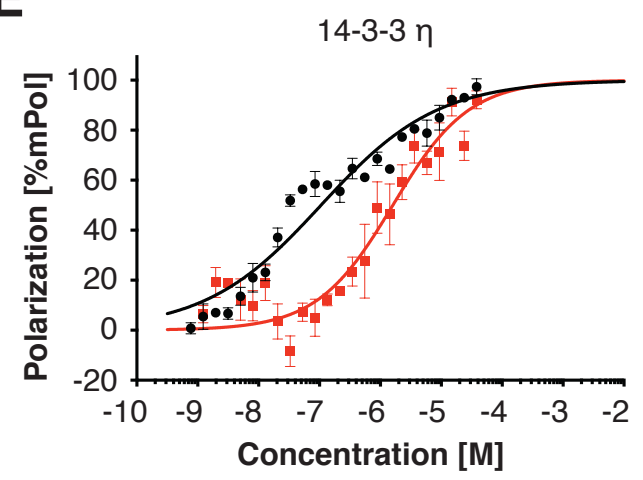

B

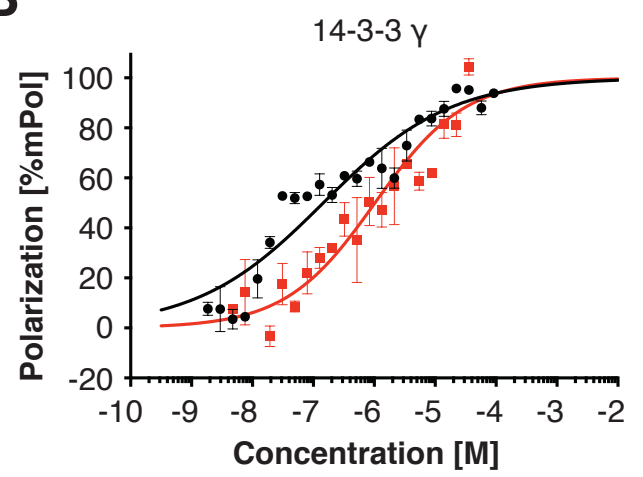

D

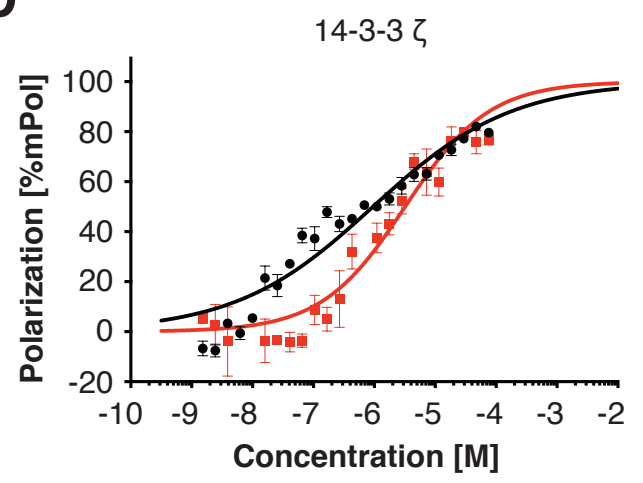

$\mathbf{F}$

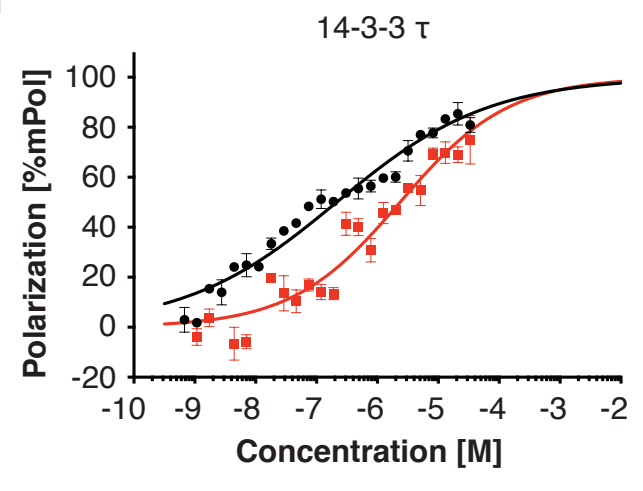

TASK3 pS373

- TASK3 AV374 pS373

Figure 10: Truncation of the TASK-3 C-terminus does not abolish 14-3-3 binding. A to $\mathbf{F}$ illustrate equilibrium binding isotherms for respective interaction pairs of TASK-3 pS373 $\Delta$ V374 (red line) and TASK-3 WT pS373 (black line) with different 14-3-3 isoforms. No interactions between 14-3-3 $\sigma$ and TASK-3 $\Delta \mathrm{V} 374$ pS373 were observed (isotherm not shown). The assay was repeated at least six times with two 


\section{Results}

batches of independently purified protein. Error bars depict standard error of the mean (s.e.m.).

\section{Modulation of 14-3-3 binding by differential phosphorylation of the client protein}

Having systematically characterized interactions between 14-3-3 proteins and TASK-3 derived C-terminal peptides, I was further interested in how binding parameters compare between two different client proteins, such as TASK-3 and TASK-1. We utilized the same experimental approach as described for TASK-3 and employed fluorescently labeled peptides comprised of the last 15 amino acids of the distal TASK-1 C-terminus.

There are notable differences in the residues flanking the COPI retentionretrieval motif and the mode III 14-3-3 binding motif in TASK-1 and TASK-3. TASK-1 features a second serine residue (S392) that can potentially be phosphorylated and that precedes the conserved serine (S393) of the mode III 14-3-3 binding motif. This second serine is absent in TASK-3, where the corresponding residue flanking both motifs is lysine (K372). To address the presence of this second Serine residue in TASK-1 and the functional consequence of it being phosphorylated on 14-3-3 binding, individually phosphorylated C-terminal peptides, TASK-1 WT pS392 and TASK-1 WT pS393 as well as a double phosphorylated peptide TASK-1 WT pS392pS393 were employed. Additionally, I characterized interactions between all 14-3-3 isoforms and a truncated version of the TASK-1 C-terminus, TASK-1 pS393 $\triangle$ V394. A 14-3-3 binding deficient mutant of the TASK-1 C-terminus, TASK-1 S392/393A, and the unphosphorylated C-terminal TASK-1 peptide, TASK-1 WT were used as controls, which essentially did not bind 14-3-3. A list of all peptides employed in Fluorescence Polarization titration assays can be found in Table 5. 
Results

Binding affinities of 14-3-3 proteins for the TASK-1 WT C-terminus are significantly lower than for the TASK-3 WT C-terminus

I first determined the binding parameters for all seven mammalian 14-3-3 isoforms to TASK-1 WT pS393, in which the conserved Serine residue of the mode III 14-3-3 binding motif was phosphorylated (3, 11, 17-19, 107, 113, 116). Experiments were carried out and the obtained data was analyzed as described for TASK3 derived C-terminal peptides. Control experiments were performed employing an unphosphorylated TASK-1 WT peptide, as well as a 14-3-3 binding deficient mutant of the TASK-1 C-terminus (TASK-1 S392/393A). 14-3-3 proteins bound the TASK-1 WT pS393 peptide with affinities ranging from $7.5 \pm 0.1 \mu \mathrm{M}(14-3-3 \beta$, Figure $11, \mathbf{A})$ to $49.4 \pm 2.0 \mu \mathrm{M}$ (143-3t, Figure 11, E). Compared to TASK-3 WT pS373, affinities were reduced

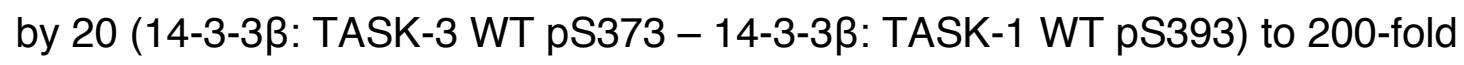
(14-3-3t: TASK-3 WT pS373 - 14-3-3t: TASK-1 WT pS393), comparing individual isoforms (Table 9). We did not observe 14-3-3 binding to either TASK-1 WT (not phosphorylated), or TASK-1 S392/393A.

\section{FP: $\mathrm{K}_{\mathrm{D}}[\mu \mathrm{M}]$}

TASK-1 WT TASK-1 WT pS393

TASK-1 S392/393A

\begin{tabular}{|c|c|c|c|c|c|}
\hline $14-3-3 \beta$ & n.b. & 7.5 & \pm & 0.1 & n.b. \\
\hline $14-3-3 y$ & n.b. & 9.8 & \pm & 0.6 & n.b. \\
\hline $14-3-3 \varepsilon$ & n.b. & 29.0 & \pm & 1.0 & n.b. \\
\hline $14-3-3 \zeta$ & n.b. & 23.0 & \pm & 1.4 & ). \\
\hline $14-3-3 n$ & n.b. & 9.2 & \pm & 0.1 & . \\
\hline 14-3-3т & n.b. & 49.4 & \pm & 2.0 & n.b. \\
\hline $14-3-3 \sigma$ & n.b. & 36.0 & \pm & 2.9 & n.b. \\
\hline
\end{tabular}

Table 9: Affinity binding constants determined for respective interaction pairs of TASK-1 WT, TASK-1 WT pS393, TASK-1 S392/393A and different mammalian 14-33 isoforms. Values are display as the average of six experiments. The error depicts standard error of the mean (s.e.m.). n.b.: no binding observed. 


\section{Results}

Taken together my findings illustrate, that 14-3-3 proteins bind the phosphorylated TASK-1 WT C-terminus in an isoform specific manner with binding affinities substantially reduced (low $\mu \mathrm{M}$ range), compared to affinities determined for TASK-3 WT pS373 (low nM range). I further demonstrate, that 14-3-3 binding dependents on the phosphorylation state of S393, compare experiments performed with phosphorylated (TASK-1 WT pS393), unphosphorylated (TASK-1 WT) and 14-3-3 binding deficient (TASK-1 S392/393A) TASK-1 derived C-terminal peptides.

\section{Truncation of the TASK-1 C-terminus disrupts 14-3-3 binding}

I further investigated binding of all seven mammalian 14-3-3 isoforms to the truncated TASK-1 C-terminus, TASK-1 pS393 $\Delta$ V394. Although phosphorylated at S393, 14-3-3 binding to the truncated TASK-1 C-terminus was no longer detected. These findings are in agreement with current literature $(11,18)$. A comparison between TASK-3 pS373 $\Delta$ V374 and TASK-1 pS393 $\Delta$ V394 illustrates the impact of different residues presented by each Cterminal peptide on 14-3-3 binding. Whereas TASK-3 presents a lysine (K372) residue that possibly forms hydrogen bonds with the side chains present in the inner side of the 14-3-3 binding cleft, TASK-1 C-terminal peptides present a second serine residue (S392) that cannot interact in the same way and hence affects the 14-3-3 binding properties (Table 9, Figure 11). 
A

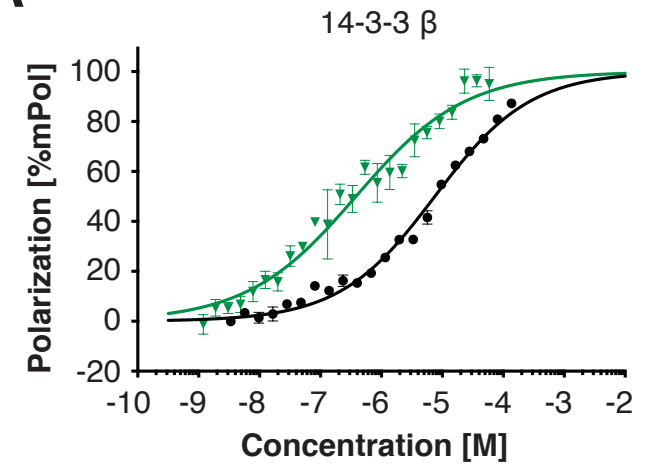

C

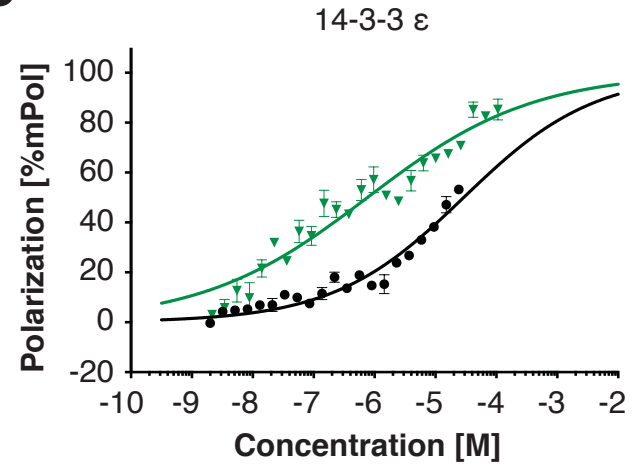

$\mathbf{E}$

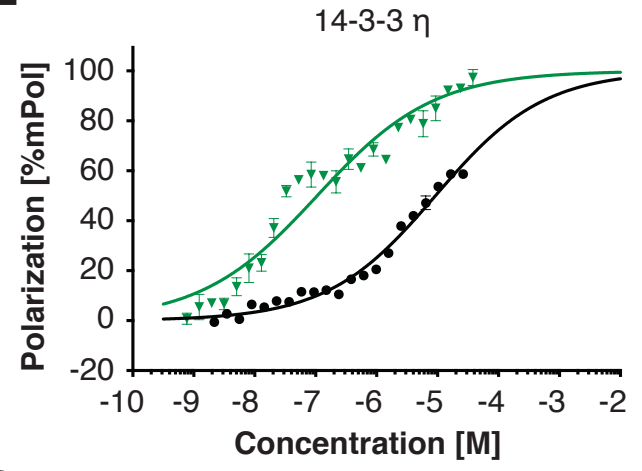

G

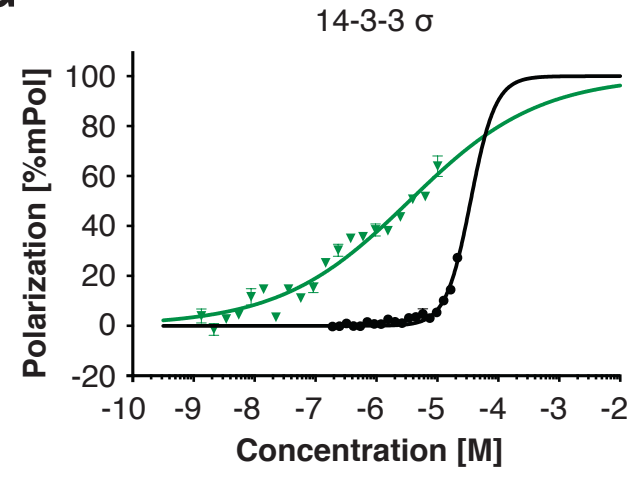

B

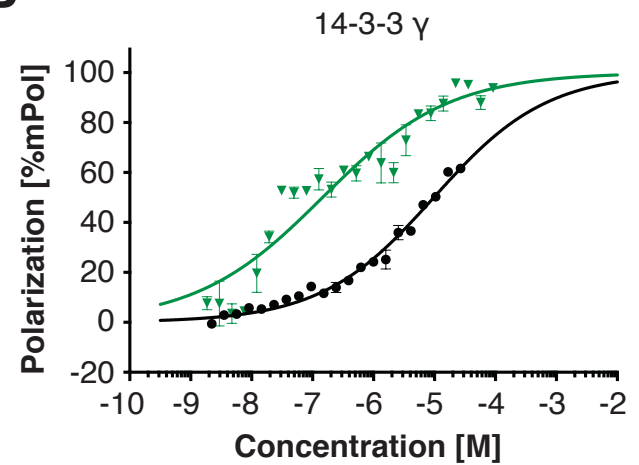

D

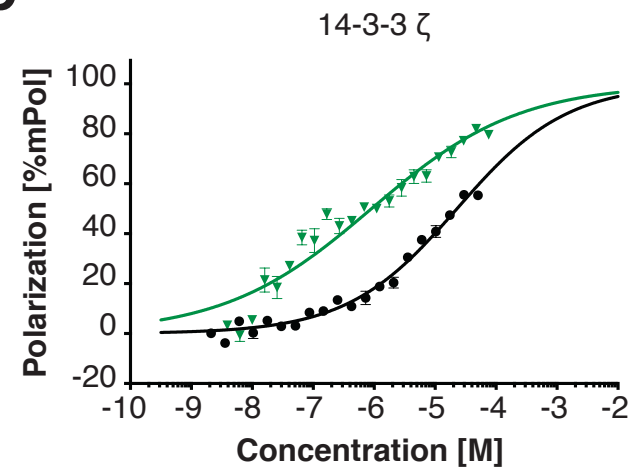

$\mathbf{F}$

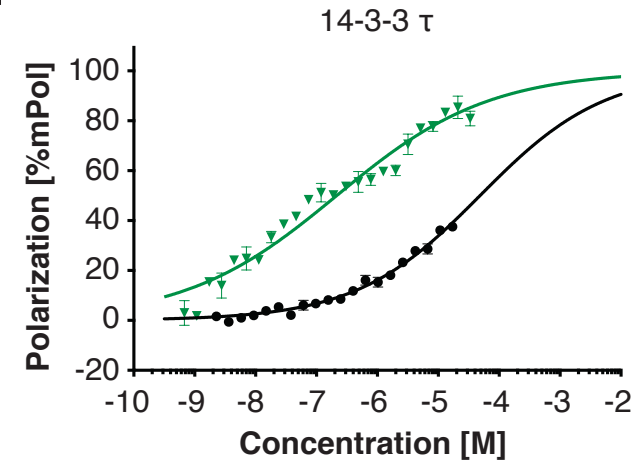

Figure 11: Binding affinities of 14-3-3 proteins to TASK-1 pS393 are reduced by several orders of magnitude compared to affinities determined for TASK-3 WT pS373. A to $\mathbf{G}$ illustrate equilibrium binding isotherms obtained for respective interaction pairs of TASK-3 WT pS373 (green line) and TASK-1 WT pS393 (black 


\section{Results}

line) with different 14-3-3 isoforms. The assay was repeated at least six times with two batches of independently purified protein. Error bars depict standard error of the mean (s.e.m.).

\section{Phosphorylation of S392 inhibits 14-3-3 binding}

To further evaluate the consequence of phosphorylation of the second Serine residue (S392), present in the TASK-1 C-terminus, on 14-3-3 binding, peptides phosphorylated at S392, TASK-1 WT pS392, and double phosphorylated peptides, TASK-1 pS392pS393 were employed. FP experiments were carried out and data was analyzed as described for TASK-3 derived C-terminal peptides. Theoretically phosphorylation of S392 could create another high/moderate affinity 14-3-3 binding site, which would fulfill the criteria of a mode III 14-3-3 binding motifs (-R-X-pS/T- $\left.\mathrm{X}_{1-2}-\mathrm{COOH}(15)\right)$. We tested this possibility and determined equilibrium binding parameters for all seven 14-3-3 isoforms binding to TASK-1 WT pS392 (Figure 12). Interestingly binding affinities determined were significantly lower than for TASK-1 WT pS393 and TASK-3 WT pS373, varying from 33.1 $\pm 0.7 \mu \mathrm{M}$ (14-3$3 \beta$, Figure 12, A, Table 10) to $373.6 \pm 33.2 \mu \mathrm{M}(14-3-3 \varepsilon$, Figure 12, C, Table 10). Compared to TASK-1 WT pS393 affinities were reduced by five (14-3-3 TASK-1 WT pS393 - TASK-1 WT pS392) to 13-fold (14-3-3ع: TASK1 WT pS393 - TASK-1 WT pS392). 
FP: $K_{D}[n M]$

\section{TASK-3 WT pS373}

$\begin{array}{lrlr}14-3-3 \beta & 370 & \pm & 40 \\ 14-3-3 \gamma & 150 & \pm & 20 \\ 14-3-3 \varepsilon & 800 & \pm & 80 \\ 14-3-3 \zeta & 1,000 & \pm & 60 \\ 14-3-3 \eta & 110 & \pm & 10 \\ 14-3-3 т & 220 & \pm & 20 \\ 14-3-3 \sigma & 3,600 & \pm & 400\end{array}$

FP: $\mathrm{K}_{\mathrm{D}}[\mu \mathrm{M}]$

\begin{tabular}{rccrcr}
\hline TASK-1 WT & pS393 & \multicolumn{3}{c}{ TASK-1 WT } & pS392 \\
7.5 & \pm & 0.1 & 33.1 & \pm & 0.7 \\
9.8 & \pm & 0.6 & 51.4 & \pm & 2.7 \\
29.0 & \pm & 1.0 & 373.6 & \pm & 33.2 \\
23.0 & \pm & 1.4 & 133.0 & \pm & 1.8 \\
9.2 & \pm & 0.1 & 81.5 & \pm & 7.8 \\
49.4 & \pm & 2.0 & 123.2 & \pm & 9.0 \\
36.0 & \pm & 2.9 & 58.8 & \pm & 5.3
\end{tabular}

Table 10: Affinity binding constants determined for respective interaction pairs of TASK-1 WT pS393, TASK-1 WT pS392 and different mammalian 14-3-3 isoforms. For comparison binding affinities of 14-3-3 proteins to TASK-3 WT pS373 are included. Values are display as the average of six experiments. Error depicts standard error of the mean (s.e.m.).

These findings confirmed that phosphorylation of S392 does not compensate for phosphorylation of S393 by creating another high/moderate affinity 14-3-3 binding site. On the contrary, phosphorylation of S392 seems to exert an inhibitory effect on 14-3-3 binding. I also predict a potential regulatory function of S392 in protein trafficking, implying an even more complex signal transduction cascade regulating TASK-1 anterograde protein trafficking. 


\section{Results}

A

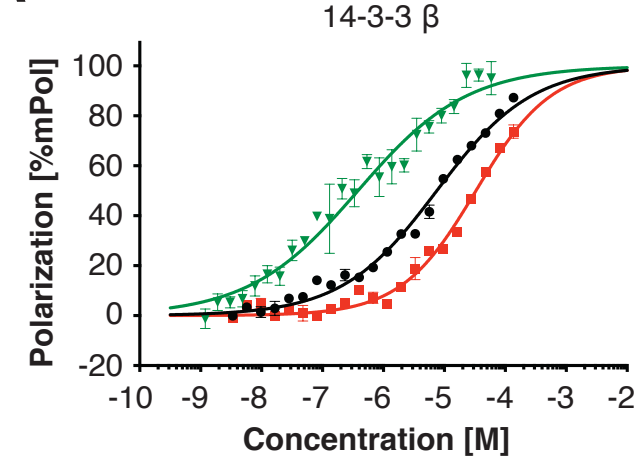

C

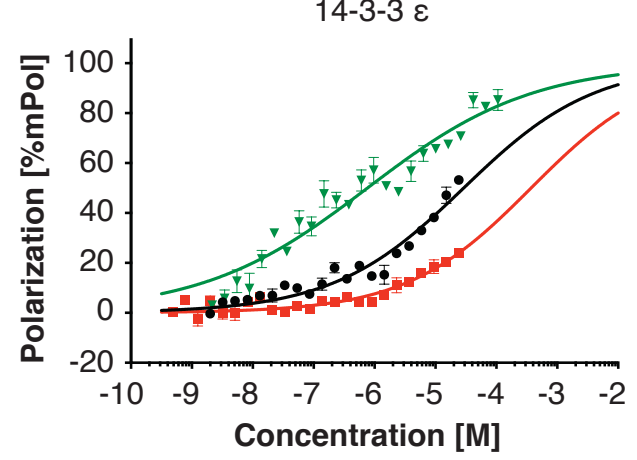

E

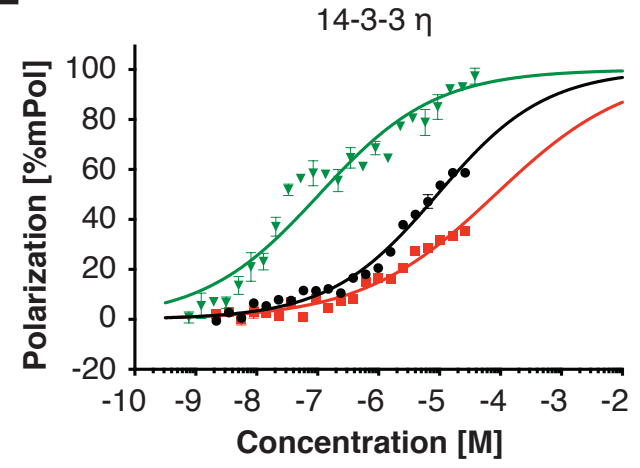

G

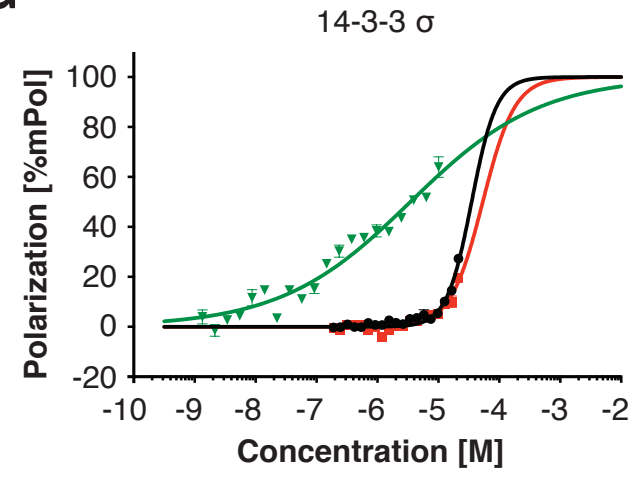

B

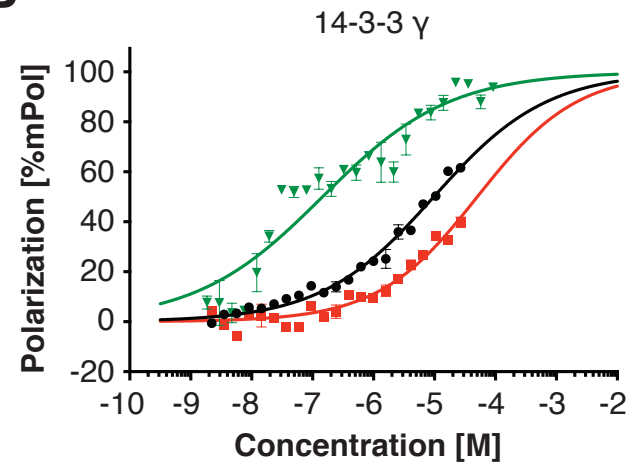

D

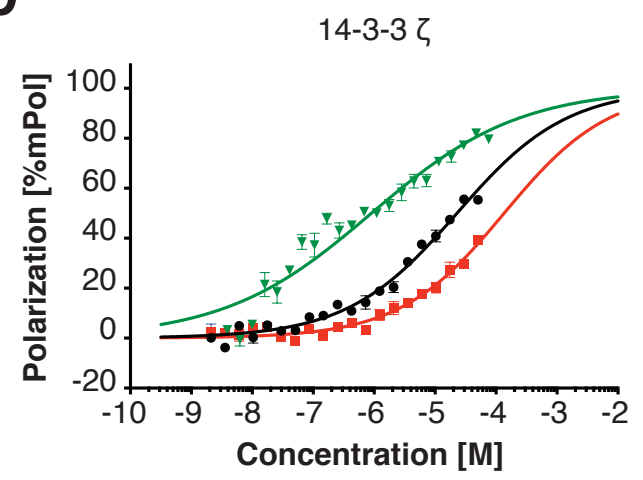

$\mathbf{F}$

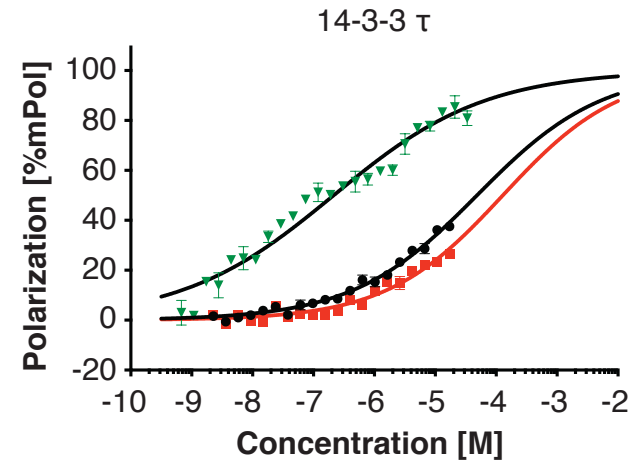

Figure 12: 14-3-3 affinities to TASK-1 WT pS392 are several fold reduced compared to TASK-1 WT pS393. A to $\mathbf{G}$ illustrate equilibrium binding isotherms obtained for respective interaction pairs of TASK-1 WT pS392 peptides (red line), TASK-1 WT pS393 (black line) and TASK-3 WT pS373 (green line) with different 14-3-3 isoforms. 


\section{Results}

The assay was repeated at least six times with two batches of independently purified protein. Error bars depict standard error of the mean (s.e.m.).

Finally, I assessed the functional consequence of double phosphorylation on 14-3-3 binding, employing TASK-1 WT pS392pS393 peptides. I utilized the same experimental approach and compared binding parameters obtained for differentially phosphorylated TASK-1 derived peptides (Figure 13). Calculated affinities for TASK-1 WT pS392pS393 varied from $92.2 \pm 2.9 \mu \mathrm{M}$ (14-3-3 Figure 13, A; Table 11) to $861 \pm 23 \mu \mathrm{M}$ (14-3-3ع, Figure 13, C; Table 11).

FP: $\mathrm{K}_{\mathrm{D}}[\mu \mathrm{M}]$

TASK-1 WT pS392pS393 $\quad$ TASK-1 WT pS392 $\quad$ TASK-1 WT pS393

\begin{tabular}{|c|c|c|c|c|c|c|c|c|}
\hline $14-3-3 \beta$ & 92.2 & \pm & 2.9 & 33.1 & \pm & 0.7 & 7.5 & \pm \\
\hline $14-3-3 y$ & 190.4 & \pm & 19.0 & 51.4 & \pm & 2.7 & 9.8 & \pm \\
\hline $14-3-3 \varepsilon$ & 861.4 & \pm & 23.0 & 373.6 & \pm & 33.2 & 29.0 & \pm \\
\hline $14-3-3 \zeta$ & 264.4 & \pm & 8.9 & 133.0 & \pm & 1.8 & 23.0 & \pm \\
\hline $14-3-3 n$ & 525.4 & \pm & 120.0 & 81.5 & \pm & 7.8 & 9.2 & \pm \\
\hline 14-3-3т & 662.9 & \pm & 86.3 & 123.2 & \pm & 9.0 & 49.4 & \pm \\
\hline $14-3-3 \sigma$ & 106.2 & \pm & 10.4 & 58.8 & \pm & 5.3 & 36.0 & \pm \\
\hline
\end{tabular}

Table 11: Comparison of affinity binding constants determined for respective interaction pairs of TASK-1 WT pS393, TASK-1 WT pS392, TASK-1 WT pS392pS393 and different mammalian 14-3-3 isoforms. Values are displayed as the average of six experiments. Error depicts standard error of the mean (s.e.m.). 


\section{Results}

A

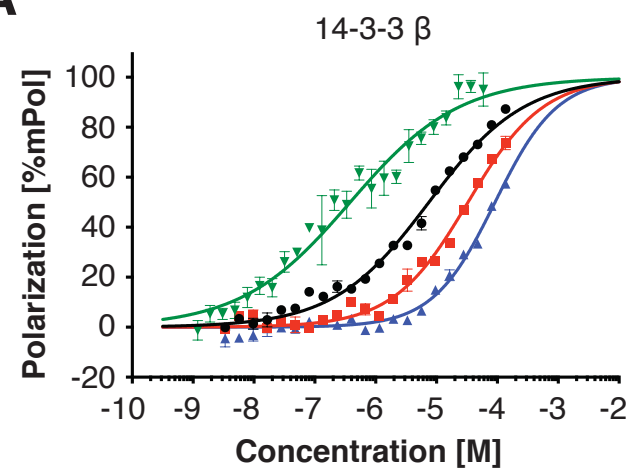

$\mathrm{c}$

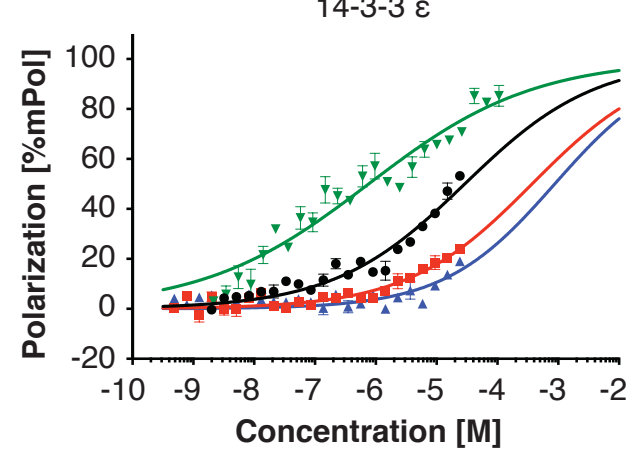

$\mathbf{E}$

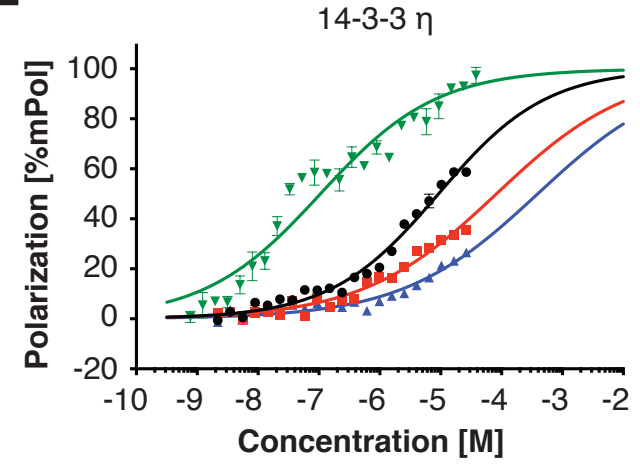

G

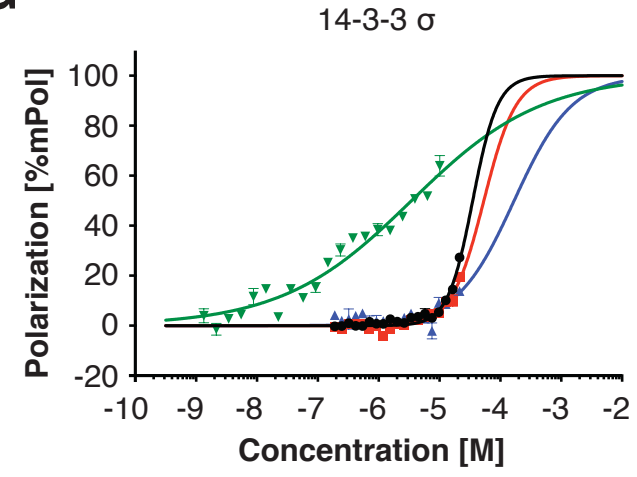

B

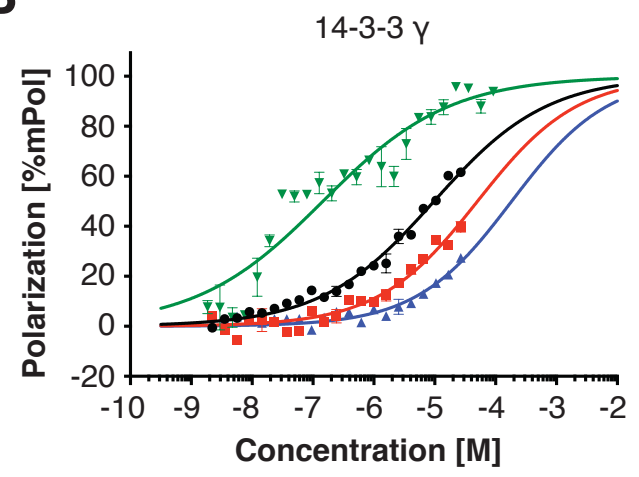

D

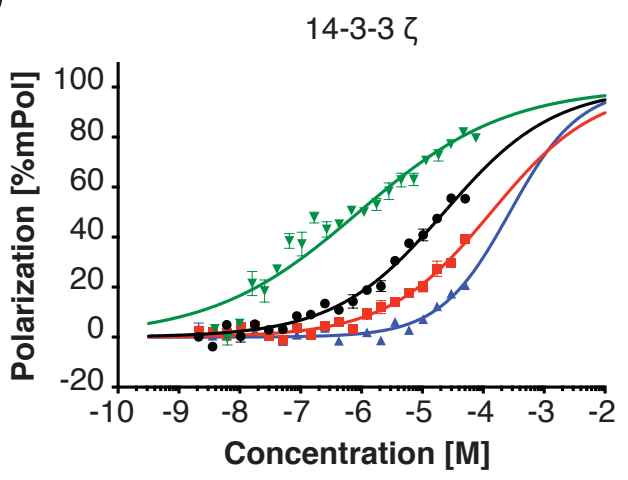

$\mathbf{F}$

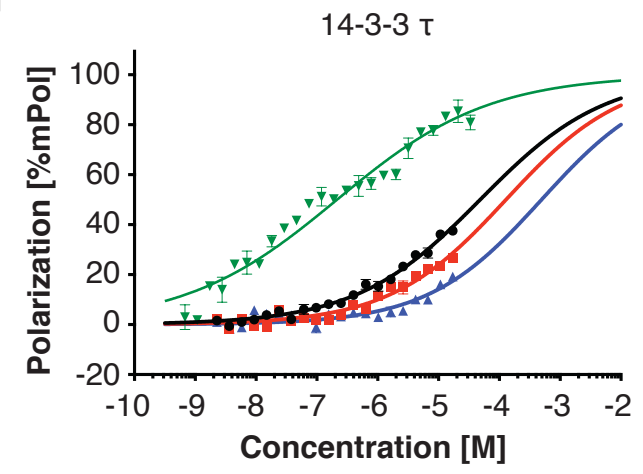

$\rightarrow$ TASK3 pS373

- TASK1 pS393

- TASK1 pS392

- TASK1 pS392pS393

Figure 13: Double phosphorylation of the TASK1 C-terminus results in strongly reduced binding affinities of 14-3-3 proteins indicating a potential inhibitory function of S392 on 14-3-3 binding. A to $\mathbf{G}$ illustrate equilibrium binding isotherms obtained for respective interactions pairs of TASK-1 WT pS392pS393 (blue line), TASK-1 WT 
pS392 peptides (red line), TASK-1 WT pS393 (black line) and TASK-3 WT pS373 (green line) with different 14-3-3 isoforms. The assay was repeated at least six times with two batches of independently purified protein. Error bars depict standard error of the mean (s.e.m.).

A comparison of 14-3-3 binding affinities for TASK-1 WT pS392pS393, TASK1 WT pS392 and TASK-1 WT pS393 clearly shows the impact of phosphorylation of S392 on 14-3-3 binding. Compared to TASK-1 WT pS392

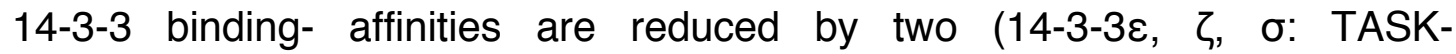
1 WT pS392pS393 - TASK-1 WT pS392) to six fold (14-3-3n: TASK-1 WT pS392pS393 - TASK-1 WT pS393). More obvious differences are observed comparing 14-3-3 affinities determined for TASK-1 WT pS392pS393 and TASK-1 WT pS393. Between individual 14-3-3 isoforms, differences in affinity, ranging from three (14-3-3o: TASK-1 WT pS392pS393 - TASK-1 WT pS393) to 60-fold (14-3-3n: TASK-1 WT pS392pS393 - TASK-1 WT pS393), were observed.

Taken together I show that the presence of a second Serine residue (S392) preceding the conserved Serine of the mode III 14-3-3 binding motif (S393) in TASK-1 reduces binding affinities by several orders of magnitude compared to binding affinities determined for TASK-3. I further conclude that in vitro, upon phosphorylation of S392, 14-3-3 binding to TASK-1 derived C-terminal peptides is inhibited, indicating a potential regulatory role of this residue (S392) in vivo. 


\section{Results}

\section{Correlation of 14-3-3 binding parameters by Surface Plasmon}

Resonance (SPR)

To confirm my findings by another quantitative method, I utilized surface plasmon resonance (SPR). The last 15 amino acids of the distal TASK-3 Cterminus were $\mathrm{N}$-terminally fused to a glutathione S-transferase (GST) proteintag, recombinantly expressed and affinity purified from Escherichia coli (E.coli). Since the proteins were expressed in bacteria they were not phosphorylated. To remove contaminants, affinity purified proteins were subjected to size exclusion chromatography. To study phosphorylation dependent binding of 14-3-3 proteins, we developed an on-chip phosphorylation assay based on studies by Mant et al., 2011, reporting that TASK-1 and TASK-3 C-terminal peptides are efficiently phosphorylated by the catalytic subunit of PKA, in vitro (23).

I assessed 14-3-3 binding of all seven mammalian 14-3-3 isoforms to GSTTASK-3 WT before and after phosphorylation. Figure 14 illustrates a basic experimental setup, used to study phosphorylation dependent 14-3-3 binding to GST-TASK-3 WT fusion proteins.

For this assay, a polyclonal anti-GST antibody (Carl Roth, Karlsruhe, Germany, 3998.1) was crosslinked to the SPR sensorchip surface at high density (109). The sensorchip surface was washed with glycine to remove contaminants and equilibrated in SPR running buffer. TASK-3 WT GST-fusion protein (the ligand) was injected over the surface at a flow rate of $30 \mu \mathrm{l} / \mathrm{min}$ and captured onto the chip surface at low density. Affinity captured ligand proteins were equilibrated in SPR running buffer and subsequently phosphorylated by injection of a phosphorylation solution containing an ATP regeneration system (108) and recombinant catalytic subunit of PKA. Following phosphorylation, contaminants were rinsed from the surface by multiple buffer injections. 
A

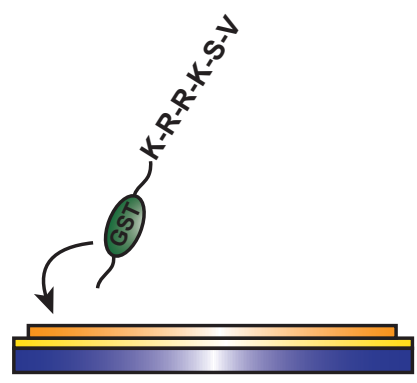

1. Immobilization

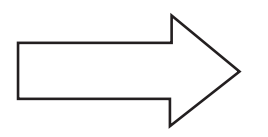

B

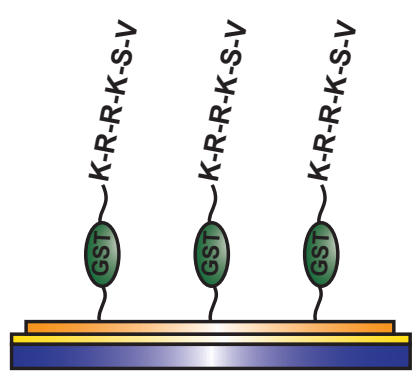

Binding experiment 1

C

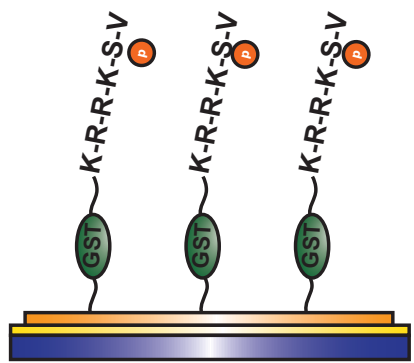

Binding experiment 2

Figure 14: Experimental setup of a Surface Plasmon Resonance (SPR) assay used to study phosphorylation dependent 14-3-3 binding to TASK-3 WT C-termini. A: Capture of the ligand onto the chip surface, which, prior to ligand capture, was modified with a polyclonal anti-GST antibody, at low density. B: Binding experiments performed after capture. At this stage 14-3-3 proteins did not bind to the unphosphorylated TASK3 C-terminus leading to the conclusion, that 14-3-3 proteins binding occurs exclusively in a phosphorylation dependent manner. C: Following capture, the ligand was phosphorylated by injection of a phosphorylation mix containing PKA and ATP followed by incubation for 20 minutes at $20^{\circ} \mathrm{C}$. 14-3-3 proteins were titrated and injected over the surface. At this stage binding of 14-3-3 proteins could be observed following the change of recorded response. Representative sensograms for various interactions measured are shown in Figure 15 and Figure 17. 


\section{Results}

Effective phosphorylation of the ligand was assessed employing a polyclonal phospho-PKA substrate antibody, raised against phosphorylated PKA consensus sites. The antibody was serially diluted and injected over the surface. High affinity binding of this antibody was observed with a dissociation constant of $K_{D}=4.5 \pm 0.6 \mathrm{nM}$ (Figure 15)

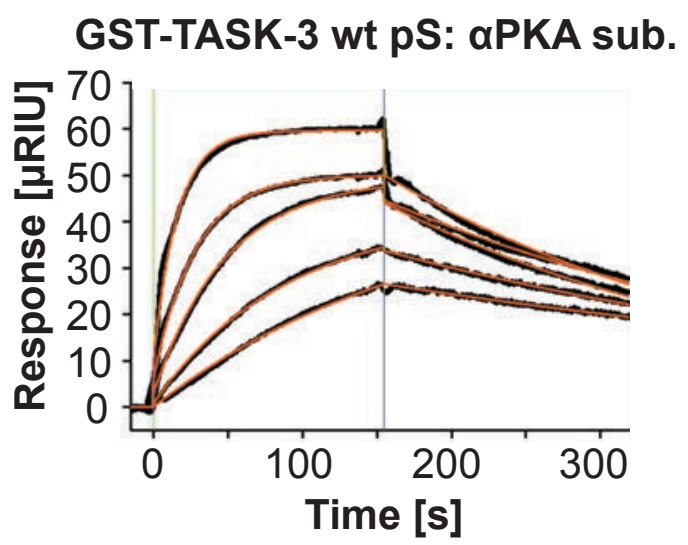

Figure 15: The surface captured GST-TASK3 WT fusion protein is efficiently phosphorylated by recombinantly expressed catalytic subunit of PKA. GST-TASK3 WT fusion protein was captured onto the surface of a modified SPR biosensor chip, at low density. Subsequently, after capture and phosphorylation of the ligand (GSTTASK3 WT) by injecting PKA, a polyclonal anti-GST Antibody was titrated in running buffer and injected over the surface. The analyte was injected at concentrations of: $100 \mathrm{nM}, 50 \mathrm{nM}, 25 \mathrm{nM}, 12.5 \mathrm{nM}$ and $6.25 \mathrm{nM}$. The response was recorded (black line) and analyzed applying a simple 1:1 Langmuir interaction model (orange line). The affinity binding constant was determined with $\mathrm{K}_{\mathrm{D}}=4.5 \pm 0.6 \mathrm{nM}$. The surface was regenerated with Glycine $\mathrm{pH} 2.2$ after each analyte injection (anti-GST antibody). The assay was repeated three times. The error is depicted as standard error of the mean (s.e.m.).

I next evaluated 14-3-3 binding before and after treatment with PKA, which allowed me to indirectly confirm that the ligand was phosphorylated. No 14-33 binding to the unphosphorylated C-terminus of TASK3 was observed, whereas upon phosphorylation, using recombinantly expressed catalytic subunit of PKA, all 14-3-3 isoforms bound the ligand (GST-TASK-3 WT) with 
high affinity. Determined dissociation constants varied from $45 \pm 9 \mathrm{nM}$ (14-3$3 \gamma)$ to $742 \pm 29 \mathrm{nM}(14-3-3 \sigma)$.

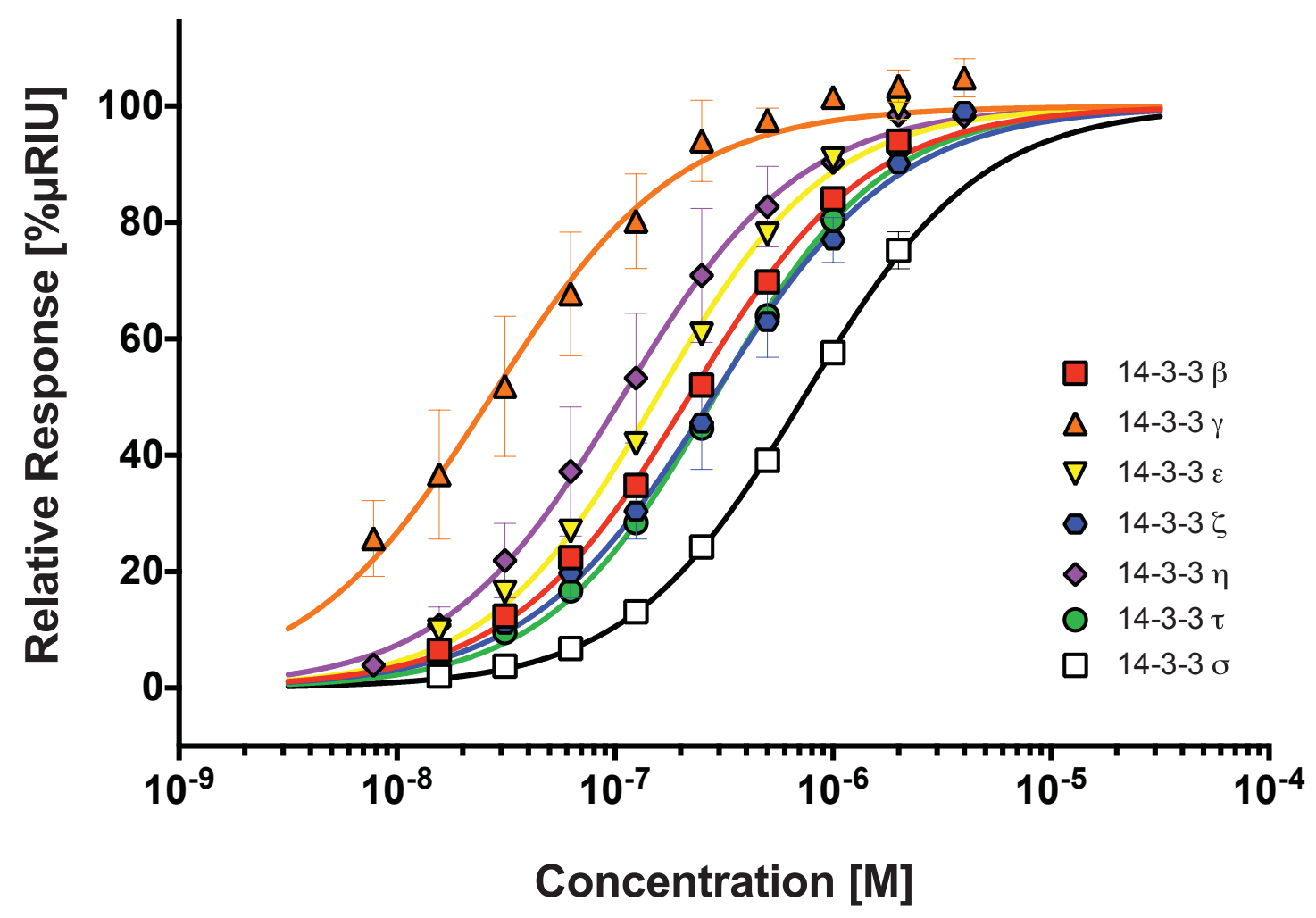

Figure 16: Equilibrium binding isotherms (Dose-response curves) obtained for respective interaction pairs of GST-TASK3 WT with individual 14-3-3 protein isoforms by SPR. The data was analyzed applying a sigmoidal dose-response curve fit (monophasic, Goodness-of-fit: $\mathrm{R}^{2}$ from 0.96 to 0.99 ). Calculated affinity-binding constants are listed in Table 12. The assay was repeated six times for each data point with two batches of independently purified protein. Error bars depict standard error of the mean (s.e.m.).

In general, differences between isoforms in binding affinity observed by fluorescence polarization (FP) were maintained comparing affinities determined by both methods. 14-3-3y and 14-3-3n bound the TASK-3 WT Cterminus with higher affinity than the other isoforms, while 14-3-3 $\sigma$ bound with a significantly lower affinity then all other isoforms tested. Absolute values 


\section{Results}

obtained by FP and SPR varied between two-fold (14-3-3 $\beta$ ) and five-fold (14$3-3 \varepsilon, 14-3-3 \zeta$ and 14-3-3o, Table 12).

These differences should not to be overrated, since small differences can be explained by a different steric presentation of the C-terminal peptide, immobilized at a solid interface (SPR) and in solution (FP). We conclude, that both methods yield comparable binding parameters, maintaining differences between isoforms and demonstrating high affinity binding of all 14-3-3 isoforms to the TASK-3 WT C-terminus. Corresponding equilibrium binding isotherms are depicted in Figure 16. A direct comparison of equilibrium binding constants determined by SPR and FP is illustrated in Table 12 . In Figure 17 representative sensograms obtained for respective interaction pairs of GST-TASK-3 WT and different 14-3-3 isoforms are displayed.

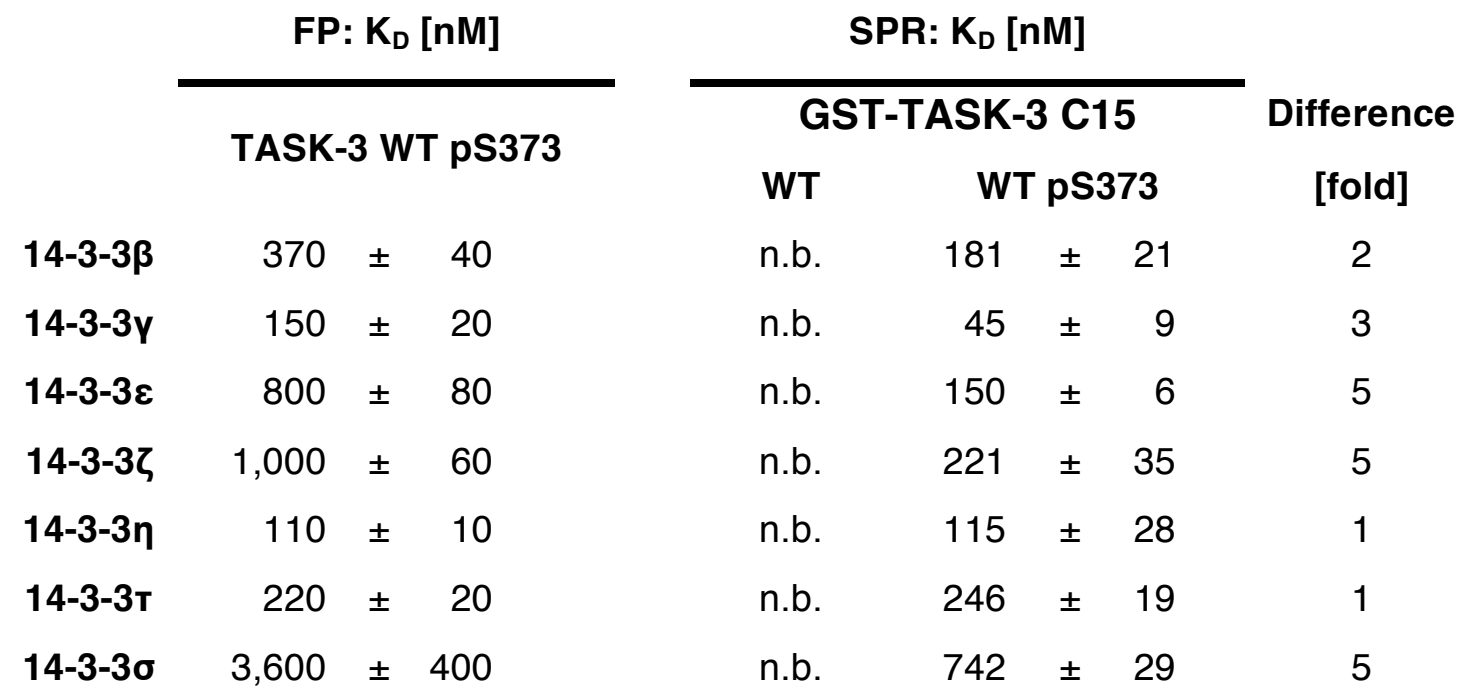

Table 12: Affinity binding constants determined by Fluorescence polarization titration and Surface Plasmon Resonance. Values display the average of six measurements, error depicts standard error of the mean (s.e.m.). 
A

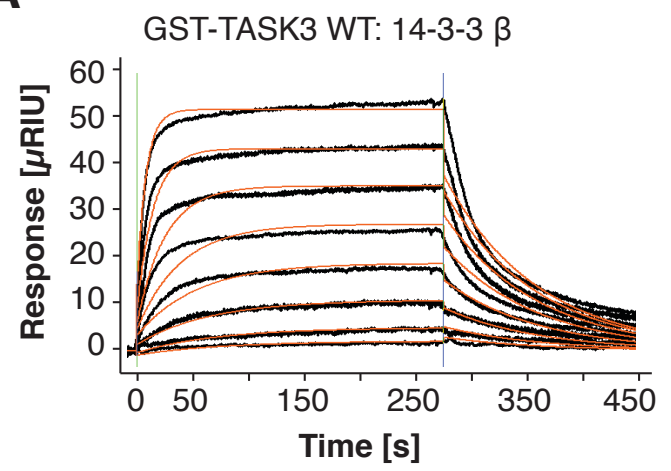

C

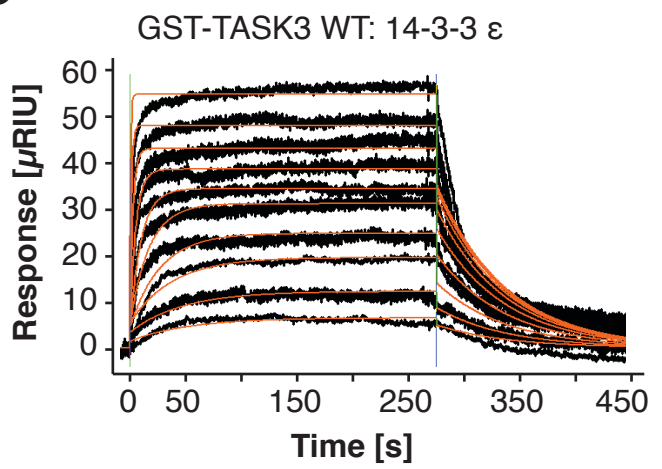

$\mathbf{E}$

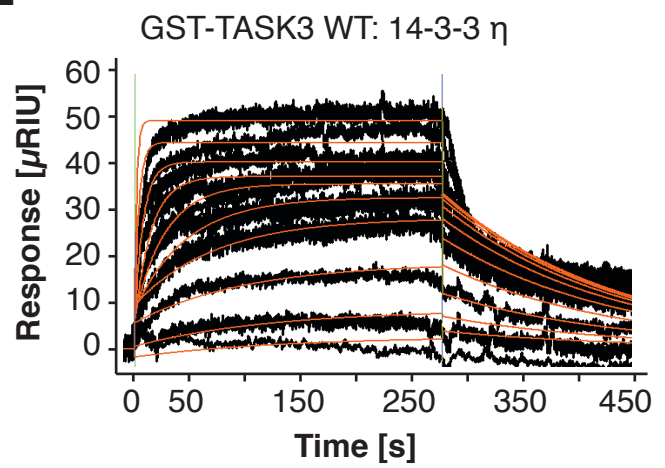

G

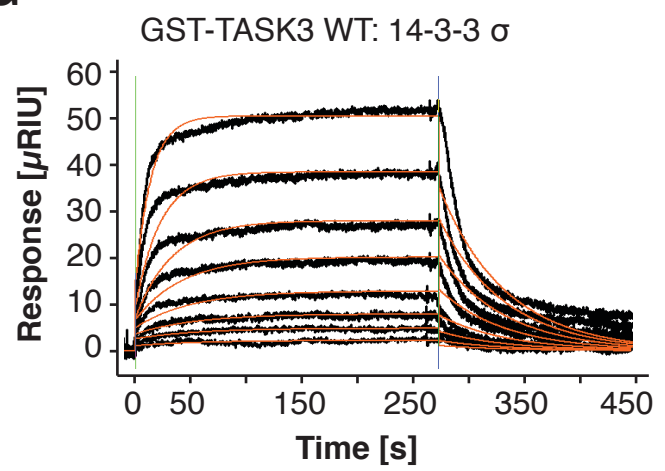

B

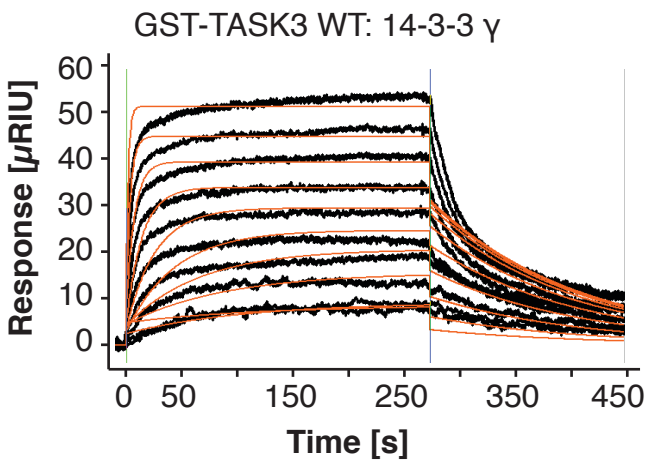

D

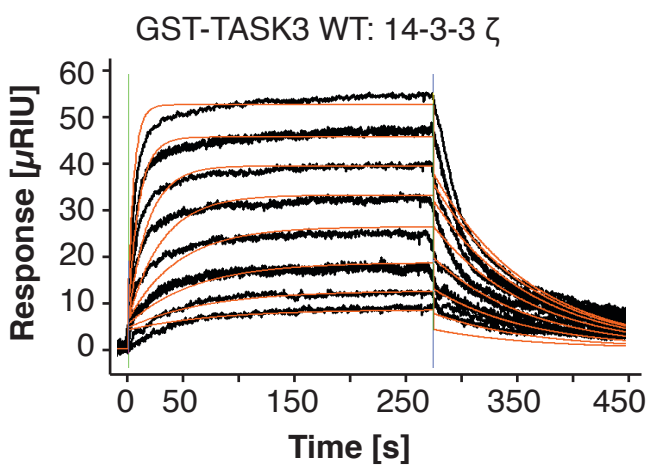

$\mathbf{F}$

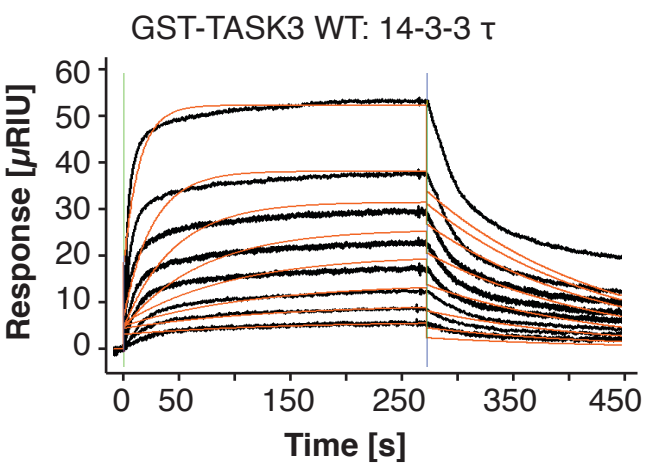

Figure 17: Binding parameters obtained by fluorescence polarization (FP) were corroborated using Surface Plasmon Resonance (SPR). 14-3-3 proteins bind to the phosphorylated and immobilized TASK3 C-terminus with high affinity, maintaining differences between isoforms, previously observed by FP. Representative 


\section{Results}

sensograms obtained by SPR for respective interaction pairs of GST-TASK-3 WT with individual 14-3-3 isoforms are displayed in $\mathbf{A}$ to $\mathbf{G}$. The analyte was injected for 4:30 minutes and the dissociation of the protein from the surface was tracked for 7:00 minutes (displayed dissociation time $\sim 4$ minutes). 14-3-3 concentrations were titrated in a range from $15 \mathrm{nM}$ to $4 \mu \mathrm{M}$. The obtained response data (black line) was analyzed applying a simple 1:1 Langmuir interaction model (orange line). Kinetic parameters determined are listed in Table 12. The assay was repeated at least six times with two batches of independently purified protein. Error values are depicted as standard error of the mean (s.e.m.).

\section{TASK-1 and TASK-3 C-termini are phosphorylated by PKA in vitro}

After detailed characterization of phosphorylation-dependent 14-3-3 binding to various TASK-1 and TASK-3 derived clients quantitatively, we were further interested in understanding upstream regulatory events, mediating the phosphorylation of 14-3-3 client proteins in vivo. Our particular interest was focused on PKA catalyzed phosphorylation of different TASK-derived client proteins. It is well established that the catalytic subunit of PKA (PKAc, further referred to as PKA) phosphorylates TASK-1 and TASK-3 C-terminal peptides in vitro (23).

First I investigated phosphorylation of TASK-1 derived GST-fusion proteins in vitro. I further confirmed that PKA efficiently phosphorylates S392 and S392 in vitro, employing GST-TASK-1 CT15 fusion proteins, with either one (GSTTASK-1 S392A, GST-TASK-1 S393A), or both Serine residues (GST-TASK-1 WT) accessible to phosphorylation by PKA (Figure 18).

GST-TASK fusion proteins (TASK-1 WT, TASK-1 S392A and TASK-1 S393A) were used to conduct in vitro phosphorylation assays employing PKA. Phosphorylation experiments were performed with PKA in presence and absence of ATP. Samples incubated with PKA excluding ATP from the phosphorylation reaction were used as a control, in which the protein was 
essentially not phosphorylated. Figure 11 shows the GST-fusion proteins after PKA treatment and separation on a standard SDS-PAGE (lower panel) or Phostag SDS-PAGE gel (upper panel). For GST-TASK-1 WT two distinct protein bands were observed. The first band migrated at a position corresponding to the GST-fusion protein treated with PKA in absence of ATP. The second protein band observed was shifted upward relative to control incubations performed in absence of ATP (migrating at $\sim 42 \mathrm{kDa}$ in size), indicating that the TASK-1 fusion protein was efficiently phosphorylated by PKA. Similar observations were made for GST-TASK-1 S392A and GSTTASK-1 S393A. For each fusion protein two protein bands were observed after separation on a Phostag SDS-PAGE gel. The first band migrated at the same height as control incubations made in absence of ATP and the the second band observed was shifted upward relative to the first band indicating retardation of the protein on Phostag SDS-PAGE gels due to efficient phosphorylation of the protein. The phosphorylation band observed for either GST-TASK-1 S392A, or GST-TASK-1 S393A migrated faster than the corresponding phosphorylation band observed for GST-TASK-1 WT, indicating a different phosphorylation state of the GST-fusion protein. Differences observed indicate that the TASK-1 WT fusion protein is double phosphorylated, whereas the migratory behavior of each mutant indicates a single phosphorylation of the protein.

These findings illustrate that phosphorylation of TASK-1 derived GST-fusion proteins is dependent on the presence of PKA and ATP. Furthermore these results demonstrate, that S392 and S393 are efficiently phosphorylated by PKA in vitro, confirming findings reported by Mant et al. 2011 (23). Comparing the migration of GST-TASK-1 WT, GST-TASK-1 S392A and GST-TASK-1 S393A I was also able to distinguish between doubly (GST-TASK-1 WT) and singly phosphorylated proteins (GST-TASK-1 S392A, GST-TASK-1 S393A) after samples were resolved on a Phostag SDS-PAGE gel. Interestingly small differences in migration between GST-TASK-1 S392A and GST-TASK-1 


\section{Results}

S393A were observed, which might be attributed to differences in sterical presentation of the phosphorylated TASK-C-terminus.

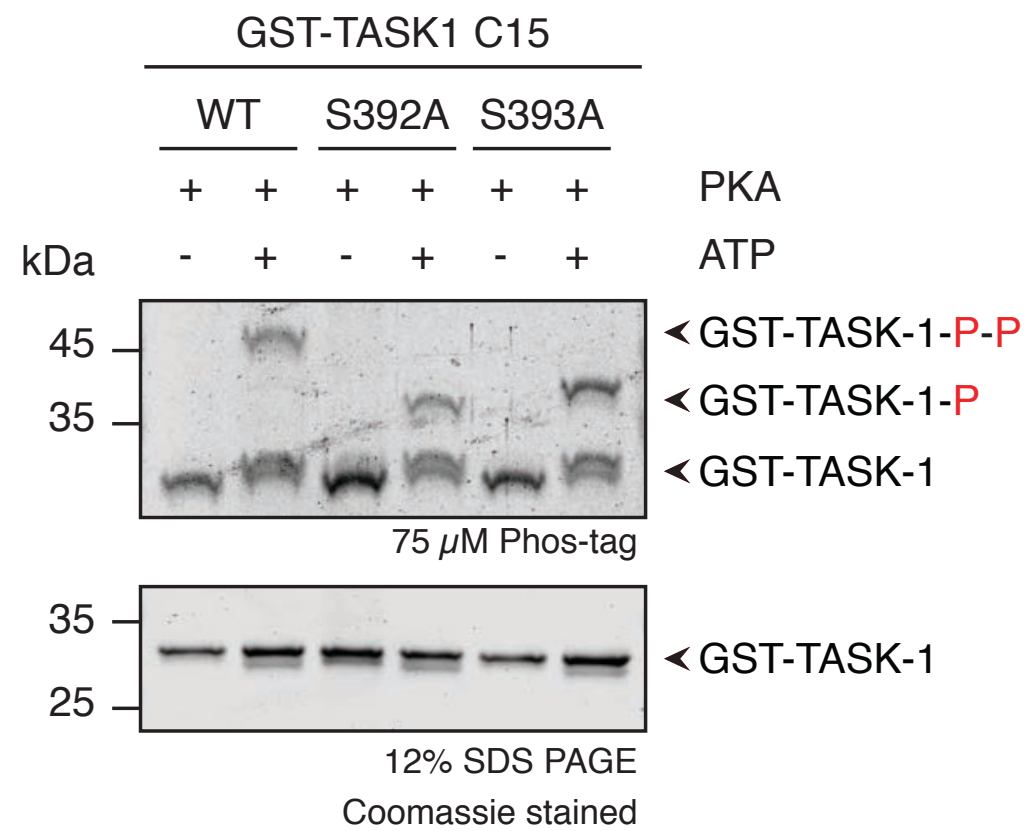

Figure 18: S392 and S393 of TASK-1 are efficiently phosphorylated by the catalytic subunit of PKA (PKAc) in vitro. Affinity purified GST-TASK1 C-terminus fusion proteins (GST-TASK1 WT, GST-TASK-1 S392A and GST-TASK-1 S393A) were phosphorylated with recombinant catalytic subunit of PKA in presence (Lane 2, 4 and 6) and absence of ATP (Lane 1, 3 and 5) for 12 hours at $4^{\circ} \mathrm{C}$, in vitro. Samples were resolved on Phostag SDS PAGE gels, upper panel or standard SDS-PAGE gels, lower panel and subsequently stained with coomassie solution to visualize protein bands. The assay was repeated 6 times. ' $P$ ' suffixes denote the number of phosphorylated residues.

\section{Deletion of the distal Valine V374 in TASK-3 affects PKA} phosphorylation

To evaluate how efficiently TASK-derived proteins are phosphorylated by PKA and how different substrates compare, we generated different TASK-3-derived GST-fusion proteins. We were particularly interested in how phosphorylation of the truncated TASK-3 C-terminus compares to phosphorylation of the wild 
type TASK-3 C-terminus. For this purpose the last 15 amino acids of TASK-3, a truncated version of the TASK-3 C-terminus and a 14-3-3 binding deficient version were fused to the Glutathione-S-transferase (GST) protein-tag, expressed in bacteria and subsequently affinity purified. Since they were expressed in bacteria these proteins were not phosphorylated.

In vitro phosphorylation assays were conducted, as described for TASK-1 derived GST-fusion proteins. First, we investigated phosphorylation of the GST-TASK-3 WT fusion protein and a truncated variant, GST-TASK-3 $\Delta$ V374, with $12 \mathrm{~h}$ of PKA treatment (Figure 19). Similar to observations made for TASK-1 derived GST-fusion proteins, two distinct protein bands for GSTTASK-3 WT as well as GST-TASK-3 $\triangle$ V374 were observed after separation on a Phostag SDS-PAGE gel (Figure 19, Lane 2 and Lane 4, upper panel). The first band, faintly stained by Coomassie solution, migrated at the same height as control incubations performed with PKA in absence of ATP, indicating that a small portion of the protein was not phosphorylated. The second band observed was strongly stained by Coomassie solution and shifted upwards, indicating efficient phosphorylation of the client protein by the catalytic subunit of PKA. After $12 \mathrm{~h}$ of PKA-treatment in presence of ATP, we did not observe significant differences in the relative amounts of protein phosphorylated, neither for GST-TASK-3 WT, nor for GST-TASK-3 $\triangle$ V374. Incubation of GST-TASK-3 S373A fusion protein with PKA in absence, or presence of ATP did not alter the migration of the protein on a Phostag SDSPAGE gel, confirming that the TASK-3 C-terminus is specifically phosphorylated at $\mathrm{S} 373$ by the catalytic subunit of PKA.

After $12 \mathrm{~h}$ of incubation with PKA and ATP, both substrates employed were fully phosphorylated, which is not an unexpected result, since the limiting factor, ATP, was constantly resupplied utilizing an ATP regeneration system (108), allowing PKA to continuously phosphorylate available substrates. In addition, for each substrate faintly stained protein bands corresponding to a 


\section{Results}

protein population, which appeared to be 'phosphorylation resistant', were observed.

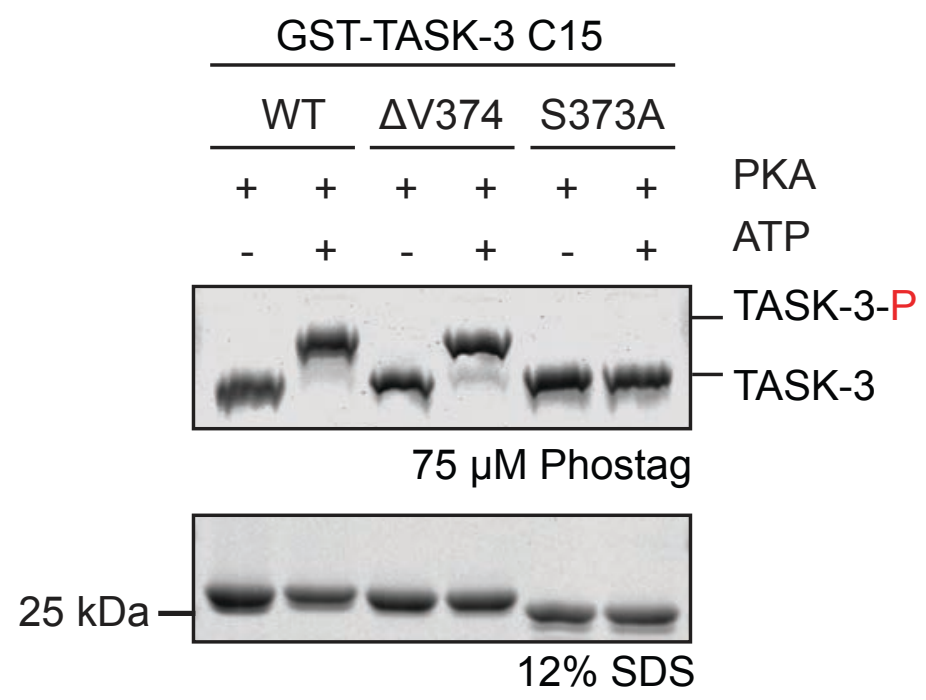

Figure 19: Phosphorylation of TASK-3 derived GST-fusion proteins. After 12 hours of phosphorylation GST-TASK-3 WT and GST-TASK-3 $\Delta$ V374 were phosphorylated efficiently by recombinant PKA. Affinity purified GST-TASK3 WT, $\triangle$ V374 and S373A fusion proteins were phosphorylated for $12 \mathrm{~h}$ at $4^{\circ} \mathrm{C}$, utilizing the recombinant catalytic subunit of PKA. Experiments were carried out in presence of PKA and ATP (Lane 2, 4 and 6), or with PKA in absence of ATP (Lane 1, 3 and 5). Samples were resolved on Phostag SDS PAGE gels, upper panel, or standard SDS-PAGE gels, lower panel and subsequently stained with coomassie solution to visualize protein bands. The assay was repeated three times. ' $P$ ' denotes the number of phosphorylated residues.

To further clarify if both substrates are equally well phosphorylated within a shorter period of time, we quantified the relative amount of protein phosphorylated after 10 minutes of PKA treatment. In vitro phosphorylation assays were conducted as described for GST-TASK-1 fusion proteins and samples were resolved on either Phostag SDS-PAGE or standard SDS-PAGE gels. As described previously, proteins incubated with PKA in absence of ATP were essentially not phosphorylated and migrated faster on Phostag SDS- 
PAGE gels. Whereas the migration of phosphorylated proteins was retarded resolving samples on Phostag SDS-PAGE gels, causing a band shift upwards relative to control incubations performed in absence of ATP.

For GST-TASK-3 WT, two protein bands were observed after separation of the sample on a Phostag SDS-PAGE gel. The first band was faintly stained by Coomassie solution and migrated at the same height as control incubations performed in absence of ATP, indicating that a small portion of the protein was not phosphorylated (Figure 20, Lane 1). The second band observed was strongly stained by Coomassie solution and shifted upwards, relative to control incubations performed with PKA in absence of ATP, indicating that the TASK-3 WT C-terminus was efficiently phosphorylated by the catalytic subunit of PKA within 10 minutes of treatment (Figure 20, Lane 2). Similar to these observations, two protein bands were observed for GST-TASK-3 $\Delta$ V374 after incubation with PKA in presence of ATP. However the first band, corresponding to unphosphorylated protein and migrating at the same height as control incubations performed in absence of ATP, was stained more strongly compared to the analogous protein band observed for GST-TASK-3 WT (Figure 20, Lane 3). The phosphorylated band, migrating at the same height as observed for GST-TASK-3 WT, was faintly stained by Coomassie solution (Figure 20, Lane 4), indicating that the truncated TASK-3 C-terminus was less efficiently phosphorylated than the wild type TASK-3 C-terminus. We quantified the amount of protein phosphorylated by forming the ratio of the amount of protein phosphorylated (upper band) with the total amount of protein loaded (SDS loading control, lower panel). For GST-TASK-3 WT we determined that $\sim 60 \%$ of the protein employed was phosphorylated within 10 minutes of PKA treatment, whereas for GST-TASK-3 $\triangle$ V374 only $\sim 20 \%$ of total protein employed was phosphorylated during the same incubation time, indicating that truncation of the TASK-3 C-terminus affected the efficiency of phosphorylation by PKA. 


\section{Results}

Taken together, I conclude that the TASK-3 WT C-terminus was efficiently phosphorylated by the catalytic subunit of PKA in presence of ATP, confirming results reported by Mant et al., 2011 (23). I further demonstrate that the deletion of the distal valine, which affects 14-3-3 binding (see FP) also affected the efficiency of protein phosphorylation by PKA, reducing the amount of phosphorylated protein detected by three-fold. Figure 20, B illustrates our findings.
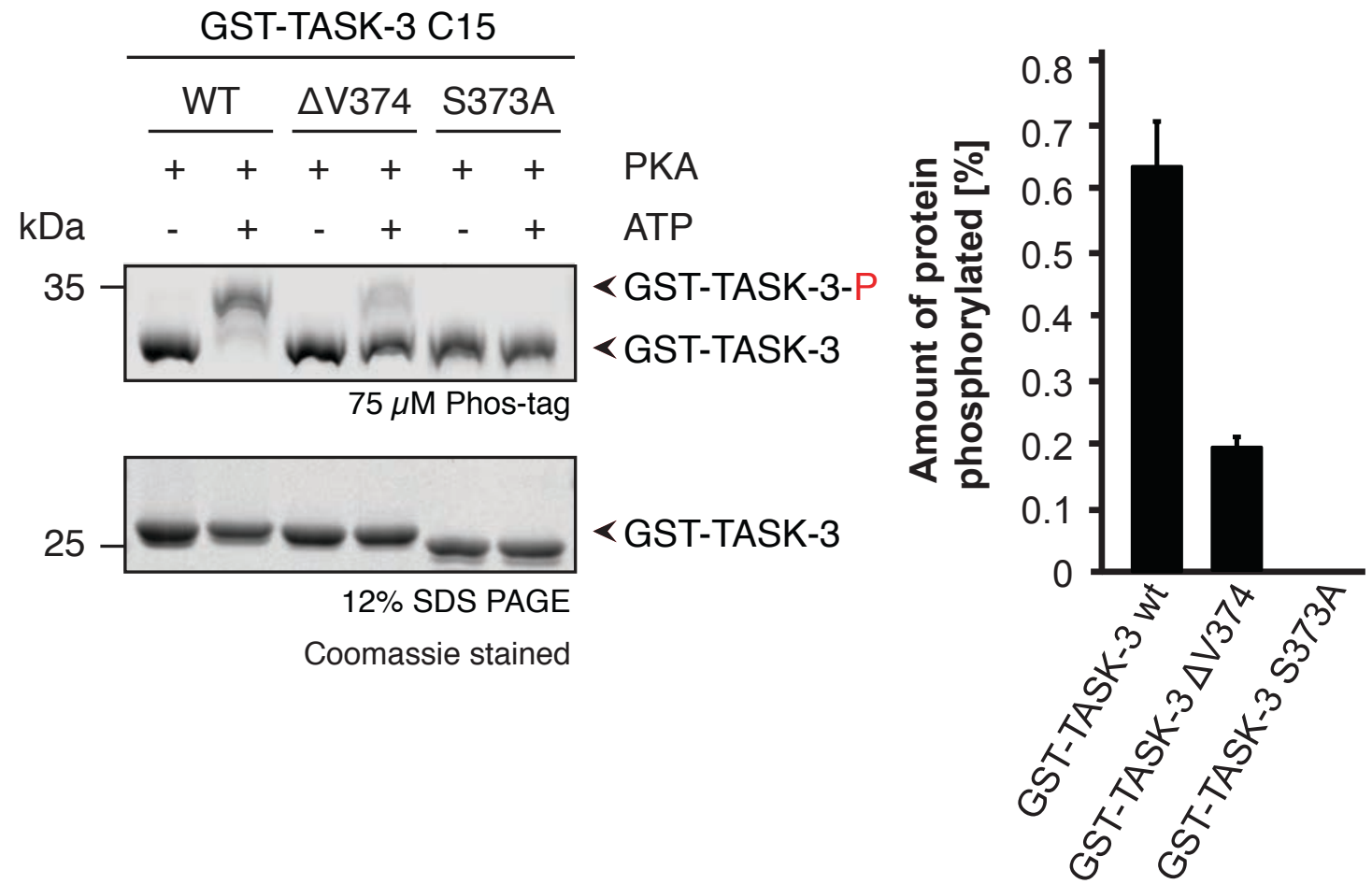

Figure 20: The GST-TASK-3 WT fusion-protein is more efficiently phosphorylated by PKA, than the truncated TASK-3 C-terminus ( $\triangle$ V374). Truncation of the TASK-3 Cterminus reduces the efficiency of phosphorylation by PKA significantly. Affinity purified GST-TASK-3 WT, GST-TASK-3 $\Delta$ V374 and GST-TASK-3 S373A fusion proteins were phosphorylated for 10 minutes at room temperature, utilizing the recombinant catalytic subunit of PKA. Experiments were carried out with PKA in presence of ATP (Lane 2, 4 and 6), or in absence of ATP (Lane 1, 3 and 5). Samples were resolved on Phostag SDS-PAGE gels and SDS-PAGE gels as described above. The total pixel intensity was determined for each protein band. The amount of protein phosphorylated was determined by forming the ratio between values obtained for phosphorylated protein with values obtained for the total protein loaded from SDS- 
PAGE gels. The assay was repeated six times. Error bars depict standard error of the mean (SEM).

\section{Cell surface expression of different TASK-derived reporter proteins}

After broad in vitro characterization of different events regulating cell surface expression of TASK-channels in vivo, we evaluated the functional consequence of mutation of TASK-3 and TASK-1 C-termini on cell surface expression by flow cytometry. We determined the relative cell surface expression of various TASK-derived reporter proteins. Reporter proteins were generated, fusing a fluorescent probe (CFP), as a linker, and the previously described TASK-derived C-terminal peptides to the cytosolic tail of the dimeric surface glycoprotein CD8. The fluorescent linker was introduced, to later on identify cells expressing the reporter protein. All constructs used to study cell surface expression of TASK-derived reporter proteins are listed in Table 13.

Construct

CD8-CFP-TASK-3 WT

CD8-CFP-TASK-3 $\triangle \mathrm{V} 374$

CD8-CFP-TASK-3 S373A

CD8-CFP-TASK-3 K369A

CD8-CFP-TASK-1 WT

CD8-CFP-TASK-1 S392A

CD8-CFP-TASK-1 S393A

CD8-CFP-TASK-1 K389A

CD8-CFP-TASK-1 $\Delta$ V374
C-terminal tail attached

SFTDHQRLMKRRKSV-COOH

SFTDHQRLMKRRKS-COOH

SFTDHQRLMKRRKAV-COOH

SFTDHQRLMARRKSV-COOH

SLSTFRGLMKRRSSV-COOH

SLSTFRGLMKRRASV-COOH

SLSTFRGLMKRRSAV-COOH

SLSTFRGLMARRSSV-COOH

SLSTFRGLMKRRSS-COOH
14-3-3 binding

+++
++
-
+++
+
+
-
+ (predicted)
-

Table 13: Reporter proteins used to study differences in cell surface expression and elucidate the role of different residues and sorting motifs in vivo. Observed 14-3-3 binding is denoted with + : for low affinity binding, ++: for moderate affinity binding, +++ : for high affinity binding, -: for no binding. 


\section{Results}

To investigate the functional consequence of high affinity 14-3-3 binding on cell surface expression of TASK-3-derived reporter proteins, I expressed the TASK-3-derived reporter proteins, listed in Table 13, in COS7 cells. The relative cell surface expression of CD8-CFP-TASK-3 WT, CD8-CFP-TASK-3 S373A, a 14-3-3 binding deficient mutant, CD8-CFP-TASK-3 K369A, a mutant deficient for COPI binding and CD8-CFP-TASK-3 $\triangle$ V374, a truncated version of the TASK-3 C-terminus was determined.

First I assessed the relative cell surface expression of CD8-CFP-TASK-3 WT, therefore I detected the extracellular domain of CD8 utilizing a monoclonal anti-CD8 primary antibody and an Alexa-Fluor-647 coupled secondary antibody. In parallel I assessed the relative surface expression of CD8-CFPTASK-3 S373A, CD8-CFP-TASK-3 K369A and CD8-CFP-TASK-3 $\Delta$ V374. Figure 21 illustrates my observations. The relative amount expressed at the cell surface of each reporter protein was normalized to the TASK-3 WT reporter protein, a bar diagram summarizing my findings is show in Figure 22.

The TASK-3 wild type reporter protein was efficiently transported to the cell surface (Figure 21 A, Q2). Upon mutation of the S373, the conserved Serine residue of the mode III 14-3-3 binding motif, the relative cell surface expression of the reporter protein was decreased significantly (Figure 21 B, Q2). The relative cell surface expression of CD8-CFP-TASK-3 S373A was more than five fold lower compared to the wild type reporter protein. These results confirm that surface expression of the TASK-3 wild type reporter protein depends on the phosphorylation state of S373 and subsequent 14-3-3 binding. Furthermore, the relative cell surface expression of CD8-CFP-TASK$3 \mathrm{~K} 369 \mathrm{~A}$, a COPI binding deficient reporter protein, was assessed (Figure 21 C, Q2). Only minor differences between CD8-CFP-TASK-3 K369A and CD8CFP-TASK-3 WT were observed. Hence the CD8-CFP-TASK-3 K369A reporter reaches the cell surface efficiently, but no longer requires phosphorylation and subsequent 14-3-3 binding to escape retention/retrieval by COPI (11), we conclude that similar levels of the CD8-CFP-TASK-3 WT 
reporter protein must be a result of efficient phosphorylation and subsequent high affinity 14-3-3 binding.

A

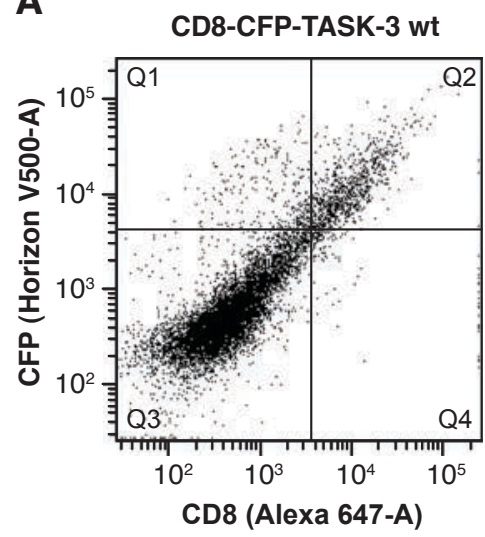

C

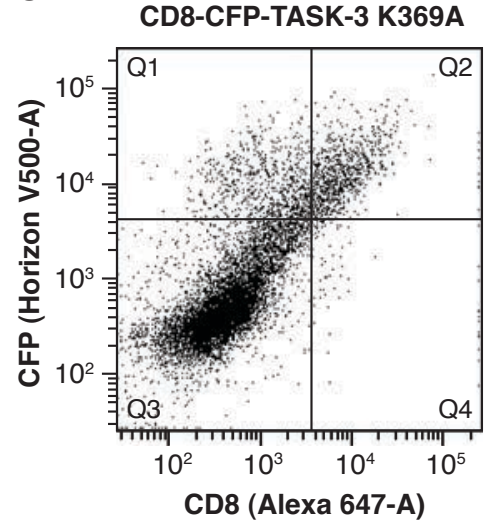

B
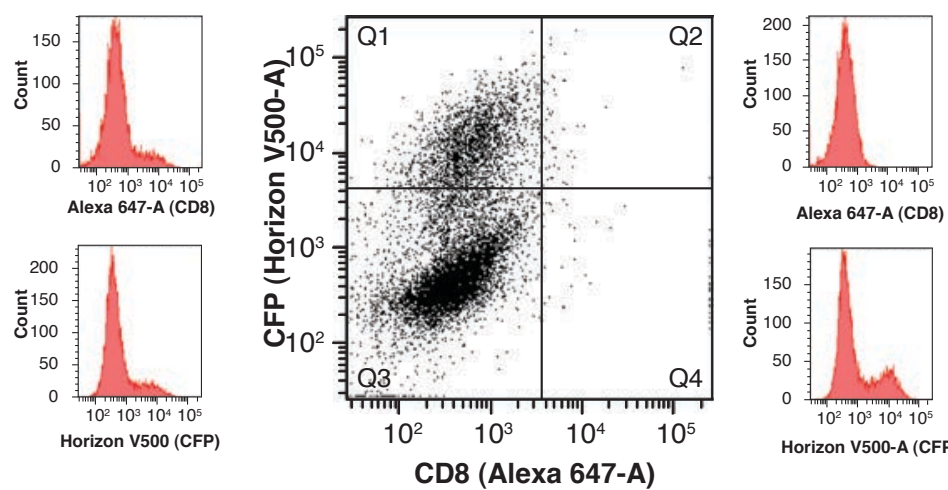

D

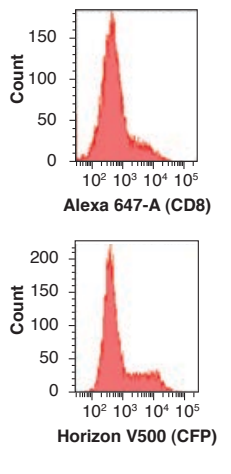

D CD8-CFP-TASK-3 $\Delta$ V374

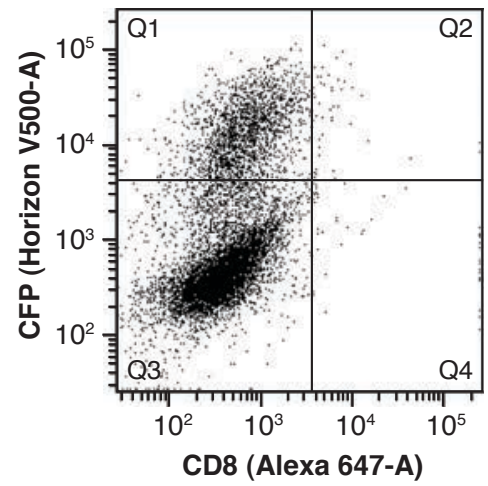

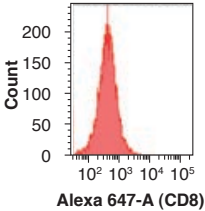

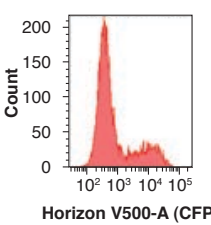

Figure 21: TASK-3 derived reporter proteins are differently expressed at the cell surface. Cos7 cells were transiently transfected with DNA encoding TASK-3 derived reporter proteins. Proteins were expressed for $36 \mathrm{~h}$. Surface localised proteins were detected employing a monoclonal anti-CD8 primary antibody and an Alexa-647 conjugated secondary antibody. Following detection, cells were subjected to Flow cytometry and the relative cell surface expression of different reporter proteins was determined. Displayed are representative dot-plots for each reporter protein employed. Each dot-plot was divided into four quadrants (Q1 - Q4). Q1: CFP-positive cells, cells expressing the reporter, in which the reporter was not detected at the cell surface. Q2: CFP/CD8-positive cells, cells expressing the reporter protein, in these cells the reporter protein was detected at the cell surface. Q3: untransfected cells. Q4: CD8-positive cells, background staining of the CD8 antibody used, these cells are not considered in our analysis. From Q2 a histogram was generated and is shown next to the dot plot (Alexa 647-A), from this histogram the mean of the CD8- 


\section{Results}

signal was calculated. A second histogram was generated for all cells expressing the protein, identified by the CFP emission signal (Horizon V500-A), Q1 and Q2. The relative cell surface expression was expressed as a ratio of the mean of the CD8 signal with the mean of the CFP signal in CFP-positive cells. All values were normalized to the TASK-3 wild-type construct.

I have shown by in vitro binding assays that truncation of the TASK-3 Cterminus does not abolish 14-3-3 binding. To elucidate the functional consequence of truncation on 14-3-3 dependent cell surface expression of the reporter protein, I transiently transfected Cos7 cells with DNA encoding a truncated version of the TASK-3 reporter protein, CD8-CFP-TASK-3 $\Delta$ V374, (Figure 21 D) and determined the relative cell surface expression of the reporter protein after 36 hours of protein expression. In agreement with current literature, the cell surface expression of this reporter protein was significantly reduced compared to the wild-type reporter protein. 


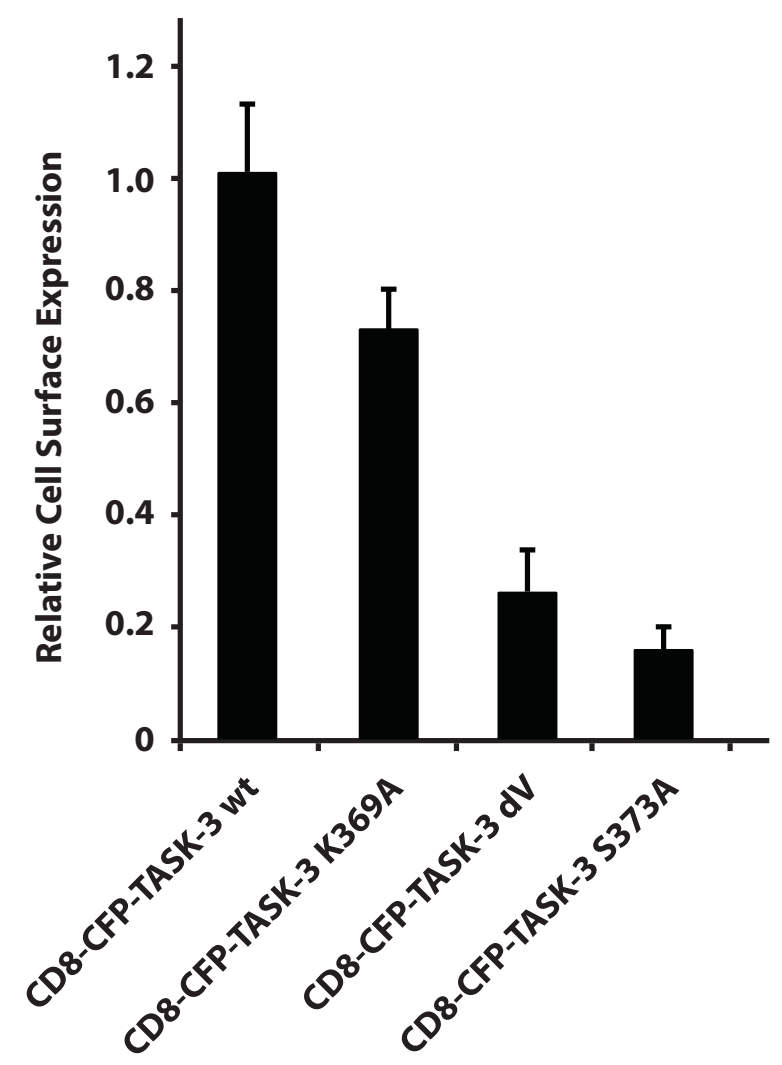

Figure 22: Relative cell surface expression of all TASK3 derived reporter proteins normalized to the TASK3 WT construct. The mean signal intensity of the anti-CD8 signal (Alexa 647) was expressed as a ratio with the mean signal intensity of the CFP signal (Horizon V500) in CFP-positive cells and normalized to the wild type TASK-3 construct. Significant differences in cell surface expression between the TASK3 WT construct and TASK3 $\triangle$ V374, as well as TASK3 S373A were observed. Differences between CD8-CFP-TASK-3 WT and CD8-CFP-TASK-3 K369A are not significant. Bar plots are representative of six independent transfections. Error bars depict standard error of the mean (s.e.m.).

Taken together, minor differences were observed between CD8-CFP-TASK-3 WT (Figure 22, $1^{\text {st }}$ Column) and a COPI-binding deficient mutant (CD8-CFPTASK-3 K369A, Figure 22, $2^{\text {nd }}$ Column), indicative of efficient anterograde trafficking of the wild type reporter-protein along the secretory pathway in a phosphorylation and 14-3-3-dependent manner. The relative cell surface expression of CD8-CFP-TASK-3 S373A and CD8-CFP-TASK-3 $\Delta$ V374 was 


\section{Results}

significantly reduced, compared to levels observed for the wild-type reporterprotein, varying from $\sim 30 \%$ for CD8-CFP-TASK-3 $\triangle$ V374 (Figure 22, $3^{\text {rd }}$ Column) to $\sim 20 \%$ for CD8-CFP-TASK-3 S373A (Figure 22, $4^{\text {th }}$ Column). In general, a reduction of the relative cell surface expression of these reporter proteins by four-fold for CD8-CFP-TASK-3 $\Delta$ V374 and more than five-fold for CD8-CFP-TASK-3 S373A was observed, compared to CD8-CFP-TASK-3 WT. Hence, it appears that the reported lack of cell surface expression observed for TASK-3 reporter constructs lacking the C-terminal valine $(11,17,18)$, can only insufficiently explained by reduced 14-3-3 binding. To fully understand the regulatory mechanism retaining/retrieving the protein, other factors, such as phosphorylation of the protein by kinases, dephosphorylation of the protein by phosphatases in relation to 14-3-3-binding and its role in protecting the client protein from dephosphorylation (1), need to be considered.

\section{Deletion of V374 decreases the efficiency by which PKA phosphorylates the TASK-3 C-terminus in vivo}

We analyzed the phosphorylation state of each TASK-3-derived CD8-reporterprotins, expressing each reporter protein in COS7 cells. Cells were lysed after 36 hours of protein expression, centrifuged and crude membranes were solubilized with $1.5 \%$ Triton $\mathrm{X}-100$ and $0.1 \%$ SDS. Solubilized membranes were precipitated and the obtained membrane protein pellet was resuspended in phosphatase buffer. For each reporter-protein one sample was treated with $\lambda$-protein phosphatase ( $\lambda$ PPase). Samples were subsequently resolved on a Phostag SDS-PAGE gel, as well as a standard SDS-PAGE gel. Proteins were transferred to a membrane and CD8-reporter proteins were detected using a CD8-a-antibody (Santa Cruz Biotechnology, Santa Cruz, CA; clone H-160, catalog number SC-7188, lot number E2213) and a fluorescently labeled secondary antibody. Membranes were developed using an Odyssey LiCOR imaging system (LiCOR, Bad Homburg, Germany) 


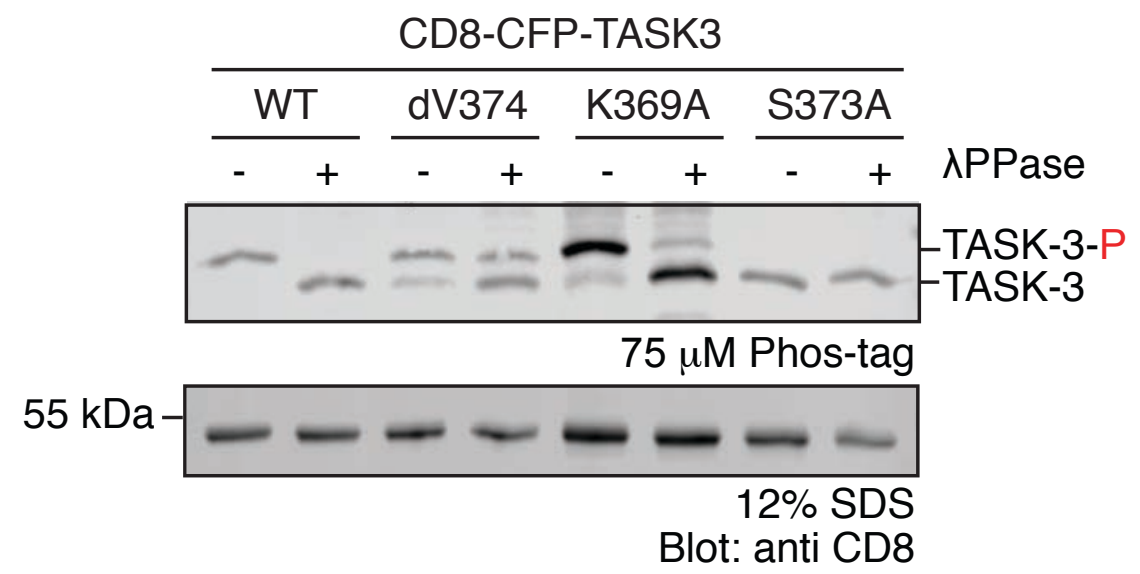

Figure 23: In vivo phosphorylation of indicated TASK-3-derived CD8-reporterproteins. Precipitated proteins were treated with $\lambda$-protein phosphatase ( $\lambda$ PPase) and subsequently separated on either a standard SDS-PAGE, or Phostag SDS-PAGE gel. The phosphorylation state of each reporter protein was assessed by detection of proteins with a CD8-a-antibody and a fluorescently labeled secondary antibody. Phosphorylation bands are indicated by the upward band shift relative to samples treated with $\lambda$ PPase on Phostag SDS-PAGE gels (upper panel). The experiment is representative for 11 independent transfections. TASK-3: unphosphorylated TASK-3derived reporter-protein. TASK-3-P: phosphorylated TASK-3-derived reporter-protein.

For the CD8-CFP-TASK-3 WT reporter-protein one protein band shifted upwards relative to a sample treated with $\lambda$-protein phosphatase ( $\lambda$ PPase) was observed (Figure 23, Lane 1 and Lane 2), confirming that the reporterprotein was efficiently phosphorylated in vivo, in agreement with observations made for in vitro phosphorylation assays performed with GST-TASK-3 WT fusion proteins and recombinantly expressed catalytic subunit of PKA compare to Figure 19 and Figure 20. The COPI binding deficient reporterprotein, CD8-CFP-TASK-3 K369A, was as equally well phosphorylated as CD8-CFP-TASK-3 WT. Treatment with $\lambda$ PPase altered the migratory behavior of the reporter protein (Figure 23, Lane 5, upper band) to match that of CD8CFP-TASK-3 S373A (Figure 23, Lane 6, lower band). The migration of a 143-3-binding deficient reporter-protein, CD8-CFP-TASK-3 S373A, was unaltered in presence or absence of $\lambda$ PPase, after separation on Phostag 


\section{Results}

SDS-PAGE gels. These observations are consistent with observations made for in vitro phosphorylation assays performed with GST-TASK-3 S373A fusion protein and recombinant PKA in presence and absence of ATP. Comparing the phosphorylation state of CD8-CFP-TASK-3 WT and CD8-CFP-TASK$3 \Delta$ V374 substantial differences in the amount of protein phosphorylated were observed. In absence of $\lambda$ PPase only a fraction of the $\Delta V 374$ reporter protein was phosphorylated by cellular kinases (approximately 60\%, Figure 23, Lane $3)$, whereas treatment with $\lambda$ PPase only partially altered the migratory behavior of the reporter protein, indicating inefficient dephosphorylation of the truncated reporter protein (Figure 23, Lane 4). These findings indicate, that a substantial amount of the reporter-protein expressed in Cos7 cells was indeed phosphorylated in vivo. Differences might be explained by considering other factors, such as kinases, phosphatases and interaction partners protecting the client protein from dephosphorylation, dynamically regulating the steady state phosphorylation of reporter proteins such as CD8-CFP-TASK-3 $\Delta$ V374.

\section{Cell surface expression of TASK-1-derived reporter proteins reveals a potential regulatory role of $\mathbf{S} 392$}

I performed additional flow cytometry assays to evaluate the functional consequence of low affinity 14-3-3 binding on cell surface expression of TASK-1-derived reporter proteins. To address the role of S392 and S393 together and individually I employed CD8-CFP-TASK-1 WT (containing both Serine residues), CD8-CFP-TASK-1 S392A and CD8-CFP-TASK-1 S393A reporter proteins, sequentially replacing the corresponding serine residue with alanine. Additionally we determined the relative cell surface expression of a COPI-binding deficient reporter protein, CD8-CFP-TASK-1 K389A and a truncated, 14-3-3 binding deficient reporter protein, CD8-CFP-TASK-1 $\Delta$ V394. In general, experiments were carried out as described for flow cytometry assays conducted with TASK-3-derived reporter-proteins.

The relative cell surface expression of CD8-CFP-TASK-1 WT was substantially lower compared to CD8-CFP-TASK-3 (Figure 24, A, B). These 
observations are in agreement with previous findings reporting differences in cell surface expression of TASK-3 and TASK-1 (11). Replacing S392 with Alanine elevated the relative cell surface expression of the reporter protein to levels comparable to CD8-CP-TASK-3 WT, confirming a potential regulatory/inhibitory role of $\mathrm{S} 392$ on cell surface expression of the reporterprotein (CD8-CFP-TASK-1 S392A, Figure 24, C, Q2). Similar observations were made, replacing $\mathrm{K} 389$ wit Alanine, mutating the ER retrieval/retention motif (CD8-CFP-TASK-1 K389A, Figure 24, D, Q2). Mutation of the conserved Serine residue (S393) of the mode III 14-3-3 binding motif (CD8CFP-TASK-1 S393, Figure 24, E, Q2) on the other hand led to a significant decrease in cell surface expression of the reporter protein compared to CD8CFP-TASK-1, demonstrating that $\mathrm{S} 393$ is required for efficient transport of the protein to the cell surface, consistent with observations made for CD8-CFPTASK-3 S373A. The relative cell surface expression of the truncated reporterprotein CD8-CFP-TASK-1 $\triangle \mathrm{V} 394$ was even further reduced (Figure 24, F, Q2). 


\section{Results}

A

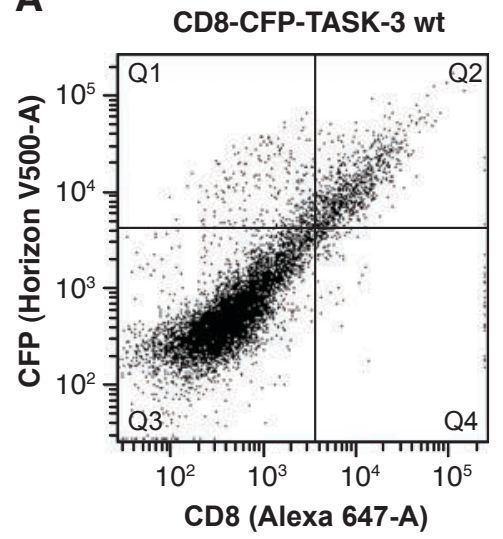

C

CD8-CFP-TASK-1 S392A

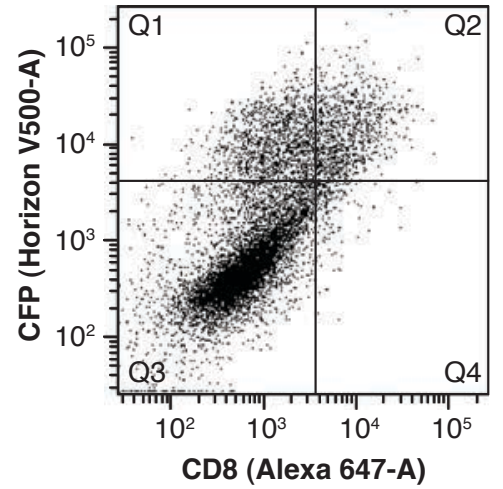

E

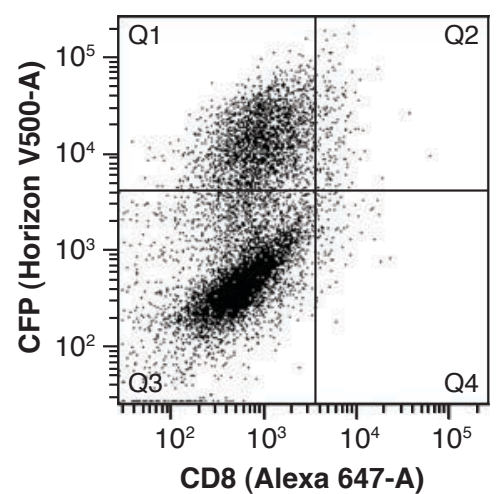

B
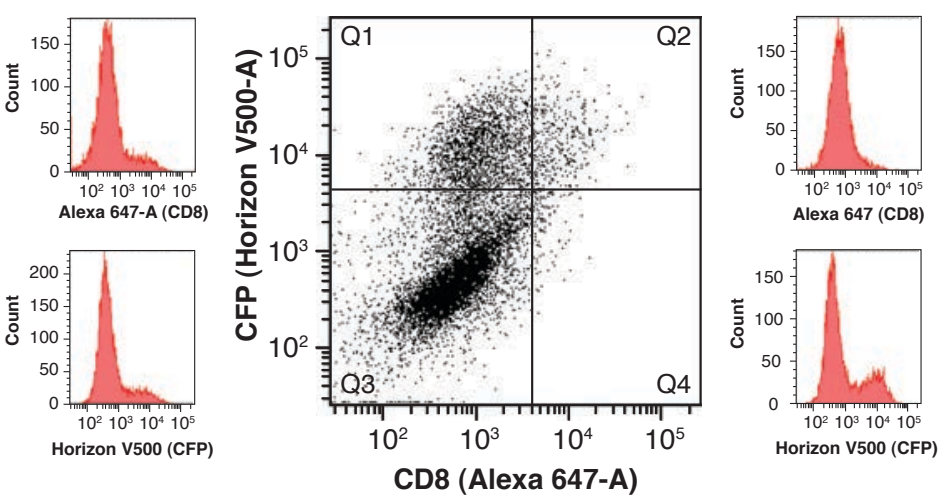

D
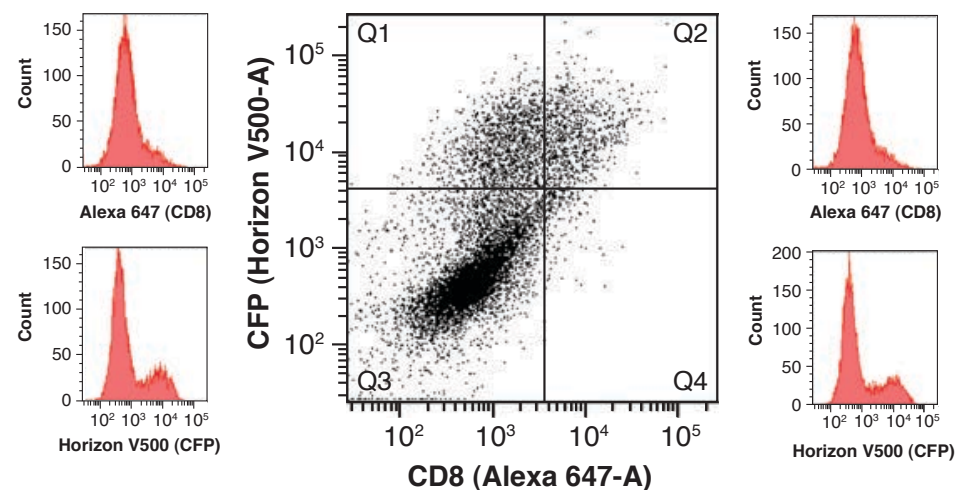

$\mathbf{F}$

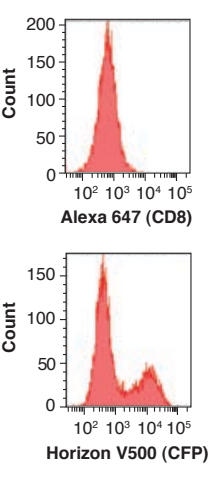

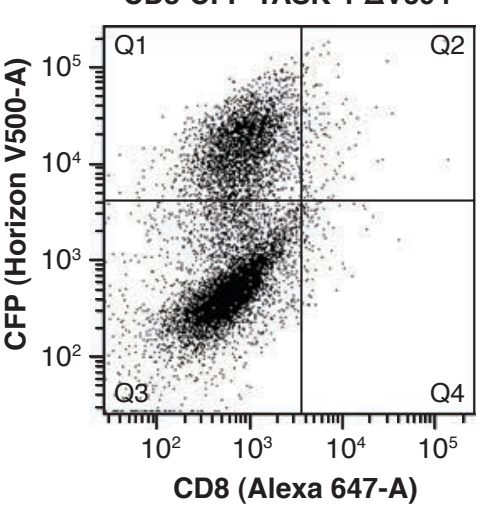
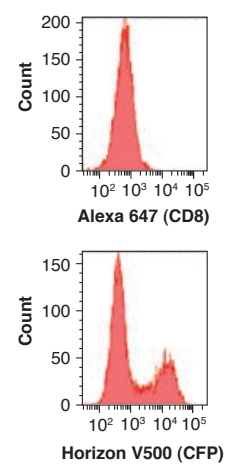

Figure 24: Flow cytometry assays to determine the effect of replacing S392, or S393 by Alanine on cell surface expression of the reporter-protein. Reporter protein assays reveal a potential regulatory role of S392 in TASK-1 protein trafficking. Cos7 cells were transiently transfected with DNA encoding TASK-1-derived reporter proteins. Surface localized proteins were detected employing a monoclonal anti-CD8 primary antibody and an Alexa-647 conjugated secondary antibody. Following detection, cells were subjected to Flow cytometry and the relative cell surface expression of different reporter proteins was determined. Displayed are representative dot-plots and 
histograms for each reporter protein employed. As described for TASK-3-derived reporter-proteins, the relative cell surface expression was expressed as a ratio of the mean of the CD8 signal with the mean of the CFP signal in CFP-positive cells. All values were normalized to the TASK-3 wild-type construct.

Comparing CD8-CFP-TASK-3 WT and CD8-CFP-TASK-1 WT, I observed a reduction in cell surface expression of the TASK-1 reporter protein by threefold (Figure 25, Compare $1^{\text {st }}$ and $2^{\text {nd }}$ Column). Replacement of S392 with alanine led to an increase in cell surface expression by almost three-fold, indicating an inhibitory role of $\mathbf{S} 392$ on cell surface expression in vivo (Figure 25, $3^{\text {rd }}$ Column). Disruption of COPI binding by mutation of K389, also led to an increase in relative cell surface expression by three-fold, to levels comparable to CD8-CFP-TASK-3 WT (Figure 25, $4^{\text {th }}$ Column). This finding is in agreement with findings reported by Zuzarte et al. 2009 (11) showing that mutation of this lysine residue removes the necessity of 14-3-3 to bind to the C-terminus, allowing the reporter-protein to reach the surface in an 14-3-3COPI independent manner. On the other hand, mutation of S393 led to a decrease in cell surface expression of the reporter protein by five-fold compared to CD8-CFP-TASK-3 WT (two-fold compared to TASK-1 WT), strongly supporting the hypothesis, that transient phosphorylation of S392 is sufficient to inhibit 14-3-3 binding, thereby inhibiting cell surface expression of the reporter protein, retaining the protein in early compartments of the secretory pathway (Figure 25, Compare $1^{\text {st }}$ and $5^{\text {th }}$ Column). Additionally, I assessed the relative cell surface expression of a truncated version of the TASK-1 reporter-protein. Consistent with current literature (11), a significant decrease in relative cell surface expression of the reporter protein compared to the TASK-3 wild-type reporter-protein was observed. Figure 25 illustrates our findings, allowing a direct comparison of all reporter proteins employed. 


\section{Results}

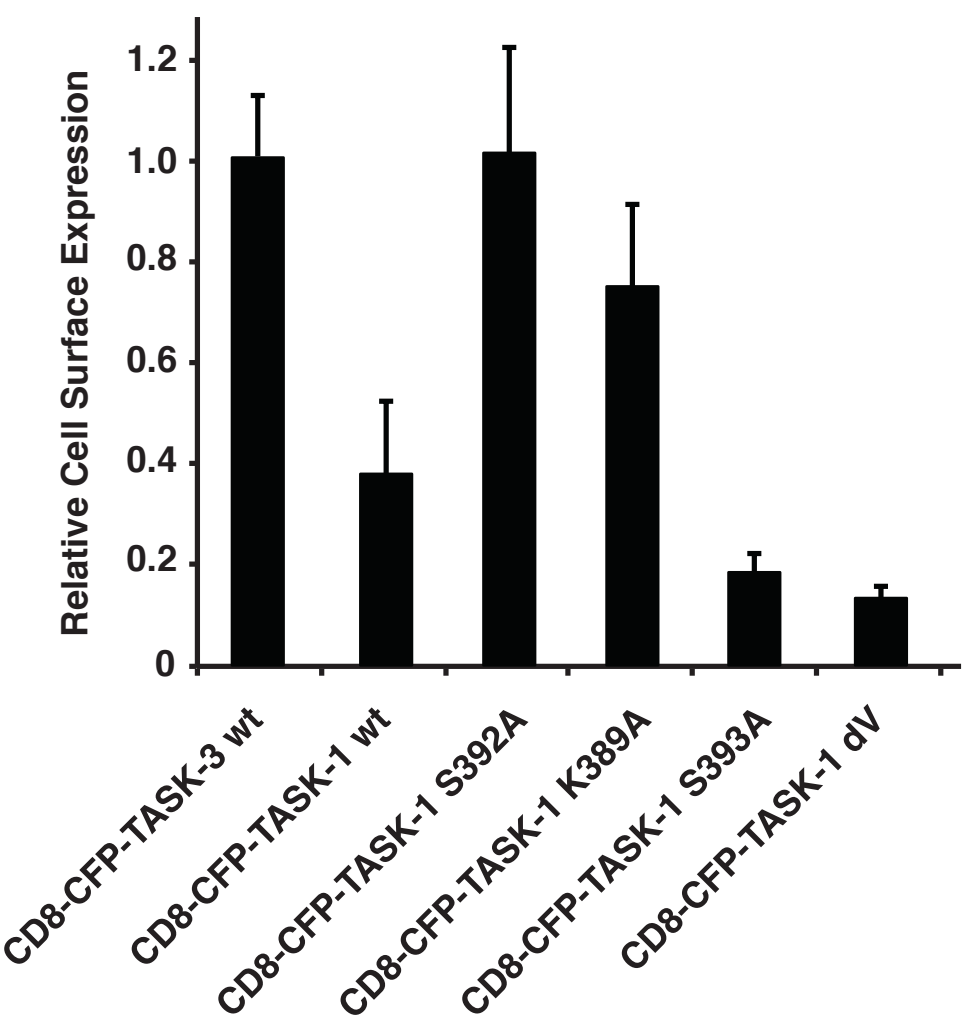

Figure 25: Relative cell surface expression of all TASK1-derived reporter proteins normalized to CD8-CFP-TASK3 WT construct. Mutation of S392 led to a significant increase in cell surface expression of the CD8-TASK-1 reporter-protein, confirming a potential regulatory role of S392 in TASK-1 trafficking. The mean signal intensity of the anti-CD8 signal (Alexa 647) was expressed as a ratio with the mean signal intensity of the CFP signal (Horizon V500) in CFP-positive cells and normalized to the wild type TASK-3 construct. Cells were transfected in six independent experiments, error bars depict standard error of the mean (s.e.m.).

Transient phosphorylation of S392 reduces the relative cell surface expression of TASK-1-derived reporter proteins

We further assessed the phosphorylation state of each TASK-1-derived reporter-protein in vivo. Samples were prepared as described for TASK-3 derived reporter-proteins and subsequently resolved on Phostag and standard SDS-PAGE gels. Gels were westernblotted and proteins were detected with a CD8-a-antibody and a fluorescently labeled secondary antibody. 

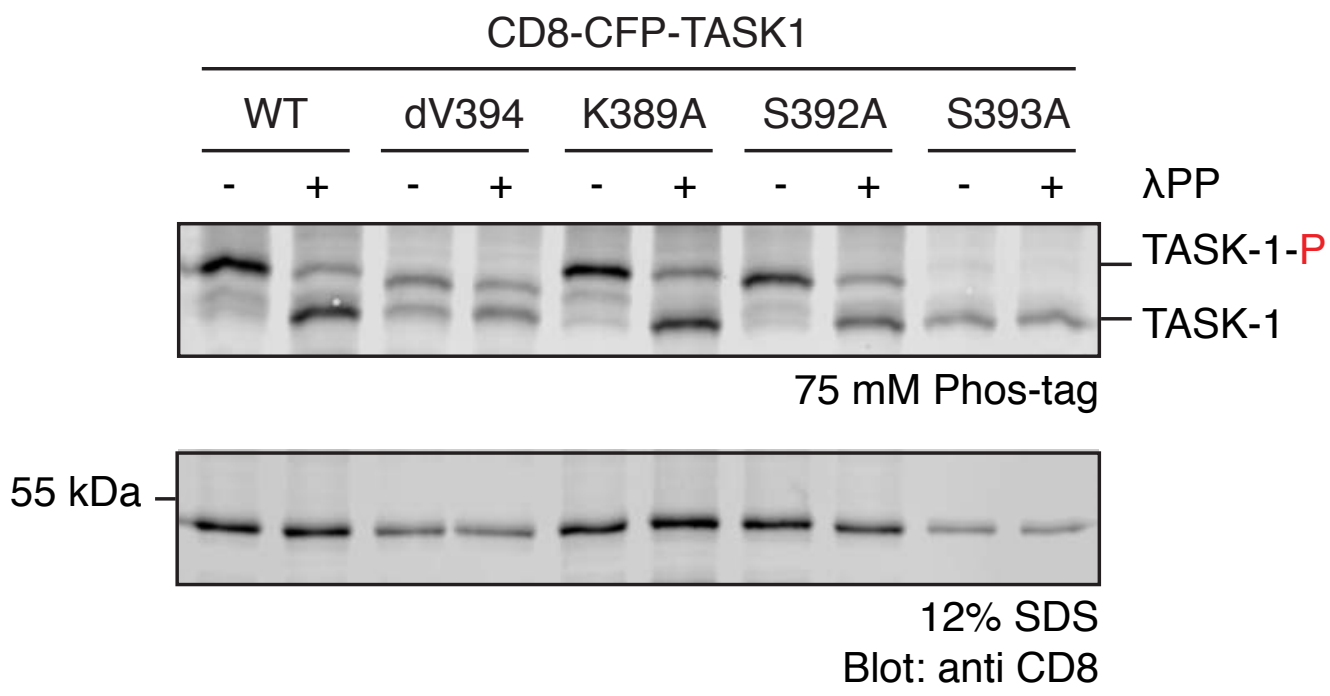

Figure 26: In vivo phosphorylation of indicated TASK-1-derived CD8-reporterproteins. Membrane protein samples were incubated in presence and absence of $\lambda$ protein phosphatase ( $\lambda$ PPase) and subsequently separated on either standard SDSPAGE, or Phostag SDS-PAGE gels. Gels were westernblotted and proteins were detected with a CD8-a-antibody and a fluorescently labeled secondary antibody. Phosphorylation bands are indicated by the upward band shift relative to samples treated with $\lambda$ PPase. The blot is representative for seven independent transfections. TASK-1: unphosphorylated TASK-1-derived reporter-protein; TASK-1-P: single phosphorylated TASK-1-derived reporter-protein.

Similar to CD8-CFP-TASK-3 WT (Figure 23, Lane 1 and 2) one phosphorylation band, indicated by the upward band shift relative to samples treated with $\lambda$ PPase, was observed for CD8-CFP-TASK-1 WT (Figure 26, Lane 1). Treatment with $\lambda P P a s e$ altered the migratory behavior of the reporter protein (Figure 26, Lane 2) to a faster migrating protein species. Similar observations were made for CD8-CFP-TASK-1 K389A and CD8-CFP-TASK-1 S392A, indicating that the protein was efficiently phosphorylated by cellular kinases (Figure 26, Lane 5 and Lane 7). The migratory behavior of each reporter protein was altered after treatment with $\lambda$ PPase, indicating efficient dephosphorylation of the reporter-protein (Figure 26, Lane 6 and Lane 8). For 


\section{Results}

CD8-CFP-TASK-1 S393A a faint band corresponding to the phosphorylated reporter protein was observed (Figure 26, Lane 9) confirming that the reporter protein was 'transiently' phosphorylated in vivo. This band disappeared after treatment with $\lambda$ PPase, demonstrating that this band corresponded to the phosphorylated reporter-protein. Similar to the phosphorylation state of CD8CFP-TASK-3 $\Delta$ V374 only a fraction of CD8-CFP-TASK-1 $\Delta$ V394 reporter protein was phosphorylated in vivo (Figure 26, Lane 3). Two distinct protein bands were observed, corresponding to the phosphorylated (upper band) and unphosphorylted (lower band) reporter protein. Treatment with $\lambda$ PPase partially altered the migratory behavior of the protein (Figure 26, Lane 4), suggesting that the protein was inefficiently dephosphorylated by $\lambda$ PPase due to reasons unknown.

Strikingly, I observed only one phosphorylation band for CD8-CFP-TASK-1 WT, which corresponded to the single phosphorylated reporter-protein, when the migratory behavior is compared to CD8-CFP-TASK-1 K389A, CD8-CFPTASK-1 S392A and CD8-CFP-TASK-1 S393A. From these observations I conclude that S393 is efficiently phosphorylated by cellular kinases, and possibly masked form cellular phosphatase by binding of 14-3-3 proteins (117). I observed a minor band reflecting the phosphorylated species of CD8CFP-TASK-1 S393A, indicating inefficient phosphorylation by cellular kinases, or efficient dephosphorylation by cellular phosphatases. 


\section{Phosphorylation of TASK-1 and TASK-3 C-termini impairs COPI binding}

Protein trafficking of TASK-1 and TASK-3 involves two key regulators, by which the relative amount of protein expressed at the cell surface is determined. One of these key regulators is COPI. COPI-binding retrieves the channel to early compartments of the secretory pathway. Interactions with COPI are mediated by an ER retention-retrieval motif present at the distal TASK-1 and TASK-3 C-terminus (KRR). The second key regulator facilitating anterograde protein trafficking is 14-3-3. Upon phosphorylation of S393 in TASK-1 and S373 in TASK-3, a conserved serine residue of a mode III 14-33-binding motif, 14-3-3-binding masks the adjacent ER-retention-retrieval motif, by sterically occluding COPI-interactions. In general, binding of 14-3-3 or COPI was considered to be mutually exclusive. So far COPI binding to the trafficking control region of TASK-1 and TASK-3 has been evaluated in presence of other potential interaction partners, using cellular fractions. To evaluate the functional consequence of phosphorylation of either S373 in TASK-3, or S392/S393 in TASK-1 on COPI binding we exploited previous findings, demonstrating that the ER retention-retrieval motif presented by TASK-1 and TASK-3 (KRR) can also be recognized by the yeast COPI coat. Therefore COPI coat was purified from yeast (purifications performed by Dr. Eric Arakel) and the binding to constructs presenting either the distal TASK-1, or TASK-3 C-terminus (C15) replacing the C-terminal KKXX ER-retention signal of the cytoplasmic tail of the yeast protein MST-27, fused to the GSTprotein was studied. The MST27-linker increased the accessibility of COPI to the TASK C-terminal peptide, extensively characterized by several COPI binding experiments $(10,112)$.

GST-MST27-TASK-1 and GST-MST27-TASK-3 fusion proteins and the GSTprotein-tag were recombinantly expressed in and affinity purified from E.coli. Since they were expressed in bacteria, fusion-proteins were not phosphorylated. GST-MST27 fusion proteins were incubated with PKA in presence and absence of ATP, as previously described for GST-TASK fusionproteins. Each protein was phosphorylated for 12 hours at $4^{\circ} \mathrm{C}$. To avoid 


\section{Results}

further degradation of the protein, as observed in Figure 27 (lower panel, Lane $4-6)$, every buffer used was supplemented with protease inhibitors (PMSF and aPMSF). Following phosphorylation fusion-proteins were incubated with affinity purified COPI coat. Following separation, gels were blotted and proteins were detected using a specific COPI coat antibody (T270, kindly provided by Anne Spang). The total amount of GST or GST-fusion protein loaded was assessed after samples were separated on a standard SDS-PAGE gel and protein bands were stained with Coomassie solution. We further quantified the amount of COPI coat bound to the GST-MST27-fusion proteins treated with PKA in absence and presence of ATP (EM: Energy mix (108)).

A

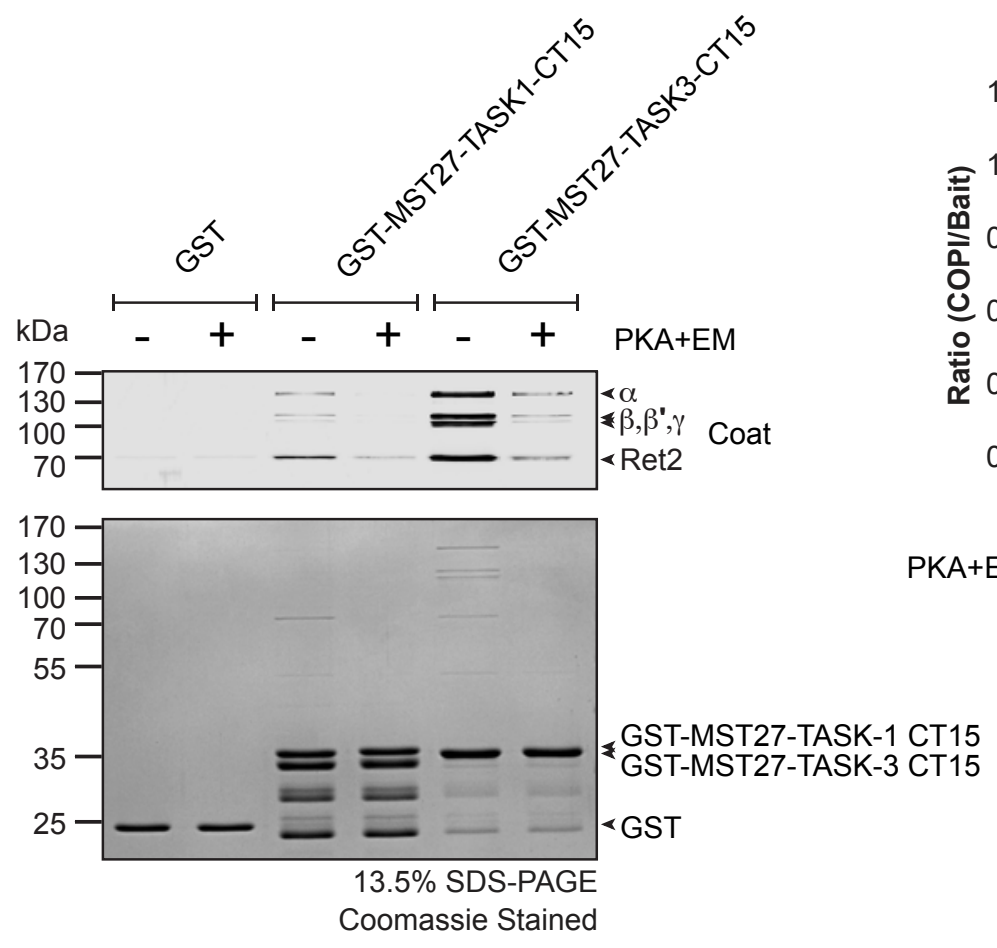

B

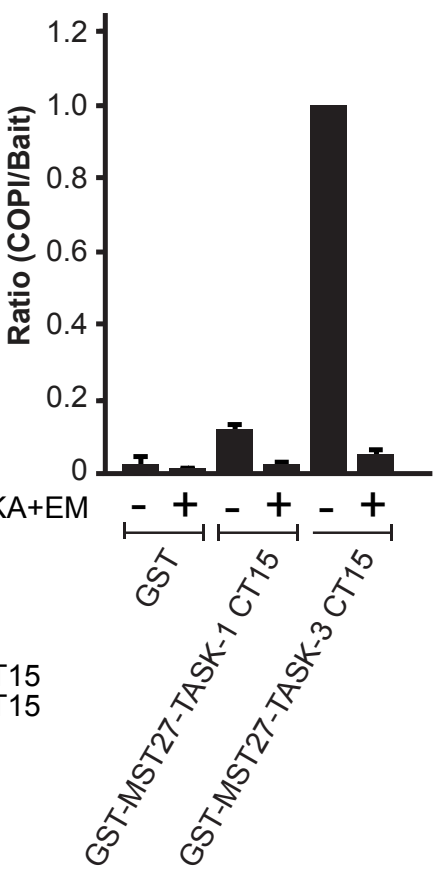

Figure 27: Phosphorylation of TASK-C-termini disrupts COPI binding. Pull-down experiments were performed employing either GST, GST-MST27-TASK-1 or GSTMST27-TASK-3 proteins. Proteins were incubated with PKA in absence or presence of ATP prior to incubation with purified yeast COPI coat. Samples were separated on standard SDS-PAGE gels and subsequently stained with Coomassie solution to visualize the bait. The amount of COPI coat was determined by Western-blotting, 
utilizing a specific COPI coat antibody, with which several subunits of the yeast COPI coat were detected. A: representative Western-blot showing different amounts of COPI coat bound to GST-MST27 fusion-proteins treated with PKA in absence and presence of energy mix (EM). Control incubations with GST are shown in Lane 1 and Lane 2. B: Quantification of A, forming the ratio of COPI protein detected (the ratio of all subunits of the COPI vesicular coat complex, indicated above) with the total amount of protein loaded (Bait). The experiment was repeated three-times. Error depicts standard error of the mean (s.e.m.). The experiment was carried out with help of Dr. Eric Arakel.

We observed that a substantial amount of COPI coat bound to the TASK-3 WT protein (Figure 27, A: Lane 5, B: $5^{\text {th }}$ Column), confirming observations made by O'Kelly et al., 2002 and Zuzarte et al., $2009(11,18)$. The amount of COPI coat bound was significantly decreased after phosphorylation of the bait protein by PKA (Figure 27, A: Lane $6, \mathrm{~B}: 6^{\text {th }}$ Column) indicating that phosphorylation is sufficient to disrupt COPI binding. Similar observations were made for GST-MST27-TASK-1 fusion proteins incubated with PKA in absence and presence of ATP. The total amount of COPI coat bound to the unphosphorylated TASK-1 fusion-protein was reduced by approximately fivefold, indicating that the presentation of a second serine residue (S392) present in TASK-1 also affects COPI binding. After phosphorylation of the GST-MST27-TASK-1 fusion protein, COPI coat binding was reduced to levels similar to control experiments performed with the GST-protein tag on its own. GST essentially did not bind the COPI coat. These observations clearly demonstrate that phosphorylation of the TASK-1 or TASK-3 C-terminus is sufficient to impair COPI-binding. We further conclude that 14-3-3 proteins and COPI do not bind the TASK-1 or TASK-3 C-terminus in a mutually exclusive way, suggesting that the binding equilibrium rather exist between the phosphorylated (14-3-3 binding) and unphosphosphorylated state of the protein (COPI-binding), coupled by the action of kinases and phosphatases. 


\section{Results}




\section{Discussion}

\section{Small sequence differences in TASK-3 and TASK-1 cause biological relevant changes in 14-3-3 binding}

It is well established that 14-3-3 proteins bind the C-terminus of TASK-1 and TASK-3 in a phosphorylation-dependent manner, thereby releasing the channel from ER retention by preventing COPI-binding to an ER retentionretrieval motif (KRR) presented by both C-termini $(1,3,11,17,18,64)$. The cell surface expression of these channels is thought to be controlled by mutually exclusive binding of either the COPI vesicle coat, or 14-3-3 proteins $(1,3,18)$. Thus, the function of this trafficking control region is based on competitive binding of both interaction partners (1).

In 2002, a study by Rajan et al. (2002) reported that different $14-3-3$ isoforms interact with a trafficking motif present at the distal C-terminus of TASK-1, TASK-3 and TASK-5 (17). By screening yeast two-hybrid libraries from rat brain, rat hippocampus and rat embryo using the full-length C-terminus of TASK-1, TASK-3 and TASK-5 four individual 14-3-3 isoforms were identified (17). Yeast two-hybrid analysis showed that all mammalian 14-3-3 isoforms could interact with a trafficking control motif present in the distal C-terminus of each channel, RRxS/Tx. According to their observations interaction with 14-33 proteins promoted functional expression of the channel at the cell surface (17).

In parallel, O'Kelly et al. (2002) found that 14-3-3ß interacts with the last 16 amino acids of TASK-1. Utilizing yeast two-hybrid (from a heart cDNA library) and in vitro pull-down experiments, which were performed with cellular extracts, then demonstrated direct interactions of 14-3-3 proteins and TASK-1 C-terminal peptides. They proposed a model, by which mutually exclusive binding of either the COPI vesicle coat, or 14-3-3 regulate the amount of protein expressed at the cell surface (18). Binding of COPI was thought to be 


\section{Discussion}

mediated by a N-terminal dibasic ER retention motif, whereas 14-3-3 bound to a non-canonical 14-3-3 binding motif located at the distal C-terminus (18).

Both reports elucidate the role of 14-3-3 binding in protein trafficking along the secretory pathway and demonstrate that proteins unable to associate with 143-3 failed to reach the surface efficiently. The non-canonical 14-3-3 binding motif found at the C-terminus of TASK-1 and TASK-3 was subsequently described as a mode III 14-3-3 binding motif, which operates depending on phosphorylation of a conserved serine/threonine residue, $\mathrm{RXpS} / \mathrm{pT}\left(\mathrm{X}_{1-2}\right)$ $\mathrm{COOH}(14,116)$.

A subsequent study by Zuzarte et al. (2009) demonstrated by systematic mutation of the TASK-1 C-terminus that interactions with COPI are mediated by a C-terminal, tri-basic ER retention retrieval signal (KRR) adjacent to the conserved serine of the mode III 14-3-3 binding motif $(14,116)$, rather than a dibasic $\mathrm{N}$-terminal retrieval and retention motif (18). The role of this trafficking control region was further emphasized using TASK-1 and TASK-3-derived reporter-proteins, showing that the cell surface expression of the channel/reporter protein is highly dependent on their ability to associate with 14-3-3 proteins. 14-3-3 binding deficient reporter/channel proteins failed to reach the cell surface efficiently $(11,17,18)$. Based on a previous report, Zuzarte et al. (2009) highlighted that TASK-3 reaches the cell surface more efficiently than TASK-1, indicative of a different mechanism regulating TASK-1 transport to the plasma membrane $(11,66)$.

Up until recently, the molecular details of 14-3-3 binding to the trafficking control region of TASK-1 and TASK-3 were unknown. Anders et al. (2013) reported a crystal structure that emphasized intricate interactions formed between the phosphorylated TASK-3 C-terminus and multiple residues on the inner sider of the 14-3-3 $\sigma$ binding groove and additionally determined the binding parameters of this particular interaction (107). 
Striking differences can be observed comparing the amino acid residues flanking the ER retention-retrieval motif, which precede the conserved serine residue of the mode III 14-3-3 binding motif at the distal C-terminus of TASK-1 and TASK-3. TASK-3 features an additional lysine residue (K372), whereas TASK-1 presents a second serine residue (S392) that could potentially be phosphorylated in vivo. In this thesis I investigated interactions between TASK-C-termini and 14-3-3 proteins, as well as COPI quantitatively and present evidence that small sequence differences cause biologically relevant changes in 14-3-3 and COPI binding.

To gain quantitative insight into the mechanisms, by which these trafficking control regions act, I first determined the binding parameters of all seven mammalian 14-3-3 isoforms to TASK-3 derived C-terminal peptides phosphorylated at S373 and TASK-1 derived C-terminal peptides phosphorylated at S393, quantitatively. Both residues correspond to the conserved serine residue of the mode III 14-3-3 binding motif $(14,116)$. Interestingly binding affinities determined for TASK-3 WT pS373 were two orders of magnitude higher than affinities determined for TASK-1 WT pS393, with dissociation constants varying between $100 \mathrm{nM}$ for 14-3-3n - TASK-3 WT pS373 and $10 \mu \mathrm{M}$ for $14-3-3 \eta$ - TASK-1 WT pS393. These findings illustrate that the numerous published pull-down and yeast two-hybrid experiments performed effectively operate down to $K_{D}$ values of $10 \mu \mathrm{M}$, obscuring substantial affinity differences between isoforms due to their qualitative nature $(11,17,18)$. I further determined the binding affinity of 14-3$3 \sigma$ to TASK-3 WT pS373 with a $K_{D}$ value of $3.6 \pm 0.4 \mu \mathrm{M}$. This value perfectly matches a value reported by Anders et al., 2013, with a $K_{D}$ value of $4.1 \pm 0.8 \mu \mathrm{M}$ for interactions measured between a TASK-3-derived hexapeptide and $14-3-3 \sigma$ by isothermal titration calorimetry (ITC). Affinity differences between TASK-1 and TASK-3 are in perfect agreement with a crystal structure by Anders et al., highlighting distinct hydrogen bonds formed between K372 in TASK-3 and D225 and N226 on the inner side of the 14-3$3 \sigma$ binding groove (107). Hydrogen bonds formed by these residues might 


\section{Discussion}

contribute substantially to the high affinity binding of 14-3-3 proteins to the phosphorylated TASK-3 C-terminus. I reason that a serine, or phosphoserine residue at a position equivalent to $\mathrm{K} 372$ would potentially destabilize interactions between the C-terminal peptide and 14-3-3 proteins. And this is exactly what I observed for TASK-1 WT pS393, TASK-1 WT pS392 and TASK-1 WT pS392pS393 (Figure 11, Figure 12, Figure 13 and Table 11). Affinities determined for interactions between 14-3-3 proteins and either TASK-1-derived peptide were substantially lower than for TASK-3 WT pS373.

\section{Phosphorylation of S392 in TASK-1 impairs COPI and 14-3-3 binding}

It is well established that trafficking control motifs consisting of multiple basic residues are involved in COPI-mediated retention and retrieval of membrane proteins, such as membrane receptors and ion channels.

Because the exact binding site of the trafficking control region of TASK-1 and TASK-3 in COPI is unknown, a potential contribution of K372 in TASK-3 or S392 in TASK-1 to COPI binding affinity could not be addressed quantitatively. In general interactions with COPI involve multiple basic residues, e.g. $\operatorname{RKXX}(8), \operatorname{KKXX}(9), \operatorname{RXR}(10)$ and $\operatorname{KRR}(11)$. Therefore, we predict that one additional lysine residue in TASK-3 will contribute significantly to COPI binding, compared to S392 in TASK-1.

We tested this hypothesis and conducted pull-down experiments with GSTMST27-TASK-1 WT, GST-MST27-TASK-3 WT fusion proteins and purified yeast COPI coat. We exploited a previous finding that the ER retentionretrieval motif of TASK-1 and TASK-3 can also be recognized by yeast COPI coat (11), therefore pull-down experiments performed with TASK-1 and TASK3-derived fusion-proteins and purified yeast COPI coat should reflect biological relevant interactions. In addition, we assessed the functional consequence of phosphorylation of the TASK-1 and TASK-3 C-terminus on COPI binding. 
Interestingly, the amount of protein co-purified with unphosphorylated TASK-3 fusion protein was approximately five-fold higher than the amount of protein co-purified with TASK-1 derived fusion proteins (Figure $27 \mathbf{A}$ and $\mathbf{B}, 3^{\text {rd }}$ and $5^{\text {th }}$ lane). These results perfectly match our predictions and demonstrate that small sequence differences cause a biological/physiologically relevant change in COPI binding, in addition to the effects on 14-3-3 binding that I uncovered. We were even more intrigued by a substantial decrease in the amount of COPI coat co-purified with phosphorylated TASK-1 and TASK-3 fusion protein. These observations suggest that phosphorylation is sufficient to disrupt COPI binding and facilitate release of the protein from ER retention and retrieval.

Phosphorylation of S392 in TASK-1 perturbed both 14-3-3 and COPI-binding to TASK-1 derived C-terminal peptides. These observations are reminiscent of results reported by Arakel et el., demonstrating that ATP sensitive potassium channels $\left(\mathrm{K}_{\text {ATP }}\right)$ can reach the cell surface in a 14-3-3 and COPI independent manner, upon phosphorylation of a serine residue flanking a tribasic ER retention and retrieval motif (6).

Arakel et al. investigated the functional expression of ATP sensitive potassium channels $\left(\mathrm{K}_{\mathrm{ATP}}\right)$ in cardiac tissue, emphasizing the role of 14-3-3 and COPI in the cell surface expression of the channel. Furthermore this study provides detailed insight into the tissue-specific expression of 14-3-3 proteins, demonstrating that, although ubiquitously expressed, the amount of available 14-3-3 may be restricted (6). In general, $K_{\text {ATP }}$ channels exist as heterooctamers formed by four pore-forming Kir6.1, or Kir6.2 subunits and four sulfonylurea (SUR1 or SUR2) subunits (118). Individual subunits and intermediary complexes exposing arginine-based COPI sorting motifs are retained at the ER until stoichiometrically assembled hetero-octamers are formed. In fully assembled channels, the tribasic ER retention and retrieval motifs (RKR) in Kir6.2 and SUR1 are masked by individual subunits allowing progression along the secretory pathway $(16,119)$. 


\section{Discussion}

Based on a previous report by Heusser et al. (and others) demonstrated that 14-3-3 proteins associate with Kir6.2 potassium channels in vitro and in vivo, promoting the functional expression of $\mathrm{K}_{\text {ATP }}$ channels to the cell surface (120). 14-3-3 binding is thought to override ER retention by masking the RKR trafficking control motif, present in each Kir6.2 and SUR1 subunit, when presented as a di- or tetramer (108, 120-123). In case of Kir6.2, association with 14-3-3 proteins facilitates the regulated release of the channel from COPI-mediated ER retention and thereby promoting the functional expression of the channel at the cell surface.

According to Arakel et al. phosphorylation of a serine residue preceding the ER retrieval motif uncouples the cell surface transport of $\mathrm{K}_{\mathrm{ATP}}$ channels, such as Kir6.2, from the antagonistic action of 14-3-3 and COPI, releasing stored pools of Golgi-retained $\mathrm{K}_{\mathrm{ATP}}$ in order to respond to external stimuli, such as $\beta$ adrenergic stimulation.

Comparable to TASK-1 the presence of 14-3-3 is required to facilitate efficient cell surface transport of $\mathrm{K}_{\mathrm{ATP}}$ channels. In cells, such as cardiac myocytes the amount of available ('free') 14-3-3 is limited and might therefore require an alternative mechanism facilitating the release of these potassium channels from the Golgi to respond to external stimuli (6).

Two well characterized cardiac potassium channels are Kir6.2 and TASK-1 (2). It would also be reasonable to consider a similar mechanism controlling the cell surface expression of both channels that maintains the electrical excitability of these cells, just like Kir6.2. The trafficking control region of TASK-1 might lead to an accumulation of preassembled TASK-1 channels in the Golgi that can be released upon $\beta$-adrenergic stimulation and concomitant phosphorylation. In this situation, double phosphorylation of the TASK-1 trafficking control region could trigger the 14-3-3 and COPI independent release of a pool of Golgi-stored/retained TASK-1 channels. 
A previous report by Renigunta et al. demonstrated that TASK-1 interacts with another adaptor protein that retains the channel in compartments of the early secretory pathway, by exposure of a secondary ER retrieval motif present at its C-terminus - p11 (also denoted as S100A10). Interactions are mediated by a 40 amino acid long region in TASK-1, which is located upstream of the mode III 14-3-3 binding motif (66). The recruitment of a cytosolic retention factor represents another possibility to modulate the cell surface expression of TASK-1. Retention of the channel by $\mathrm{p} 11$ might thereby represent another mechanism by which an accumulation of TASK-1 in the Golgi could be achieved. Furthermore the substantially lower affinity of the TASK-1 trafficking control region for COPI might potentially be compensated by recruitment of p11. The higher affinity of the TASK-3 trafficking control region can be explained by the presence of a lysine residue flanking the ER retention and retrieval signal, thereby generating a dibasic ER retention and retrieval motif, $\operatorname{RKXX}(8)$.

\section{Quantitative evaluation of two distinct mutations of TASK-1 and TASK-3 thought to abolish 14-3-3 binding}

Two commonly and interchangeably used manipulations to disrupt 14-3-3 binding, in order to study the role of 14-3-3 in TASK channel trafficking, are:

1) deletion of the distal valine residue of TASK-1 or TASK-3, and 2) mutation of the conserved serine residue of the mode III 14-3-3 binding motif $(1,3,11$, $17,18)$.

O'Kelly et al. investigated 14-3-3 binding to C-terminal variants of TASK-1 by in vitro pull-down experiments from cellular lysates, Rajan et al. employed an alternative approach to investigate 14-3-3 binding to different TASK-derived C-termini (TASK-1, TASK-3 and TASK-5) - Yeast two hybrid analysis (17, 18). Both approaches assess interactions between 14-3-3 and different TASK-derived client proteins qualitatively and obscure 14-3-3 isoform specific differences. Qualitatively, both methods failed to capture interactions of 14-3-3 


\section{Discussion}

proteins with 14-3-3 binding deficient (TASK-1 S393A, TASK-3 S373A) and truncated TASK-derived substrates (TASK-1 $\Delta$ V394, TASK-3 $\Delta 374$ ).

I quantitatively compared 14-3-3 binding parameters obtained by fluorescence polarization titration (FP), employing 14-3-3 binding deficient C-terminal peptides (TASK-3 S373A and TASK-1 S393A) and a truncated version of each C-terminus (TASK-3 pS373 $\Delta$ V374 and TASK-1 pS393 $\Delta$ V394). And indeed, for peptides, in which the 14-3-3 binding relevant serine that is part of the mode III 14-3-3 binding motif was replaced with alanine, no 14-3-3 binding was observed. These findings are in agreement with current literature $(11,17$, 18), demonstrating that a phosphoserine is required to bind 14-3-3 proteins with high affinity.

Interestingly, 14-3-3 binding to the truncated TASK-1 C-terminus was lost, which is in agreement with previous reports $(11,17,18)$, but partially sustained for the truncated TASK-3 C-terminus. Since phosphorylation is essential to detect any 14-3-3 binding, truncated peptides were constitutively phosphorylated at S373 and S393, respectively. These observations indicate that both commonly used manipulations have a distinct effect on 14-3-3 binding, differences between TASK-1 and TASK-3 derived mutants might again be explained by small sequence differences causing a biological relevant effects. The presence of a second serine residue might here as well destabilize interactions between TASK-1 pS393 $\Delta$ V394 peptides and 14-3-3 proteins, whereas the presence of K372 in TASK-3 might partially 'rescue' 143-3 binding.

Interestingly, binding affinities determined for TASK-3 pS373 $\Delta$ V374 were substantially higher than binding affinities determined for TASK-1 WT pS393. Considering that TASK-1 reaches the cell surface more efficiently than TASK3 pS373 $\triangle \mathrm{V} 374$, the loss of $14-3-3$ binding to the truncated TASK-3 Cterminus cannot satisfactorily explain the biological outcome of this manipulation - accumulation of the reporter protein in internal cellular 
compartments - as previously reported by various other workgroups $(11,17$, 18, 113).

In context of a phosphorylated mode III 14-3-3 binding motif and in line with a previously reported structure of $14-3-3 \sigma$ associated with a TASK-3-derived hexapeptide (107), it was reported that the carboxyl C-terminus of TASK-3 forms only one hydrogen bond with one lysine residue on the inner side of 14$3-3 \sigma$ (K122), whereas more intricate interactions are formed by the phosphate group with residues on the inner side of the 14-3-3 binding groove (R56, R129, Y130, E182). Here I provide evidence that the distal valine residue present in TASK-1 and TASK-3 does not substantially contribute to the observed binding affinities determined for TASK-3 WT pS373 or TASK-1 WT pS393.

In summary, I demonstrate that the two commonly and interchangeably used manipulations have distinct effects on 14-3-3 binding. Whereas mutation of the conserved serine residue of the mode III 14-3-3 binding motif to alanine in TASK-3 abolished 14-3-3 binding, deletion of the C-terminal valine residue of TASK-3 did not.

\section{Differences in cell surface expression of TASK-1 and TASK-3 derived reporter-proteins reflect differences in 14-3-3 and COPI binding}

A report by Zuzarte et al. illustrated that the mechanism underlying the change of cell surface expression of TASK-1 or TASK-3 could be investigated employing CD8-based reporter proteins, exposing the full-length $\mathrm{C}$-termini of TASK-1 and TASK-3 channels (11). I exploited these findings and generated CD8-based reporter proteins to study the relevance of different 14-3-3 and COPI binding affinities to different TASK trafficking control regions in vivo. To directly compare my in vitro and in vivo findings I generated constructs exposing the last 15 amino acids of the TASK-1 and the TASK-3 C-terminus. Additionally, insertion of a fluorescence probe/fluorophore (CFP) as a linker between CD8 and the TASK C-terminal peptides was particularly useful to 


\section{Discussion}

identify cells expressing the reporter-protein without relying on antibody detection.

I found that the marked differences in 14-3-3 and COPI binding affinities between TASK-1 and TASK-3 are also relevant in vivo. I demonstrated that the TASK-3 WT reporter protein reached the cell surface efficiently, and was mainly localized at the plasma membrane (Figure 21 and Figure 22), whereas the TASK-1 reporter protein reached the cell surface less efficiently and was largely retained in intracellular compartments (Figure 24, A and B, Figure 25). These findings are in agreement with observations previously reported by Renigunta et al., showing that TASK-3 is more efficiently transported to the cell surface than TASK-1, while investigating the effect of p11 binding on retention of TASK-1 (66).

Furthermore, I illustrated the importance of S392 present in TASK-1 on cell surface expression of the reporter protein. Mutation of S392 to alanine led to a significant increase in cell surface expression of the reporter protein. I interpret this effect due to increased 14-3-3 binding affinities. Upon mutation, S392, which when phosphorylated leads to a strong decrease in 14-3-3 binding affinity, can no longer be phosphorylated by cellular kinases and is expected to recruit 14-3-3 proteins more efficiently (prerequisite: phosphorylation of S393 by PKA). And indeed, I observed comparable levels of cell surface expression of CD8-CFP-TASK-1 S392A and CD8-CFP-TASK-3 WT (Table 10, Figure 24 C, Figure 25). The same effect was observed upon mutation of the ER retention and retrieval motif by replacing K389 with alanine $(11,113)$. Although, both serine residues are present in this reporter protein and accessible to cellular kinases, in the absence of a functional COPIinteraction motif, 14-3-3 binding to the TASK-1 C-terminus is no longer required for cell surface expression of the reporter protein, therefore the reporter protein reaches the cell surface efficiently, in a 14-3-3 and COPIindependent manner (11). The reduced cell surface expression of the TASK-1 WT construct (CD8-FP-TASK-1 WT) with respect to the change in cell surface 
expression of TASK-1 S392A and TASK-1 K369A is consistent with the rational that both interaction partners, COPI and 14-3-3 proteins, can access the trafficking control region in the steady state. My observations also demonstrate that transient phosphorylation of S392 reduces the efficacy by which 14-3-3 can remove the cargo protein from COPI-mediated ER retention.

Additional experiments carried out with truncated ( $\triangle$ V 374 : TASK-3 and $\triangle$ V394: TASK-1) and 14-3-3 binding deficient reporter proteins (S373A: TASK-3 and S393A: TASK-1) allowed me to assess the biological outcome of both manipulations in vivo. I observed that truncated as well as 14-3-3 binding deficient reporter proteins failed to reach the cell surface efficiently and accumulated partially in COPI positive structures (Kilisch et al., 2016). Although both manipulations have a similar biological outcome, the lack of 143-3 binding can only explain the observations made for 14-3-3-binding deficient reporter-proteins CD8-CFP-TASK-3 S373A and CD8-CFP-TASK-1 S393A (Figure 21, B; Figure 22, $4^{\text {th }}$ Column; Figure 24, E; Figure 25, $5^{\text {th }}$ Column). A quantitative analysis of 14-3-3 binding to truncated versions of the TASK-3 and TASK-1 C-terminus demonstrated that 14-3-3 proteins associated with the truncated TASK-3 C-terminus (TASK-3 pS373 $\Delta$ V374) with calculated binding affinities in the low $\mu \mathrm{M}$ range, however 14-3-3 binding to the truncated TASK-1 C-terminus was lost. In summary, I confirmed the role of 14-3-3 proteins in cell surface expression of TASK-1 and TASK-3 derived reporter proteins $(11,17,18)$, I further demonstrated that two commonly used manipulations with similar biological outcome cannot be explained by a loss of 14-3-3 binding.

\section{Truncated TASK C-termini are less efficiently phosphorylated in vivo}

14-3-3 binding requires previous phosphorylation of a conserved serine or Threonine residue as part of a 14-3-3 binding motif (exceptions are known that bind 14-3-3 proteins in a phosphorylation independent manner, e.g. R18 and the Arg-based ER retrieval signal of Kir6.2). The C-terminal mode III 14-33 binding motif featured in TASK-1 and TASK-3 matches the recognition site 


\section{Discussion}

of multiple cellular kinases (23), such as cAMP-dependent protein kinase A (PKA), protein kinase C (PKC) and ribosomal S6 kinase (RSK). Phosphorylation assays performed by Mant et al. (2011) demonstrated in vitro that PKA efficiently phosphorylates a conserved serine residue in TASK-1 and TASK-3, which is part of a mode III 14-3-3 binding motif (23). Additionally it was demonstrated that PKA phosphorylates the second serine featured by TASK-1 (S392) efficiently, in vitro. Other kinases tested failed to efficiently phosphorylate either TASK-derived C-terminal peptides, indicating that PKA might also phosphorylate TASK-1 and TASK-3 in vivo (23).

Hoffman et al. (1994) reported, by studying the desensitization of acetylcholine receptors with regard to phosphorylation of different subunits of the protein complex, that by introducing single point mutations, the consensus site of a cellular kinase can be altered allowing phosphorylation of a second residue in a mutation-dependent manner (124). A subsequent study by Shabb et al. (2001) elucidated the effect of mutation or truncation on phosphorylation efficiencies in more detail comparing known PKA consensus sites present in various physiological substrates of PKA. For example, the most frequently and efficiently phosphorylated PKA consensus site found is Arg-Arg-X-Ser, which is highly abundant representing more than half of all PKA recognition sites known (125). The PKA consensus site in TASK-1 and TASK-3 perfectly matches this sequence. In comparison, proteins containing an Arg-X-Ser consensus sequence might also serve as a substrate for PKA, although these recognition sites are phosphorylated with less than $10 \%$ efficiency (125). The efficiency of phosphorylation of different PKA substrates depends on residues flanking the potential phosphoserine or Threonine residue, as well as the accessibility of the residue in a cellular context. According to Shabb et al. (2001) a C-terminal serine residue will be less efficiently phosphorylated (TASK-1 $\Delta$ V394 and TASK-3 $\Delta$ V374)(125).

I determined the phosphorylation state of each reporter protein to further explain observed differences in cell surface expression upon mutation or 
truncation of TASK-1 and TASK-3 C-termini. I found that the TASK-3 Cterminus was efficiently phosphorylated (Figure 16, Lane 1), which is consistent with my observations that the TASK-3 WT reporter protein was mainly localized at the plasma membrane, confirming results reported by Renigunta et al. (2006) that TASK-3 is mainly localizes to the plasma membrane $(11,66)$. Since cell surface expression of TASK-3 was shown to depend on the ability to recruit 14-3-3 proteins $(1,3,11,17,18,64)$, I reason that the phosphorylated TASK-3 C-terminus binds 14-3-3 proteins with high affinity in vivo and is therefore efficiently transported forward to the cell surface. Mutation of S373, which is part of a mode III 14-3-3 binding motif, to alanine abolished interactions with 14-3-3 proteins. Consistent with these findings I observed that the 14-3-3 binding deficient reporter protein, CD8CDP-TASK-3 S373A, failed to reach the surface efficiently and comparable to the truncated reporter protein accumulated in intracellular compartments (64). These observations confirmed previous results reported by O'Kelly et al. (2002), Rajan et al. (2002) and Zuzarte et al. (2009) that efficient transport to the cell surface requires $14-3-3$ binding $(11,17,18)$. Reporter proteins, in which the non-canonical ER retention and retrieval motif, present at the distal C-terminus (K369A), was mutated, displayed a similar cell surface expression compared to the TASK-3 WT reporter-protein. The reporter protein was as efficiently phosphorylated as the TASK-3 wild type reporter protein and therefore I expected the reporter protein to bind 14-3-3 proteins with high affinity. Major differences in phosphorylation were observed for the truncated TASK-3 reporter protein. Phostag SDS-PAGE revealed that approximately $50 \%$ of the truncated reporter-protein was phosphorylated in vivo. These observations suggest that either the recognition of the truncated $\mathrm{C}$-terminus by PKA was affected and consequently the truncated C-terminus was less efficiently phosphorylated, or that 14-3-3 binding to the C-terminus only insufficiently protected the truncated C-terminus from dephosphorylation, allowing access to cellular phosphatases. I explored the first possibility further and found that the truncated TASK-3 C-terminus was less efficiently phosphorylated by PKA than the TASK-3 WT C-terminus, in vitro, 


\section{Discussion}

demonstrating that truncation of the TASK-3 C-terminus affects the recognition by PKA as well as 14-3-3 binding. I conclude that the lack of cell surface expression of the truncated reporter protein of TASK-3 can be explained by cooperative effects of less efficient phosphorylation of the client protein and more frequent dephosphorylation of the reporter protein due to the reduced efficacy of 14-3-3 binding.

Mant et al. (2011) reported that both serine residues present in TASK-1 are efficiently phosphorylated by PKA, in vitro (S392, S393)(23). I demonstrated that phosphorylation of the second serine residue present in TASK-1 (S392) has a potential inhibitory effect on 14-3-3 binding and therefore affects the forward transport of the reporter protein to the cell surface. Furthermore, I investigated the phosphorylation state of different TASK-1-derived reporter proteins in vivo and if S392 could be phosphorylated in a cellular context. Interestingly, the TASK-1 wild-type reporter protein was efficiently phosphorylated, but solely one protein band corresponding to the single phosphorylated protein was observed. Single serine to alanine mutants illustrate that S392 was only weakly phosphorylated in vivo in absence of S393. On the other hand S393 was efficiently phosphorylated, in absence of S392. Comparable to observations made for the TASK-3 K369A reporter protein, the analogous TASK-1 K389A reporter protein was phosphorylated efficiently and displayed a protein band corresponding to the single phosphorylated protein. I attribute these observations to differential 14-3-3 binding affinities for different TASK-1 derived reporter proteins. In this model 14-3-3 binding is thought to protect the phosphorylated side-chain of TASK-1 from dephosphorylation by cellular phosphatases (117). Since I have previously shown that phosphorylation of S392 substantially decreased 14-3-3 binding affinities to TASK-1 derived peptides (in vitro), reporter proteins in which S392 is accessible to cellular kinases should bind 14-3-3 proteins less efficiently and S392 should consequently be more prone to dephosphorylation by cellular phosphatases. 


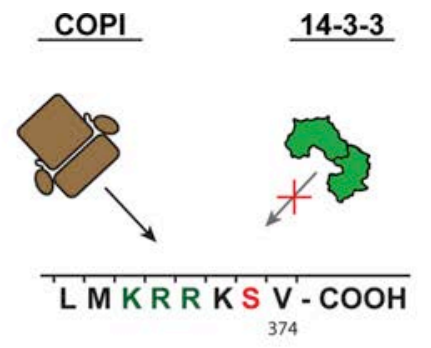

\section{Basal}

Regulated
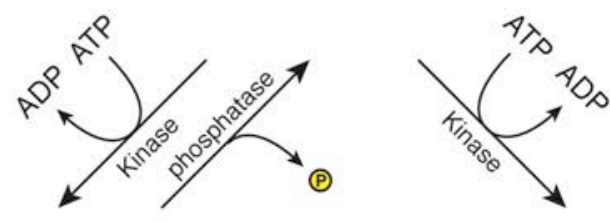

A

phosphatase

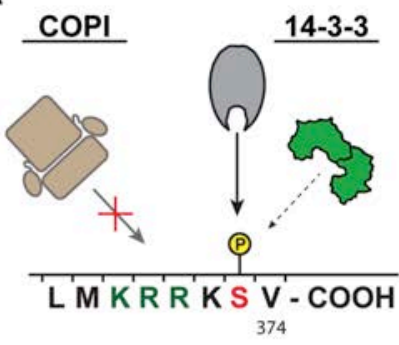

B

phosphatase
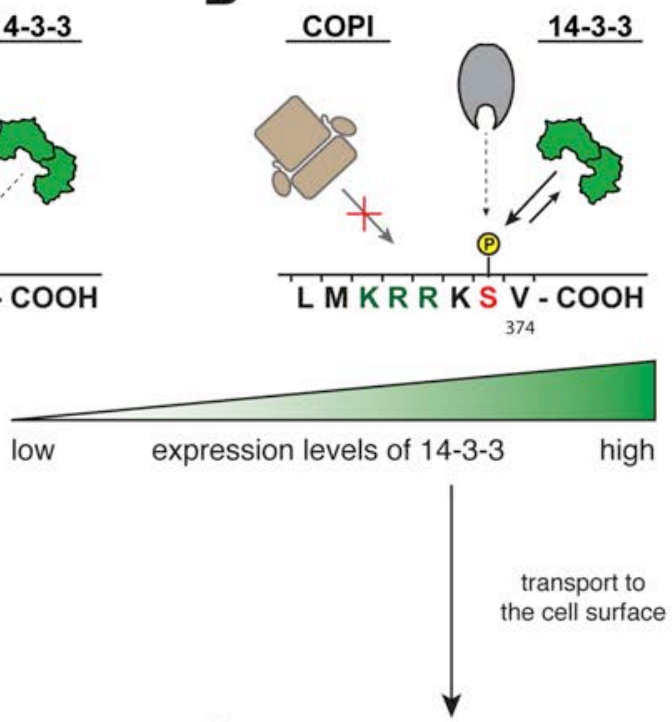

C

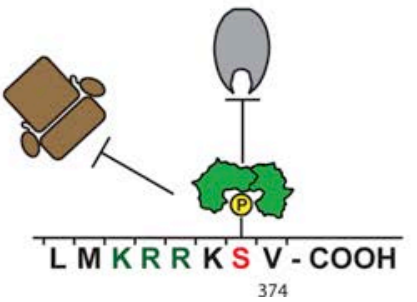

Figure 28: Proposed mechanism that modulates the cell surface expression of TASK-3. Basal state: Interactions with the COPI vesicle coat mediate the retention of the protein in the early compartments of the secretory pathway. A: Phosphorylation of S373 by cellular kinases, potentially PKA (23), leads to the recruitment of 14-3-3 proteins. Low 14-3-3 expression levels permit the rapid association of 14-3-3 and the phosphorylated TASK-3 C-terminus. The absence of 14-3-3 proteins allows for a more frequent access of cellular phosphatase to this trafficking control region. Dephosphorylation of $\mathrm{S} 373$ converts the channel into its basal state. B: After 


\section{Discussion}

phosphorylation of the conserved serine residue (S373), which is part of a mode III 14-3-3 binding motif $(14,15), 14-3-3$ proteins bind the C-terminus of TASK-3 with high affinity and enable the forward transport of the channel to the cell surface. Low and moderate expression levels will enable immediate masking of the phosphoserine residue, because of the high affinity of 14-3-3 proteins for the phosphorylated TASK3 C-terminus, thereby limiting the access of cellular phosphatases to the traffickingcontrol region. The binding equilibrium between association (big arrow) and dissociation (small arrow) of 14-3-3 and the phosphorylated TASK-3 C-terminus is indicated. C: Association with 14-3-3 facilitates the forward transport of the channel to cell surface.

CD8-CFP-TASK-1 S392A was phosphorylated efficiently, hence these reporter proteins should bind 14-3-3 proteins with higher affinity than reporter proteins in which both serine residues, or only S392 are accessible to cellular kinases and the phosphorylated side chains (pS393) should therefore be efficiently protected from dephosphorylation by cellular phosphatases. Deletion of the distal valine residue also resulted in a decrease of phosphorylation efficiency of the TASK-1 C-terminus, indicating similar changes in recognition of the phosphorylation consensus site by PKA (125). In vitro data suggests that 14-3-3 binding is lost upon truncation of the TASK1 C-terminus, again, highlighting differences between TASK-1 and TASK-3 Ctermini and further emphasizing the role of S392 in TASK-1 protein transport (64). My observations demonstrate that the cell surface expression of TASK-1 can be modulated by other factors, such as phosphorylation of S392 and might thereby render TASK-1 more amenable to regulation. In this study I utilized the last 15 amino acids of either TASK-1 or TASK-3 to simplify the interpretation of our observations, yet I observed distinct effects imposed by minor variations in amino acid sequence. To further appreciate the contribution of other accessory proteins, such as p11 $(19,66,113)$, it is necessary to perform experiments with full length TASK-1 and TASK-3 Ctermini. 
In summary, my observations suggest a mechanism, by which the mutually exclusive binding of 14-3-3 and COPI to the trafficking control regions present in TASK-1 and TASK-3 is regulated by signal transduction events, such as phosphorylation of the client protein by kinases (e.g. PKA), dephosphorylation of the client protein by cellular phosphatases and the expression levels of 143-3 proteins in different cell-types and tissues. Furthermore our findings demonstrate that binding events are not competitive as previously assumed $(11,17,18)$. The substantially lower affinity of 14-3-3 for TASK-1 WT pS393, compared to TASK-3 WT pS373, and COPI for TASK-1 WT (CT15) allows for a dynamic steady state in which kinases and phosphatases can access and modify the trafficking control region more frequently, than in the case of TASK-3. Phosphorylation of S392 will reduce the efficacy by which 14-3-3 can release the channel from COPI-mediated ER retention, allowing for dephosphorylation by cellular phosphatases. On the other hand phosphorylation of S393 will allow for moderate affinity binding of 14-3-3, protecting the phosphorylated amino acid side chain from dephosphorylation, and therefore facilitating anterograde transport of the channel to the cell surface. For TASK-3 the access of cellular kinases to the trafficking control region present at the distal C-terminus is limited. Upon phosphorylation TASK3 binds 14-3-3 proteins with high affinity allowing for release of the channel from ER retention. The unphosphorylated $\mathrm{C}$-terminus contains a high affinity COPI binding site and should therefore be retained efficiently. This is exactly what I observed for the 14-3-3 binding deficient reporter protein of TASK-3 (CD8-CFP-TASK-3 S373A). Replacing K369 with alanine did not further increase the amount of reporter protein expressed at the cell surface. Either the reporter protein was sufficiently over expressed and the density of reporter protein at the cell surface reached saturation, or the ER retention activity of the TASK-3 K369A reporter protein was sustained by a second dibasic and canonical ER retention and retrieval motif presented by TASK-3 (RKXX, (8)). For comparison the last six amino acid residues presented by either TASK-3 WT, K ${ }^{369}$ RRKSV-COOH, or TASK-3 K369A, A ${ }^{369}$ RRKSV-COOH. 


\section{Discussion}
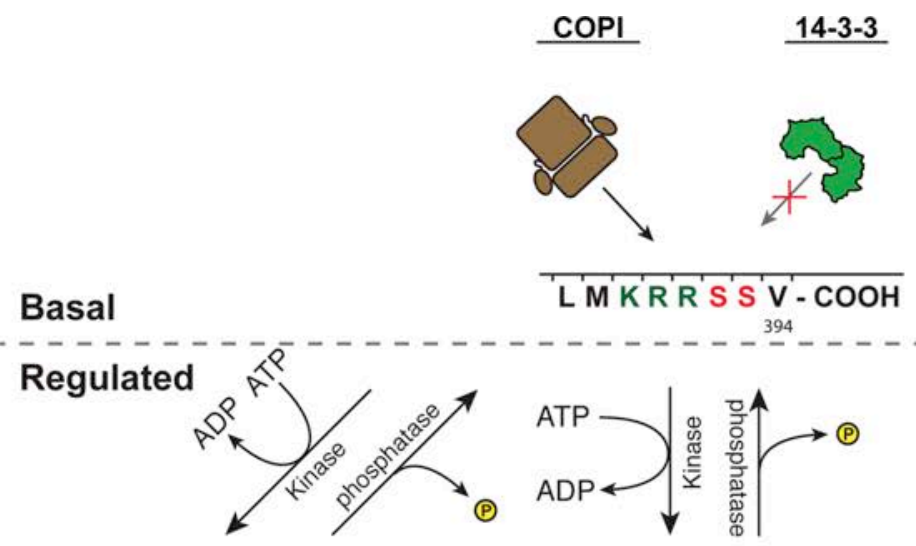

low expression levels of 14-3-3

high

A

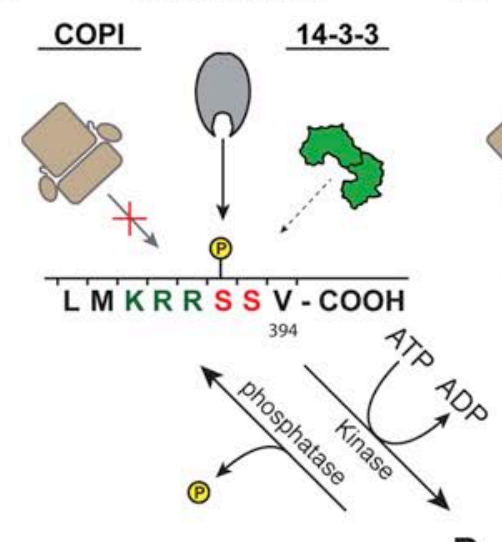

B phosphatase
C phosphatase

C

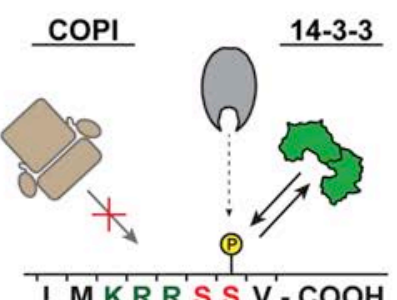

'L'M K'R'R'S' V'- $\mathrm{COOH}$

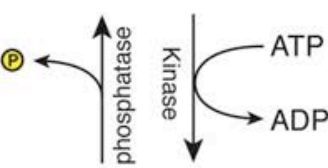
phosphatase

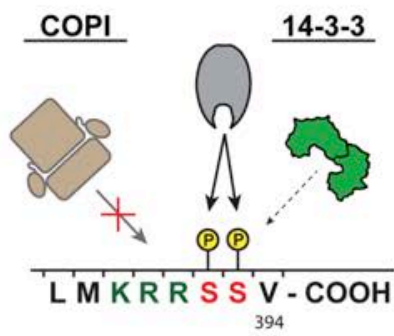

E

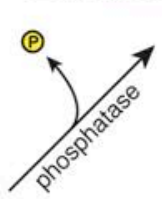
394
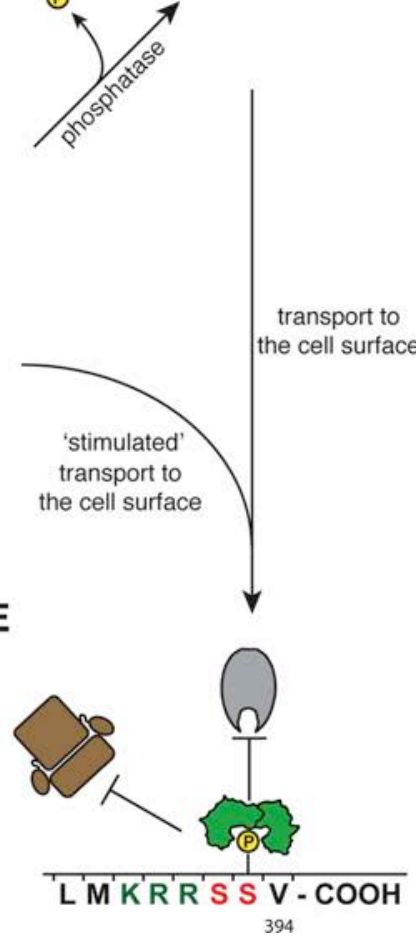

Figure 29: Proposed mechanism that modulates the cell surface expression of TASK-1. Basal state: Interactions with the COPI vesicle coat mediate the retention the protein in the early compartments of the secretory pathway. The protein shuttles between ER and Golgi. A: After the phosphorylation of S392 by cellular kinases, potentially PKA (23), because of the low binding affinities of different 14-3-3 isoforms, 
14-3-3 binding does not efficiently protect the trafficking control region present at the distal C-terminus of TASK-1 from dephosphorylation, facilitating the access of cellular phosphatase to this trafficking control region. Dephosphorylation of S392 converts the channel into its basal state. B: After phosphorylation of the conserved serine residue (S393), which is part of a mode III 14-3-3 binding motif $(14,15), 14-3-3$ proteins can bind the C-terminus of TASK-1 with moderate affinity. Low expression levels facilitate the more frequent access of cellular phosphatases to this trafficking control. C: Dephosphorylation converts the channel into its basal state. C: High expression levels of 14-3-3 allow for a rapid masking of the phosphoserine residue, thereby limiting the access of cellular phosphatases to the trafficking-control region present in TASK-1, enabling the forward transport of the channel to the cell surface. The significantly lower binding affinity of 14-3-3 proteins for the phosphorylated TASK-1 C-terminus might lead to a more dynamic equilibrium between association (upper arrow) and dissociation of 14-3-3 proteins (lower arrow), thereby facilitating the access of cellular phosphatases to the trafficking control region present in TASK1. Compared to TASK-3, high expression levels of 14-3-3 might be insufficient to saturate available binding sites present TASK-1 pS393, thus this trafficking control region is less efficiently protected from dephosphorylation. D: A double phosphorylation of the TASK-1 C-terminus can be achieved by phosphorylation of $\mathbf{A}$ and B. Stimulated activity of PKA might facilitate the double phosphorylation of the channel in vivo, thereby uncoupling the cell surface transport of TASK-1 from the antagonistic actions of COPI and 14-3-3. Another possible scenario: The low binding affinities of 14-3-3 do not efficiently protect the channel from dephosphorylation, facilitating the frequent access of cellular phosphatases to the doubly phosphorylated trafficking control region. Dephosphorylation might potentially lead to A, B or C. E: Once associated with 14-3-3, the TASK-1 channel is transported to the cell surface. 
Discussion 


\section{Appendix}

\section{Abbreviations}

\begin{tabular}{|c|c|}
\hline$A$ & ampere \\
\hline ATP & adenosine tri-phosphate \\
\hline CFP & cyan fluorescent protein \\
\hline DNA & deoxyribonucleic acid \\
\hline NTPs & deoxyribonuclotide triphosphates \\
\hline EM & energy mix \\
\hline ER & endoplasmic reticulum \\
\hline FACS & fluorescence-activated cell sorting \\
\hline FP & fluorescence polarization \\
\hline$g$ & gram \\
\hline GST & glutathione-S-transferase \\
\hline $\mathrm{h}$ & hour \\
\hline $\mathrm{kb}$ & kilo-base pair \\
\hline $\mathrm{kDa}$ & kilo-Dalton \\
\hline I & liter \\
\hline LB & Luria-Bertani \\
\hline$\lambda P P($ ase $)$ & lambda protein phosphatase \\
\hline $\mathrm{M}$ & molar \\
\hline $\min$ & minute \\
\hline MBP & maltose binding protein \\
\hline n.b. & no binding \\
\hline Pol & polarization units \\
\hline$\mu$ & micro \\
\hline$\mu \mathrm{RIU}$ & micro refractive index units \\
\hline $\mathrm{nm}$ & nanometer \\
\hline ON & overnight \\
\hline PAGE & polyacrylamide gel electrophoresis \\
\hline PBS & phosphate-buffered saline \\
\hline PCR & polymerase chain reaction \\
\hline PKA & protein kinase $\mathrm{A}$ \\
\hline
\end{tabular}




\section{Appendix}

$\begin{array}{ll}\text { rpm } & \text { revolution per minute } \\ \text { S } & \text { second } \\ \text { SDS } & \text { sodium dodecyl sulfate } \\ \text { s.e.m. } & \text { standard error of the mean } \\ \text { SPR } & \text { surface plasmon resonance } \\ \text { U } & \text { unit } \\ \text { V } & \text { volt } \\ \text { v/v } & \text { volume per volume } \\ \text { w/v } & \text { weight per volume } \\ \text { w/w } & \text { weight per weight } \\ \text { WT } & \text { wild type }\end{array}$

\section{Buffers used in this thesis:}

Binding buffer:

GST-breaking buffer:

50 mM Tris- $\mathrm{HCl}, \mathrm{pH} 7.5$

20 mM HEPES, pH 6.5

$150 \mathrm{mM} \mathrm{NaCl}$

$150 \mathrm{mM}$ KOAc

$10 \mathrm{mM} \mathrm{MgCl} 2$

$5 \mathrm{mM} \mathrm{Mg}(\mathrm{OAc}) 2$

1 mM EDTA

Blocking buffer:

$1 \mathrm{mM}$ DTT

25 mM Tris- $\mathrm{HCl}, \mathrm{pH} 7.4$

$1 \mathrm{mM}$ PMSF

$135 \mathrm{mM} \mathrm{NaCl}$

$3 \mathrm{mM} \mathrm{KCl}$

GST-elution buffer:

$5 \%$ milk powder $(\mathrm{w} / \mathrm{v})$

20 mM HEPES, pH 8.5

$0.02 \%$ NP-40

$150 \mathrm{mM}$ KOAc

$5 \mathrm{mM} \mathrm{Mg}(\mathrm{OAc}) 2$

FP buffer:

1 mM EDTA

20 mM MOPS, pH 7.4

$1 \mathrm{mM}$ DTT

$150 \mathrm{mM} \mathrm{NaCl}$

$1 \mathrm{mM}$ PMSF

$0.005 \%$ CHAPS

$20 \mathrm{mM}$ glutathione 
MBP-column buffer:

50 mM Tris- $\mathrm{HCl}, \mathrm{pH} 7.5$

$150 \mathrm{mM} \mathrm{NaCl}$

$5 \mathrm{mM} \mathrm{MgCl} 2$

$1 \mathrm{mM}$ PMSF

MBP-elution buffer:

50 mM Tris- $\mathrm{HCl}, \mathrm{pH} 7.5$

$150 \mathrm{mM} \mathrm{NaCl}$

$5 \mathrm{mM} \mathrm{MgCl} 2$

$1 \mathrm{mM}$ PMSF

20 mM D-Maltose

Membrane preparation buffer:

20 mM HEPES, pH 7.4

$50 \mathrm{mM} \mathrm{NaCl}$

$0.32 \mathrm{M}$ sucrose

2 mM EDTA

add cOmplete EDTA-free protease

inhibitor cocktail

add $50 \mu \mathrm{M}$ PKA inhibitor $\mathrm{H}-89$

Phosphoryltion buffer:

20 mM HEPES, pH 6.8

$150 \mathrm{mM}$ KOAc

$5 \mathrm{mM} \mathrm{Mg}(\mathrm{OAc}) 2$

1 mM EDTA

$1 \mathrm{mM}$ DTT

$2 \%$ glycerol
SDS-running buffer:

25 mM Tris- $\mathrm{HCl}$, pH 8.3

250 mM Glycine

$0.1 \%$ SDS

Solubilization buffer:

50 mM Tris-HCl, $\mathrm{pH} 7.5$

$100 \mathrm{mM} \mathrm{NaCl}$

5 mM EDTA

2.5 mM EGTA

1.5\% Triton $\mathrm{X}-100$

$0.75 \%$ Na-deoxycholate

$0.1 \%$ SDS

SPR-running buffer:

20 mM HEPES, pH 7.4

$150 \mathrm{mM} \mathrm{NaCl}$

0.005\% TWEEN-20

Transfer buffer:

25 mM Tris, pH 8.3

192 mM Glycine 


\section{Appendix}

Media used in this thesis:

LB (Luria-Bertani) medium:

$10 \mathrm{~g} / \mathrm{l} \mathrm{Bacto}$ tryptone

$5 \mathrm{~g} / \mathrm{l}$ Bacto yeast extract

$10 \mathrm{~g} / \mathrm{l} \mathrm{NaCl}$

$\mathrm{pH} 7.0$

SOC medium:

$20 \mathrm{~g} / \mathrm{l} \mathrm{Bacto}$ tryptone

$5 \mathrm{~g} / \mathrm{l}$ Bacto yeast extract

$0.5 \mathrm{~g} / \mathrm{l} \mathrm{NaCl}$

$2.5 \mathrm{mM} \mathrm{KCl}$

$10 \mathrm{mM} \mathrm{MgCl} 2$

20 mM Glucose

$\mathrm{pH} 7.4$

2YT medium:

$16 \mathrm{~g} / \mathrm{l}$ Bacto tryptone

$10 \mathrm{~g} / \mathrm{l}$ Bacto yeast extract

$5 \mathrm{~g} / \mathrm{NaCl}$

30 mM K2HPO4

$2 \%$ Glycerol $(v / v)$

$\mathrm{pH} 7.0$ 


\section{Chemical list}

\begin{tabular}{|c|c|c|}
\hline Chemical & Abbreviation & Provider \\
\hline Acetic acid & $\mathrm{CH} 3 \mathrm{COOH}$ & AppliChem \\
\hline Acrylamide & & AppliChem \\
\hline Ammonium persulfate & APS & Biomol \\
\hline & & Feinchemiekalien \\
\hline & & $\mathrm{GmbH}$ \\
\hline $\begin{array}{l}\text { 3-[(3-Cholamidopropyl)- } \\
\text { dimethylammonio]-1-propane- } \\
\text { sulfonate }\end{array}$ & CHAPS & Sigma \\
\hline Coomassie brilliant blue & & AppliChem \\
\hline D-Maltose & & AppliChem \\
\hline Dimethyl sulfoxide & DMSO & AppliChem \\
\hline Dithiothreitol & DTT & AppliChem \\
\hline $\begin{array}{l}N \text {-(3-Dimethylaminopropyl)- } N^{\prime} \text { - } \\
\text { ethylcarbodiimide hydrochloride }\end{array}$ & EDC & Sigma \\
\hline Ethylenediaminetetraacetic acid & EDTA & AppliChem \\
\hline $\begin{array}{l}\text { Ethylene glycol-bis-(2-amino- } \\
\text { ethylether)- } N, N, N^{\prime}, N^{\prime} \text {-tetraacetic acid }\end{array}$ & EGTA & AppliChem \\
\hline Ethanol & $\mathrm{EtOH}$ & Carl Roth \\
\hline Ethanolamine & & Xantex bioanalytics \\
\hline Glucose & & Carl Roth \\
\hline Glutathione & GSH & GE Healthcare \\
\hline Glycine & Gly & Carl Roth \\
\hline Hydrochloric acid & $\mathrm{HCl}$ & AppliChem \\
\hline $\begin{array}{l}\text { 2-[4-(2-hydroxyethyl)piperazin-1- } \\
\text { yl]ethanesulfonic acid }\end{array}$ & HEPES & AppliChem \\
\hline Isopropyl- $\beta$-D-thiogalactopyranosid & IPTG & AppliChem \\
\hline Potassium chloride & $\mathrm{KCl}$ & Merck \\
\hline Potassium acetate & KOAC & AppliChem \\
\hline Magnesium acetate & $\mathrm{Mg}(\mathrm{OAc})_{2}$ & Merck \\
\hline Magnesium chloride & $\mathrm{MgCl}_{2}$ & Carl Roth \\
\hline Manganese(II) chloride & $\mathrm{MnCl}_{2}$ & AppliChem \\
\hline
\end{tabular}


Appendix

\begin{tabular}{lll} 
Chemical & Abbreviation & Provider \\
\hline $\begin{array}{l}\text { 3-Morpholinopropane-1-sulfonic acid } \\
\text { Na-deoxycholate }\end{array}$ & MOPS & Carl Roth \\
$\begin{array}{l}\text { Disodium hydrogen phosphate } \\
\text { dihydrate }\end{array}$ & $\mathrm{Na}_{2} \mathrm{HPO}_{4} \times 2 \mathrm{H}_{2} \mathrm{O}$ & Sigma \\
Sodium chloride & & AppliChem \\
Sodium acetate & $\mathrm{NaCl}$ & Carl Roth \\
Sodium hydroxide & $\mathrm{NaOAc}$ & Carl Roth \\
N-hydroxysuccinimide & $\mathrm{NaOH}$ & Carl Roth \\
NP-40 & $\mathrm{NHS}$ & Xantex bioanalytics \\
Phos-tag acrylamide & & AppliChem \\
& $\mathrm{Phos}$-tag & Waka Chemicals \\
Phenylmethanesulfonyl fluoride & & GmbH \\
Trichloracetic acid & $\mathrm{PMSF}$ & Roche \\
2-Amino-2-hydroxymethyl-propane- & TCA & AppliChem \\
1,3-diol & & AppliChem \\
TWEEN-20 & & Carl Roth \\
Sucrose & & Fisher Scientific \\
dNTP set & & Thermo Scientific
\end{tabular}




\section{References}

1. A. J. Smith, J. Daut, B. Schwappach, Membrane proteins as 14-3-3 clients in functional regulation and intracellular transport. Physiology (Bethesda). 26, 181-191 (2011).

2. N. Decher, A. K. Kiper, C. Rolfes, E. Schulze-Bahr, S. Rinné, The role of acid-sensitive two-pore domain potassium channels in cardiac electrophysiology: focus on arrhythmias. Pflugers Arch. 467, 1055-67 (2015).

3. M. Kilisch, O. Lytovchenko, B. Schwappach, V. Renigunta, J. Daut, The role of protein-protein interactions in the intracellular traffic of the potassium channels TASK-1 and TASK-3. Pflügers Arch. - Eur. J. Physiol. 467, 1105-1120 (2015).

4. J. Martin, F. Ulrich Hartl, Chaperone-assisted protein folding. Curr. Opin. Struct. Biol. 7, 41-52 (1997).

5. F. U. Hartl, Chaperone-assisted protein folding: the path to discovery from a personal perspective. Nat. Med. 17, 1206-1210 (2011).

6. E. C. Arakel et al., Tuning the electrical properties of the heart by differential trafficking of KATP ion channel complexes. J. Cell Sci. 127, 2106-19 (2014).

7. D. M. Hutt, W. E. Balch, Expanding proteostasis by membrane trafficking networks. Cold Spring Harb. Perspect. Med. 3, 1-21 (2013).

8. S. H. Keller, J. Lindstrom, M. Ellisman, P. Taylor, Adjacent Basic Amino Acid Residues Recognized by the COP I Complex and Ubiquitination Govern Endoplasmic Reticulum to Cell Surface Trafficking of the Nicotinic Acetylcholine Receptor??-Subunit. J. Biol. Chem. 276, 1838418391 (2001).

9. A. Eugster, G. Frigerio, M. Dale, R. Duden, The alpha- and beta'-COP WD40 domains mediate cargo-selective interactions with distinct dilysine motifs. Mol. Biol. Cell. 15, 1011-23 (2004).

10. K. Michelsen et al., Novel cargo-binding site in the $\beta$ and $\delta$ subunits of coatomer. J. Cell Biol. 179, 209-217 (2007). 


\section{References}

11. M. Zuzarte et al., Intracellular traffic of the K+ channels TASK-1 and TASK-3: role of $\mathrm{N}$ - and $\mathrm{C}$-terminal sorting signals and interaction with 14-3-3 proteins. J. Physiol. 587, 929-52 (2009).

12. J. S. Bonifacino, J. Lippincott-Schwartz, Coat proteins: shaping membrane transport. Nat. Rev. Mol. Cell Biol. 4, 409-414 (2003).

13. M. B. Yaffe et al., The structural basis for 14-3-3:phosphopeptide binding specificity. Cell. 91, 961-971 (1997).

14. S. Ganguly et al., Melatonin synthesis: 14-3-3-dependent activation and inhibition of arylalkylamine $\mathrm{N}$-acetyltransferase mediated by phosphoserine-205. Proc. Natl. Acad. Sci. U. S. A. 102, 1222-1227 (2005).

15. B. Coblitz et al., C-terminal recognition by 14-3-3 proteins for surface expression of membrane receptors. J. Biol. Chem. 280, 36263-36272 (2005).

16. K. Michelsen et al., A multimeric membrane protein reveals 14-3-3 isoform specificity in forward transport in yeast. Traffic. 7, 903-916 (2006).

17. S. Rajan et al., Interaction with 14-3-3 proteins promotes functional expression of the potassium channels TASK-1 and TASK-3. J. Physiol. 545, 13-26 (2002).

18. I. O’Kelly, M. H. Butler, N. Zilberberg, S. a N. Goldstein, Forward transport: 14-3-3 Binding overcomes retention in endoplasmic reticulum by dibasic signals. Cell. 111, 577-588 (2002).

19. I. O'Kelly, S. A. N. Goldstein, Forward Transport of K2p3.1: mediation by 14-3-3 and COPI, modulation by p11. Traffic. 9, 72-8 (2008).

20. L. Lum, M. S. Reid, C. P. Blobel, Intracellular maturation of the mouse metalloprotease disintegrin MDC15. J. Biol. Chem. 273, 26236-26247 (1998).

21. N. J. Gödde, G. M. D’Abaco, L. Paradiso, U. Novak, Efficient ADAM22 surface expression is mediated by phosphorylation-dependent interaction with 14-3-3 protein family members. J. Cell Sci. 119, 3296305 (2006). 
22. O. P. Hamill, A. Marty, E. Neher, B. Sakmann, F. J. Sigworth, Improved patch-clamp techniques for high-resolution current recording from cells and cell-free membrane patches. Pflügers Arch. Eur. J. Physiol. 391, 85100 (1981).

23. A. Mant, D. Elliott, P. a. Eyers, I. M. O'Kelly, Protein kinase A is central for forward transport of two-pore domain potassium channels K2P3.1 and K2P9.1. J. Biol. Chem. 286, 14110-14119 (2011).

24. S. Feliciangeli, F. C. Chatelain, D. Bichet, F. Lesage, The family of $K_{2 P}$ channels: salient structural and functional properties. J. Physiol. 593, 2587-2603 (2015).

25. V. Renigunta, G. Schlichth??rl, J. Daut, Much more than a leak: structure and function of K2P-channels. Pflugers Arch. Eur. J. Physiol., 867-894 (2015).

26. A. D. O'Connell, M. J. Morton, M. Hunter, Two-pore domain K+ channels-molecular sensors. Biochim. Biophys. Acta. 1566, 152-161 (2002).

27. I. D. Kerr, M. S. P. Sansom, Cation selectivity in ion channels. Nature. 373 (1995), p. 112.

28. L. Heginbotham, Z. Lu, T. Abramson, R. MacKinnon, Mutations in the K+ channel signature sequence. Biophys. J. 66, 1061-7 (1994).

29. A. Miller, S. Long, Crystal Structure of the Human Two - Pore Domain Potassium Channel K2P1. Science (80-. ). 335, 432 (2012).

30. S. G. Brohawn, E. B. Campbell, R. MacKinnon, Domain-swapped chain connectivity and gated membrane access in a Fab-mediated crystal of the human TRAAK K+ channel. Proc. Natl. Acad. Sci. U. S. A. 110, 2129-34 (2013).

31. F. Lesage et al., TWIK-1, a ubiquitous human weakly inward rectifying $\mathrm{K}+$ channel with a novel structure. EMBO J. 15, 1004-11 (1996).

32. R. A. Chavez et al., TWIK-2, a new weak inward rectifying member of the tandem pore domain potassium channel family. J. Biol. Chem. 274, 7887-7892 (1999).

33. M. Salinas et al., Cloning of a new mouse two-P domain channel 


\section{References}

subunit and a human homologue with a unique pore structure. J. Biol. Chem. 274, 11751-11760 (1999).

34. M. Fink et al., Cloning, functional expression and brain localization of a novel unconventional outward rectifier $\mathrm{K}+$ channel. EMBO J. 15, 68546862 (1996).

35. H. Bang, Y. Kim, D. Kim, TREK-2, a new member of the mechanosensitive tandem pore $\mathrm{K}+$ channel family. J. Biol. Chem. 275, 17412-17419 (2000).

36. M. Fink et al., A neuronal two $\mathrm{P}$ domain $\mathrm{K}+$ channel activated by arachidonic acid and polyunsaturated fatty acid. Embo J.17, 32973308 (1998).

37. F. Duprat et al., TASK, a human background $\mathrm{K}+$ channel to sense external pH variations near physiological pH. EMBO J. 16, 5464-5471 (1997).

38. Y. Kim, H. Bang, D. Kim, TASK-3, a new member of the tandem pore K+ channel family. J. Biol. Chem. 275, 9340-9347 (2000).

39. S. Rajan et al., TASK-3, a novel tandem pore domain acid-sensitive K+ channel. An extracellular histidine as pH sensor. J. Biol. Chem. 275, 16650-16657 (2000).

40. I. Ashmole, P. A. Goodwin, P. R. Stanfield, TASK-5, a novel member of the tandem pore K+ channel family. Pflugers Arch. Eur. J. Physiol. 442, 828-833 (2001).

41. C. Karschin et al., Expression pattern in brain of TASK-1, TASK-3, and a tandem pore domain $\mathrm{K}(+)$ channel subunit, TASK-5, associated with the central auditory nervous system. Mol. Cell. Neurosci. 18, 632-48 (2001).

42. D. Kim, C. Gnatenco, TASK-5, a new member of the tandem-pore K(+) channel family. Biochem. Biophys. Res. Commun. 284, 923-930 (2001).

43. R. Reyes et al., Cloning and expression of a novel $\mathrm{pH}$-sensitive two pore domain potassium channel from human kidney. J. Biol. Chem. 273, 30863-30869 (1998). 
44. C. Girard et al., Genomic and functional characteristics of novel human pancreatic $2 \mathrm{P}$ domain $\mathrm{K}(+)$ channels. Biochem. Biophys. Res. Commun. 282, 249-256 (2001).

45. N. Decher et al., Characterization of TASK-4, a novel member of the $\mathrm{pH}-$ sensitive, two-pore domain potassium channel family. FEBS Lett. 492, 84-89 (2001).

46. S. Rajan et al., THIK-1 and THIK-2, a Novel Subfamily of Tandem Pore Domain K+ Channels. J. Biol. Chem. 276, 7302-7311 (2001).

47. Y. Sano et al., A novel two-pore domain $\mathrm{K}+$ channel, TRESK, is localized in the spinal cord. J. Biol. Chem. 278, 27406-27412 (2003).

48. G. Czirják, P. Enyedi, Formation of functional heterodimers between the TASK-1 and TASK-3 two-pore domain potassium channel subunits. J. Biol. Chem. 277, 5426-5432 (2002).

49. A. P. Berg, E. M. Talley, J. P. Manger, D. a Bayliss, Motoneurons express heteromeric TWIK-related acid-sensitive K+ (TASK) channels containing TASK-1 (KCNK3) and TASK-3 (KCNK9) subunits. J. Neurosci. 24, 6693-6702 (2004).

50. D. Kang, J. Han, E. M. Talley, D. A. Bayliss, D. Kim, Functional expression of TASK-1/TASK-3 heteromers in cerebellar granule cells. J. Physiol. 554, 64-77 (2004).

51. P. M. Larkman, E. M. Perkins, A TASK-like $\mathrm{pH}$ - and amine-sensitive "leak" $\mathrm{K}+$ conductance regulates neonatal rat facial motoneuron excitability in vitro. Eur. J. Neurosci. 21, 679-91 (2005).

52. D. Kim, E. J. Cavanaugh, I. Kim, J. L. Carroll, Heteromeric TASK$1 /$ TASK-3 is the major oxygen-sensitive background $K+$ channel in rat carotid body glomus cells. J. Physiol. 587, 2963-75 (2009).

53. P. Enyedi, G. Czirják, I. Introduction, Molecular background of leak K+ currents: two-pore domain potassium channels. Physiol. Rev. 90, 559605 (2010).

54. S. Blin et al., Tandem pore domain halothane-inhibited $\mathrm{K}+$ channel subunits THIK1 and THIK2 assemble and form active channels. J. Biol. Chem. 289, 28202-28212 (2014). 


\section{References}

55. L. D. Plant, L. Zuniga, D. Araki, J. D. Marks, S. a N. Goldstein, SUMOylation silences heterodimeric TASK potassium channels containing K2P1 subunits in cerebellar granule neurons. Sci. Signal. 5, ra84 (2012).

56. E. Mi Hwang et al., A disulphide-linked heterodimer of TWIK-1 and TREK-1 mediates passive conductance in astrocytes. Nat. Commun. 5, 3227 (2014).

57. P. Enyedi, G. Czirják, Properties, regulation, pharmacology, and functions of the K2P channel, TRESK. Pflügers Arch. - Eur. J. Physiol. (2014), doi:10.1007/s00424-014-1634-8.

58. I. O'Kelly, Endocytosis as a mode to regulate functional expression of two-pore domain potassium (K2P) channels. Pflügers Arch. - Eur. J. Physiol. 467, 1133-1142 (2015).

59. D. Mu et al., Genomic amplification and oncogenic properties of the KCNK9 potassium channel gene. Cancer Cell. 3, 297-302 (2003).

60. L. Pei et al., Oncogenic potential of TASK3 (Kcnk9) depends on K+ channel function. Proc. Natl. Acad. Sci. U. S. A. 100, 7803-7807 (2003).

61. M. Zanzouri, I. Lauritzen, M. Lazdunski, A. Patel, The background K+ channel TASK-3 is regulated at both the transcriptional and posttranscriptional levels. Biochem. Biophys. Res. Commun. 348, 13501357 (2006).

62. M. Zanzouri et al., Membrane potential-regulated transcription of the resting $\mathrm{K}+$ conductance TASK-3 via the calcineurin pathway. J. Biol. Chem. 281, 28910-28918 (2006).

63. G. Sandoz et al., Mtap2 Is a Constituent of the Protein Network That Regulates Twik-Related K 2 Channel Expression and Trafficking. 28, 8545-8552 (2008).

64. M. Kilisch, O. Lytovchenko, E. C. Arakel, D. Bertinetti, A dual phosphorylation switch controls 14-3-3-dependent cell surface expression of TASK-1, 831-842 (2016).

65. G. Sandoz et al., AKAP150, a switch to convert mechano-, pH- and 
arachidonic acid-sensitive TREK K(+) channels into open leak channels. EMBO J. 25, 5864-5872 (2006).

66. V. Renigunta et al., The retention factor p11 confers an endoplasmic reticulum-localization signal to the potassium channel TASK-1. Traffic. 7, 168-181 (2006).

67. V. Renigunta et al., Cooperative endocytosis of the endosomal SNARE protein syntaxin-8 and the potassium channel TASK-1. Mol. Biol. Cell. 25, 1877-91 (2014).

68. S. H. Barondes, Physiological and Biochemical Aspects of Nervous Integration. A symposium, Woods Hole, Mass., 1967. Francis D. Carlson, Ed. Prentice-Hall, Englewood Cliffs, N. J., 1968. viii + 392 pp., illus. \$7. Science (80-. ). 162, 1378-1378 (1968).

69. P. F. Erickson, B. W. Moore, Investigation of the axonal transport of three acidic, soluble proteins (14-3-2, 14-3-3, and S-100) in the rabbit visual system. J. Neurochem. 35, 232-241 (1980).

70. P. F. Boston, P. Jackson, P. a Kynoch, R. J. Thompson, Purification, properties, and immunohistochemical localisation of human brain 14-3-3 protein. J. Neurochem. 38, 1466-1474 (1982).

71. P. F. Boston, P. Jackson, R. J. Thompson, Human 14-3-3 protein: radioimmunoassay, tissue distribution, and cerebrospinal fluid levels in patients with neurological disorders. J. Neurochem. 38, 1475-1482 (1982).

72. D. H. Jones, S. Ley, a Aitken, Isoforms of 14-3-3 protein can form homo- and heterodimers in vivo and in vitro: implications for function as adapter proteins. FEBS Lett. 368, 55-58 (1995).

73. T. Yamauchi, H. Nakata, H. Fujisawa, A new activator protein that activates tryptophan 5-monooxygenase and tyrosine 3-monooxygenase in the presence of $\mathrm{Ca2+-}$, calmodulin dependent protein kinase. Purification and characterization. J. Biol. Chem. 256, 5404-5409 (1981).

74. T. Ichimura, T. Isobe, T. Okuyama, T. Yamauchi, H. Fujisawa, Brain 143-3 protein is an activator protein that activates tryptophan 5- 


\section{References}

monooxygenase and tyrosine 3-monooxygenase in the presence of Ca2+,calmodulin-dependent protein kinase II. FEBS Lett. 219, 79-82 (1987).

75. T. Ichimura et al., Molecular cloning of cDNA coding for brain-specific 14-3-3 protein, a protein kinase-dependent activator of tyrosine and tryptophan hydroxylases. Proc. Natl. Acad. Sci. U. S. A. 85, 7084-7088 (1988).

76. T. Isobe et al., Distinct forms of the protein kinase-dependent activator of tyrosine and tryptophan hydroxylases. J. Mol. Biol. 217, 125-132 (1991).

77. N. Bonnefoy-Bérard et al., Inhibition of phosphatidylinositol 3-kinase activity by association with 14-3-3 proteins in T cells. Proc. Natl. Acad. Sci. U. S. A. 92, 10142-6 (1995).

78. H. Leffers et al., Molecular cloning and expression of the transformation sensitive epithelial marker stratifin. A member of a protein family that has been involved in the protein kinase $\mathrm{C}$ signalling pathway. J. Mol. Biol. 231, 982-98 (1993).

79. A. Aitken, 14-3-3 proteins on the MAP. Trends Biochem. Sci. 20, 95-97 (1995).

80. A. Aitken, D. Jones, Y. Soneji, S. Howell, 14-3-3 Proteins: Biological Function and Domain Structure. Biochem. Soc. Trans. 23, 605-611 (1995).

81. M. Tanji, R. Horwitz, G. Rosenfeld, J. C. Waymire, Activation of protein kinase $C$ by purified bovine brain 14-3-3: comparison with tyrosine hydroxylase activation. J. Neurochem. 63, 1908-1916 (1994).

82. a Toker et al., Multiple isoforms of a protein kinase $\mathrm{C}$ inhibitor (KCIP1/14-3-3) from sheep brain. Amino acid sequence of phosphorylated forms. Eur.J.Biochem. 206, 453-461 (1992).

83. A. Morgan, R. D. Burgoyne, Exo1 and Exo2 proteins stimulate calciumdependent exocytosis in permeabilized adrenal chromaffin cells. Nature. 355, 833-6 (1992).

84. a Morgan, R. D. Burgoyne, Interaction between protein kinase $\mathrm{C}$ and 
Exo1 (14-3-3 protein) and its relevance to exocytosis in permeabilized adrenal chromaffin cells. Biochem. J. 286 ( Pt 3, 807-811 (1992).

85. H. Fu, J. Coburn, R. J. Collier, The eukaryotic host factor that activates exoenzyme $S$ of Pseudomonas aeruginosa is a member of the 14-3-3 protein family. Proc. Natl. Acad. Sci. U. S. A. 90, 2320-2324 (1993).

86. E. Markiewicz, R. Rzepecki, J. Szopa, Molecular cloning and sequencing of the cDNA encoding plant nuclear matrix endonuclease. Acta Biochim. Pol. 41, 137-8 (1994).

87. C. Oecking, C. Eckerskorn, E. W. Weiler, The fusicoccin receptor of plants is a member of the 14-3-3 superfamily of eukaryotic regulatory proteins. FEBS Lett. 352, 163-166 (1994).

88. J. Ford et al., 14-3-3 protein homologs required for the DNA damage checkpoint in fission yeast. Science (80-. ). 265, 533-535 (1994).

89. G. Reuther, H. Fu, L. Cripe, R. Collier, A. Pendergast, Association of the protein kinases c-Bcr and Bcr-Abl with proteins of the 14-3-3 family. Science (80-. ). 266, 129-133 (1994).

90. D. S. Conklin, 14-3-3 Proteins Associate with cdc25 Phosphatases. Proc. Natl. Acad. Sci. 92, 7892-7896 (1995).

91. C. Peng, Mitotic and G2 Checkpoint Control: Regulation of 14-33 Protein Binding by Phosphorylation of Cdc25C on Serine-216. Science (80-. ). 277, 1501-1505 (1997).

92. A. J. Muslin, J. W. Tanner, P. M. Allen, A. S. Shaw, Interaction of 14-3-3 with Signaling Proteins Is Mediated by the Recognition of Phosphoserine. Cell. 84, 889-897 (1996).

93. K. Rittinger et al., Structural analysis of 14-3-3 phosphopeptide complexes identifies a dual role for the nuclear export signal of 14-3-3 in ligand binding. Mol. Cell. 4, 153-166 (1999).

94. J. Zha, H. Harada, E. Yang, J. Jockel, S. J. Korsmeyer, Serine phosphorylation of death agonist BAD in response to survival factor results in binding to 14-3-3 not BCL-X(L). Cell. 87, 619-628 (1996).

95. J. G. Pastorino, M. Tafani, J. L. Farber, Tumor necrosis factor induces phosphorylation and translocation of BAD through a 


\section{References}

phosphatidylinositide-3-OH kinase-dependent pathway. J. Biol. Chem. 274, 19411-19416 (1999).

96. a Kelekar, B. S. Chang, J. E. Harlan, S. W. Fesik, C. B. Thompson, Bad is a $\mathrm{BH} 3$ domain-containing protein that forms an inactivating dimer with Bcl-XL. Mol. Cell. Biol. 17, 7040-6 (1997).

97. X. Yang et al., Structural basis for protein-protein interactions in the 143-3 protein family. Proc. Natl. Acad. Sci. U. S. A. 103, 17237-17242 (2006).

98. B. Wang Yang, H., Liu, Y.S., Jelinek, T., Zhang, L., Ruoslahti, E., Isolation of high-affinity peptide antagonist of 14-3-3 proteins by phage display. Biochemistry. 12499-504, 12499-12504 (1999).

99. D. K. Morrison, The 14-3-3 proteins: integrators of diverse signaling cues that impact cell fate and cancer development. Trends Cell Biol. 19, 16-23 (2009).

100. M. Chaudhri, M. Scarabel, A. Aitken, Mammalian and yeast 14-3-3 isoforms form distinct patterns of dimers in vivo. Biochem. Biophys. Res. Commun. 300, 679-685 (2003).

101. Y. M. Gu et al., Protein kinase A phosphorylates and regulates dimerization of 14-3-3ろ. FEBS Lett. 580, 305-310 (2006).

102. J. M. Woodcock, J. Murphy, F. C. Stomski, M. C. Berndt, A. F. Lopez, The dimeric versus monomeric status of $14-3-3 \zeta$ is controlled by phosphorylation of Ser58 at the dimer interface. J. Biol. Chem. 278, 36323-36327 (2003).

103. D. Liu et al., Crystal structure of the zeta isoform of the 14-3-3 protein. Nature. 376, 191-4 (1995).

104. B. Xiao et al., Structure of a 14-3-3 protein and implications for coordination of multiple signalling pathways. Nature. 376 (1995), pp. $188-191$.

105. M. B. Yaffe, How do 14-3-3 proteins work? - Gatekeeper phosphorylation and the molecular anvil hypothesis. FEBS Lett. 513, 53-57 (2002).

106. A. K. Gardino, S. J. Smerdon, M. B. Yaffe, Structural determinants of 
14-3-3 binding specificities and regulation of subcellular localization of 14-3-3-ligand complexes: A comparison of the X-ray crystal structures of all human 14-3-3 isoforms. Semin. Cancer Biol. 16, 173-182 (2006).

107. C. Anders et al., A semisynthetic fusicoccane stabilizes a proteinprotein interaction and enhances the expression of $\mathrm{K}+$ channels at the cell surface. Chem. Biol. 20, 583-93 (2013).

108. H. Yuan, K. Michelsen, B. Schwappach, 14-3-3 dimers probe the assembly status of multimeric membrane proteins. Curr. Biol. 13, 63846 (2003).

109. M. J. Knape et al., Divalent Metal lons $\mathrm{Mg}^{2+}$ and $\mathrm{Ca}^{2+}$ Have Distinct Effects on Protein Kinase A Activity and Regulation. ACS Chem. Biol. 2, 150805081002004 (2015).

110. D. Moll et al., Biomolecular interaction analysis in functional proteomics. J. Neural Transm. 113, 1015-32 (2006).

111. K. Muda et al., Parkinson-related LRRK2 mutation R1441C/G/H impairs PKA phosphorylation of LRRK2 and disrupts its interaction with 14-3-3. Proc. Natl. Acad. Sci. U. S. A. 111, E34-E43 (2014).

112. T. Sandmann, J. M. Herrmann, J. Dengjel, H. Schwarz, A. Spang, Suppression of coatomer mutants by a new protein family with COPI and COPII binding motifs in Saccharomyces cerevisiae. Mol. Biol. Cell. 14, 3097-113 (2003).

113. C. Girard et al., p11, an annexin II subunit, an auxiliary protein associated with the background $\mathrm{K}+$ channel, TASK-1. EMBO J. 21, 4439-4448 (2002).

114. O. Nufer, H.-P. Hauri, ER export: call 14-3-3. Curr. Biol. 13, R391-3 (2003).

115. L. H. Boyle, A. K. Gillingham, S. Munro, J. Trowsdale, Selective export of HLA-F by its cytoplasmic tail. J. Immunol. 176, 6464-6472 (2006).

116. B. Coblitz, M. Wu, S. Shikano, M. Li, C-terminal binding: An expanded repertoire and function of 14-3-3 proteins. FEBS Lett. 580, 1531-1535 (2006).

117. A. Kagan et al., 14-3-3 amplifies and prolongs adrenergic stimulation of 


\section{References}

HERG K + channel activity. EMBO J. 21, 1889-1898 (2002).

118. C. G. Nichols, KATP channels as molecular sensors of cellular metabolism. Nature. 440, 470-476 (2006).

119. N. Zerangue, B. Schwappach, Y. N. Jan, L. Y. Jan, A new ER trafficking signal regulates the subunit stoichiometry of plasma membrane K(ATP) channels. Neuron. 22, 537-548 (1999).

120. K. Heusser et al., Scavenging of 14-3-3 proteins reveals their involvement in the cell-surface transport of ATP-sensitive $\mathrm{K}+$ channels. J. Cell Sci. 119, 4353-4363 (2006).

121. T. Kuwana, P. a Peterson, L. Karlsson, Exit of major histocompatibility complex class II-invariant chain p35 complexes from the endoplasmic reticulum is modulated by phosphorylation. Proc. Natl. Acad. Sci. U. S. A. 95, 1056-61 (1998).

122. K. Michelsen, H. Yuan, B. Schwappach, Hide and run. Arginine-based endoplasmic-reticulum-sorting motifs in the assembly of heteromultimeric membrane proteins. EMBO Rep. 6, 717-722 (2005).

123. S. Shikano, M. Li, Membrane receptor trafficking: evidence of proximal and distal zones conferred by two independent endoplasmic reticulum localization signals. Proc. Natl. Acad. Sci. U. S. A. 100, 5783-5788 (2003).

124. P. W. Hoffman, A. Ravindran, R. L. Huganir, Role of phosphorylation in desensitization of acetylcholine receptors expressed in Xenopus oocytes. J Neurosci. 14, 4185-4195 (1994).

125. J. B. Shabb, Physiological substrates of cAMP-dependent protein kinase. Chem. Rev. 101, 2381-411 (2001). 


\section{Curriculum vitae}

Personal details:

Name and Surname:

Markus Kilisch

Address:

Im Hassel 13

37077 Göttingen

Birth date:

05.08.1984

Birthplace:

Osterode (Harz)

Nationality:

German

\section{Education:}

2004-2010 Studies at the Faculty of chemistry, Georg-AugustUniversity Göttingen

Research work for my diploma thesis in the workgroup of Professor Dr. Claudia Steinem

Title: Impedanzspektroskopische Charakterisierung festkörperunterstützter Membranen in Gegenwart des Transmembranproteins hVDAC1

2011

Graduation from the faculty of chemistry

$2012-2016$

Research work for my PhD thesis in the workgroup of Prof. Dr. Blanche Schwappach at the Institute of Molecular Biology, University Medical School (UMG), Georg-August-University Göttingen 


\section{Publication list:}

Research topic related publications:

2015

Kilisch, M., Lytovchenko, O., Schwappach, B., Renigunta, V., and Daut, J. (2015). The role of proteinprotein interactions in the intracellular traffic of the potassium channels TASK-1 and TASK-3. Pflügers Arch. - Eur. J. Physiol. 467, 1105-1120.

2016

Kilisch, M., Lytovchenko, O., Arakel, E.C., Bertinetti, D., and Schwappach, B. (2016). A dual phospho-rylation switch controls 14-3-3-dependent cell surface expression of TASK-1. J. Cell Sci. 129, 831-842.

Method related publications:

2013

Lytovchenko, O., Melin, J., Schulz, C., Kilisch, M., Hutu, D.P., and Rehling, P. (2013). Signal recognition initiates reorganization of the presequence translocase during protein import. EMBO J. 32, 886-898.

Pilla, E., Kilisch, M., Lenz, C., Urlaub, H., and GeissFriedlander, R. (2013). The SUMO1-E67 interacting loop peptide is an allosteric inhibitor of the dipeptidyl peptidases 8 and 9. J. Biol. Chem. 288, 32787-32796. Gomkale, R., Schendzielorz, A., Schmidt, B., Liepold, T., Ficner, R., Jahn, O., et al. (2015). A presequencebinding groove in Tom70 supports import of Mdl1 into mitochondria. Biochim. Biophys. Acta - Mol. Cell Res. 1853, 1850-1859. 
Ilgen, P., Grotjohann, T., Jans, D.C., Kilisch, M., Hell, S.W., and Jakobs, S. (2015). RESOLFT Nanoscopy of Fixed Cells Using a Z-Domain Based Fusion Protein for Labelling. PLoS One 10, e0136233.

Isernhagen, A., Malzahn, D., Viktorova, E., Elsner, L., Monecke, S., von Bonin, F., Kilisch, M., Wermuth, J.M., Walther, N., Balavarca, Y., et al. (2015). The MICA-129 dimorphism affects NKG2D signaling and outcome of hematopoietic stem cell transplantation. EMBO Mol. Med. 7, e201505246.

Göttingen, 2016 